\title{
Urinary incontinence in women : long-term adherence to and outcome of pelvic floor muscle exercise therapy
}

Citation for published version (APA):

Alewijnse, D. (2002). Urinary incontinence in women : long-term adherence to and outcome of pelvic floor muscle exercise therapy. [Doctoral Thesis, Maastricht University]. Universiteit Maastricht. https://doi.org/10.26481/dis.20020308da

Document status and date:

Published: 01/01/2002

DOI:

10.26481/dis.20020308da

Document Version:

Publisher's PDF, also known as Version of record

Please check the document version of this publication:

- A submitted manuscript is the version of the article upon submission and before peer-review. There can be important differences between the submitted version and the official published version of record.

People interested in the research are advised to contact the author for the final version of the publication, or visit the DOI to the publisher's website.

- The final author version and the galley proof are versions of the publication after peer review.

- The final published version features the final layout of the paper including the volume, issue and page numbers.

Link to publication

\footnotetext{
General rights rights.

- You may freely distribute the URL identifying the publication in the public portal. please follow below link for the End User Agreement:

www.umlib.nl/taverne-license

Take down policy

If you believe that this document breaches copyright please contact us at:

repository@maastrichtuniversity.nl

providing details and we will investigate your claim.
}

Copyright and moral rights for the publications made accessible in the public portal are retained by the authors and/or other copyright owners and it is a condition of accessing publications that users recognise and abide by the legal requirements associated with these

- Users may download and print one copy of any publication from the public portal for the purpose of private study or research.

- You may not further distribute the material or use it for any profit-making activity or commercial gain

If the publication is distributed under the terms of Article $25 \mathrm{fa}$ of the Dutch Copyright Act, indicated by the "Taverne" license above, 


\section{URINARY INCONTINENCE IN WOMEN}

Long-term adherence to and outcome of pelvic floor muscle exercise therapy 



\section{URINARY INCONTINENCE IN WOMEN}

\section{Long-term adherence to and outcome of pelvic floor muscle exercise therapy}

\section{Proefschrift}

ter verkrijging van de graad van doctor aan de Universiteit Maastricht, op gezag van de Rector Magnificus, Prof. dr. A.C. Nieuwenhuijzen Kruseman, volgens het besluit van het College van Decanen, in het openbaar te verdedigen op vrijdag 8 maart 2002 om 12.00 uur

door

Dianne Alewijnse 


\section{Promotor}

Prof. dr. Bart van den Borne

\section{Co-promotores \\ Dr. Ilse Mesters \\ Dr. Job Metsemakers}

\section{Beoordelingscommissie}

Prof. dr. Nanne de Vries (voorzitter)

Prof. dr. Philip van Kerrebroeck

Prof. dr. Toine Lagro-Janssen (Katholieke Universiteit Nijmegen)

Prof. dr. Maaike Meijer

Prof. dr. Onno van Schayck

\section{Colofon}

Omslag: Erica van Broeckhuijsen, Utrecht (beeld); Unigraphic, Maastricht (design) Druk: Unigraphic, Maastricht

ISBN 90-5681-123-1

The study presented in this thesis was conducted under the auspices of HEALTH (The Maastricht Health Research Institute for Prevention and Care/ Department of Health Education and Health Promotion)/ EXTRA (Research Institute for Extramural and Transmural Health Care/ Department of General Practice). HEALTH/EXTRA is part of the Netherlands School of Primary Care Research $(\mathrm{CaRe})$, which has been acknowledged since 1995 by the Royal Dutch Academy of Art and Sciences (KNAW). The research was funded by a grant (28-2505) from the Netherlands Care Research (ZON/MW). 


\section{CONTENTS}

Chapter 1 General introduction

Chapter 2 Program development for promoting adherence during and after exercise therapy for urinary incontinence

Chapter 3 Predictors of intention to adhere to physiotherapy among women with urinary incontinence

Chapter 4 Predictors of long-term adherence to pelvic floor muscle exercise therapy among women with urinary incontinence

Chapter 5 Effectiveness of pelvic floor muscle exercise therapy supplemented with a health education program to promote long-term adherence among women with urinary incontinence

Chapter 6 General discussion

Summary

Samenvatting

Appendix: Sex-Specific health education

References

Dankwoord 
In memory of Marij Ebeling 


\section{CHAPTER 1}

\section{General introduction}

This thesis describes the development, implementation and evaluation of a specific health education program for women with urinary incontinence (UI). The goal of the program was to promote long-term adherence to and therapy outcome of individual physiotherapeutic Pelvic Floor Muscle Exercise (PFME) therapy, a conservative treatment for UI. This research project was inspired by three important insights. First, the growing body of evidence that PFME therapy is effective for women with UI but that long-term success is hampered by non-adherence. Second, the insight that health education can be an important means to promote adherence to physiotherapeutic exercise therapy. And third, that targeting health education to individual needs can enhance its effectiveness. Important questions for research were: What are significant predictors of long-term adherence to PFME therapy among women with UI? And, based on the assumption that better adherence behavior leads to better therapy outcomes: Does PFME therapy supplemented with a specific health education program result in better long-term adherence behavior and better therapy outcomes compared to PFME therapy alone?

A systematic and planned approach was applied for the development, implementation and evaluation of the health education program (PRECEDE, Green and Kreuter, 1991 and 1999; Intervention Mapping, Bartholomew et al., 2001). The planning phase started with a study of the health problem UI, the health behavior adherence to PFME therapy and the determinants of this adherence behavior. Subsequently, theory-driven behavioral change methods based on social cognition theory were used for the development of the program that was to be supplemented to physiotherapeutic PFME therapy. Then, the program was implemented and evaluated in a longitudinal randomized controlled trial.

This introduction provides a brief overview of the design of the study: the planning phase, the development of the health education program and the evaluation. Further, an outline of the content of the thesis is given. 


\section{Analysis of the health problem of urinary incontinence}

UI is a prevailing health problem affecting many community-dwelling adults, of whom $75 \%$ are women. Overall, the estimated prevalence of UI among adult ( $>18$ years)

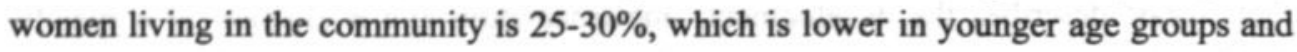
higher in older age groups (Hannestad et al., 2000; Holst and Wilson, 1988; Jolleys, 1988; Rekers et al., 1992; Thomas et al., 1980). The prevalence of UI not only increases with age, but also varies with the definition of incontinence, measures used to quantify urine leakage and with the population under study (selected or open population).

The three most prevalent types are stress, urge and mixed UI (Jolleys, 1988; LagroJanssen, 1991). Stress incontinence is defined as the complaint of involuntary loss of urine during for instance coughing, sneezing and physical exertion such as sport activities and sudden changes of position. Urge incontinence is the complaint of the involuntary loss of urine associated with a sudden, strong desire to void (urgency) (Abrams et al., 1988; Blaivas et al., 1997). Urge incontinence, as well as symptoms of urgency and frequency ( $>7$ voidings per day), are typical symptoms of an overactive bladder (Messelink et al., 1999 and 2000). In mixed UI symptoms of both stress and urge incontinence are present (Blaivas et al., 1997). Of the women with these types of UI, approximately $50 \%$ has symptoms of stress, $10 \%$ of urge and $30 \%$ of mixed UI (Hannestad et al., 2000; Van der Vaart et al., 2000). The high prevalence rates of UI bring forward an enormous financial impact on the health care costs of countries. For example, in the Netherlands, up to 80 million euros are spent yearly on incontinence pads alone, those used in residential homes not taken into account (Health Council, 2001; Janssen and Miltenburg, 1998).

UI is associated with reduced physical, social and mental well being in terms of, among others, embarrassment, fear of offensive odour, refraining from social, physical and sexual activities and loss of self esteem (Berghmans et al., 1998a; Berghmans et al., 2000; Dugan et al., 2000; Wyman et al., 1990). However, it is also known that many women report that the symptoms do not bother them so much, and this is one of the main reasons that more than half of the women with incontinence symptoms don't seek help (Lagro-Janssen et al., 1990; Rekers et al., 1992). Related reasons for not seeking help are: symptoms are not considered to be a serious medical condition, a lack of awareness of treatment options, fear of surgery, shame and embarrassment (Dugan et al., 2001; Lagro-Janssen et al., 1990; Shaw et al., 2001). Furthermore, inadequate health education as well as consistent and aggressive gendered 
commercials of incontinence pads in the media contribute to already persistent lay beliefs that UI is a normal women's complaint after childbirth or with increasing age (Janetzky, 1993). In addition, most women have hardly any knowledge about incontinence and are not aware of the fact that symptoms get worse when not treated (Cammu et al., 1991; Janetzsky, 1993; Nygaard et al., 1990; O’Dowd, 1993).

\section{Treatment for urinary incontinence: Pelvic floor muscle exercise therapy}

Nowadays, PFME therapy is recommended as first-choice treatment for women with either stress, urge or mixed UI, when other physical impairments causing UI have been excluded and possible urinary tract infections have been treated successfully. In 1948, Kegel first reported about the benefits of pelvic floor muscle exercises (PFMEs) for women with these types of UI (Kegel, 1948). Nevertheless, surgery soon became the first-choice treatment for stress incontinence. Costs of and morbidity after surgery, together with a growing awareness that evidence for its effectiveness is weak, led to a renewed interest in the conservative PFME therapy for stress incontinence in the early 1980s (Black and Downs, 1996; Bø et al., 2000a). In The Netherlands, the pre- and post natal gymnastics have influenced the renewed interest in PFME therapy as well. The main goal of PFME therapy is making the patient aware of the functions of the body, especially of the pelvic floor muscles in relation to breathing, body posture and movement, through patient education and exercise therapy to reduce impairments and to restore functions and participation (Versprille-Fischer, 1995).

Many studies on the effectiveness of PFME therapy for women with stress incontinence have been published since the early 1980 's. At the start of this research project in 1996, three reviews and one meta-analysis on the effectiveness of PFME therapy for women with stress incontinence had been published (Bø, 1995a; Kruif and Van Wegen, 1996; Mouritsen, 1994; Wells, 1990). By that time, the idea was that PFME therapy was effective in women with symptoms of stress incontinence compared to no therapy (Burns et al., 1993; Lagro-Janssen et al., 1992; O'Brien et al., 1991). Therapy for urge incontinence most often included pharmacological treatment or behavioral treatment with bladder training (Andersson et al., 1999; Bø and Berghmans, 2000b; Burgio et al., 2000). In the last decade, good results have also been reported in women with mixed or urge incontinence who were treated with PFME therapy combined with bladder training. The rationale is that unstable bladder 
contractions can be inhibited by a contraction of the pelvic floor muscles (Flynn et al., 1994; Mouritsen et al., 1991; Nygaard et al., 1996; Wyman et al., 1998).

As the project proceeded, three systematical reviews were being produced by several members of the Cochrane Incontinence Review Group of the Cochrane Collaboration (Berghmans et al., 1998a; Berghmans et al., 2000; Hay-Smith et al., 2001). These reviews revealed that there is sufficient evidence that PFME therapy is effective for women with either stress or mixed UI, and that favorable results for urge incontinence could be expected as well. The reviewers also concluded that comparison of the effectiveness of PFME therapy is very difficult and calculating effect rates in metaanalyses is hampered because of the inconsistent use of outcome variables, and weak methodological quality of studies. Another problem hampering comparison of study results is the wide variability in PFME therapy programs that are guided by either general practitioners (GPs), specialized nurses or physiotherapists, intensively guided or conducted as home-programs, given as individual treatment or in groups.

Most studies present effect rates as combined cure and symptom improvement rates, mostly counting those women improved by $50 \%$ or more on the primary outcome measure of effect. Short-term effect rates may exceed $70 \%$, while long-term effect rates may reach $50 \%$ when adherence is maintained (Mouritsen, 1994; Mouritsen and Schiøtz, 2000). Many authors found that symptoms returned or increased again after therapy had ended and concluded that declining adherence levels were probably the most important cause for declining long-term effect rates (Bø and Talseth, 1996; Cammu and Van Nylen, 1994; Chen et al., 1999; Hahn et al., 1993; Lagro-Janssen and Van Weel, 1998; Ramsey and Thou, 1990). Consequently, many researchers expressed the need for further studies into adherence behavior to PFME therapy. Important questions are: what are determinants of adherence to PFME therapy and how can this particular adherence be promoted?

\section{The health behavior: adherence to PFME therapy and its determinants}

Non-compliance or non-adherence is one of the most important problems of health care. Adherence is defined as the ability or willingness of a patient to follow health advice that are brought about in collaboration between a health professional and a patient. Thus, the patient plays an active role in the health care plan. In contrast, compliance is defined as the extent to which a patient's behavior coincides with medical or health advice. This suggests that the patient has a passive role and confirms 
a request made by the health professional (DiMatteo et al., 1994; Meichenbaum and Turk, 1987; Van Campen and Sluijs, 1989). The term adherence applies best to physiotherapeutic exercise therapy as this therapy is mainly directed at behavioral change and requires active patient involvement. Adherence behavior is often not a matter of all or nothing but has to be regarded as a continuum and patients may have many reasons why they adhere completely, partly or not at all (Sluijs et al., 1993; Steiner and Earnest, 2000).

Information about adherence to PFME therapy is limited. Several studies found that two to seven years after organised training 15 to $70 \%$ of the women studied maintained doing pelvic floor muscle exercises at least once a week or more (Be and Talseth, 1996; Cammu and Van Nylen, 1994; Hahn et al., 1993; Lagro-Janssen and Van Weel, 1998; Ramsey and Thou, 1990). However, prescribed exercise regimens differed among these studies which hinder clear comparison of adherence rates. The best results and adherence behavior were found in women who had intensive training programs guided by motivated physiotherapists as compared to women who were shortly instructed at the clinic and further left to train at home and alone (Bø et al., 1990; Cammu and Van Nylen, 1994; Wyman et al., 1998). Three studies found that adherence, both during the period of therapy (including practicing at home) and thereafter, was a significant predictor of long-term success (Bø and Talseth, 1996; Chen et al., 1999; Lagro-Janssen and Van Weel, 1998). Despite efforts to use adherence-promoting strategies such as exercise tapes, reminder phone calls or frequent supervision (Burns et al., 1993; Nygaard et al., 1996), no study really tried to improve adherence behavior with a specific intervention focused on behavior.

The needs assessment for this study consisted of an extensive literature study and interviews with the target population to reveal information about determinants of adherence to PFME therapy. Publications on the effectiveness of PFME therapy suggested many determinants but provided no empirical evidence. Additional and more detailed information was revealed from the interviews and empirical evidence on determinants of adherence was obtained from the few studies available on adherence behavior in general physiotherapeutic exercise therapy. Much of current knowledge about adherence in physiotherapy stems from Dutch research that brought insight into which exercises, instructions and advice physiotherapists prescribe; what problems physiotherapists encounter when educating patients; which determinants are related to patients' adherence to physiotherapy; which theories are useful to enhance 
understanding and improvement of long-term adherence to physiotherapy; and, which strategies can be used to enhance adherence that are applicable in the physiotherapeutic practice (Sluijs et al., 1998). Three important findings are illustrated here.

First, although the promotion of adherence behavior is considered an integral part of patient education concerning physiotherapy, it appeared that patient education in physiotherapy, and especially the part of encouraging adherence behavior, lacks a systematic and theoretically funded behavioral approach (Knibbe and Wams, 1994; Sluijs et al., 1993). Such an approach is expected to enhance the effectiveness of education (Green and Kreuter, 1991). The second finding was that there is a difference between short-term supervised adherence, which is the adherence during the period of therapy sessions, and long-term non-supervised adherence, which is the adherence during the period after the therapy sessions have ended and the patient has to train at home and alone. Little is known about long-term non-supervised adherence in physical therapy, which is excepted to be totally different in nature from short-term supervised adherence and far more difficult to attain. Physiotherapists estimate that $64 \%$ of the patients adhere to exercise regimens and health advice on the short-term but that only $23 \%$ do so on the long-term (Sluijs and Knibbe, 1991).

Third, it was found that short- and long-term adherence were partly related to different determinants. Short-term adherence was found to be negatively related to the barriers that patients perceive (forgetting to do the exercises, difficulties with integrating exercising in dialy life, lack of time, lack of motivation), and positively to feelings of competence and self-efficacy, a positive attitude towards adherence behavior and positive feedback. And, the worse patients perceived their symptoms, the better their adherence during therapy (Knibbe and Wams, 1994; Sluijs, 1991). Important determinants for long-term non-supervised adherence were social norms, the motivation to comply as a result of the perceived social norms, the attitude in terms of outcome expectations of adherence behavior, self-efficacy expectations (Knibbe and Wams, 1994; Kok and Bouter, 1990) and patients' representations of the illness and the concurrent emotions and self-care strategies (eg. drinking less and frequent voiding, see Johnson et al., 2000) (Sluijs et al., 1993). Furthermore, targeting health education was also identified as an important determinant of adherence behavior (Kreuter et al., 2000; Sluijs and Knibbe, 1991). This involves tuning the health education messages to the target group involved, so that the information given closely links up with the perceptions, norms, values and expectations of the target group. Thus, adequate 
instructions and advice that take into account womens' lifestyle and daily life situation are necessary to enable designing of a program in which women can find appropriate answers to their own changing information needs.

In sum, health education to promote adherence behavior needs a systematic and theory-driven approach, educational messages should focus on both determinants of short- and long-term adherence and the information should be targeted at the group of interest.

Much of the determinants found in literature on PFME therapy were also found in literature on general physiotherapy and came up in the interviews. It was further noticed that knowledge, motivation and sex-specific aspects have an important influence on these determinants. To categorize and conceptualize the variables that were found important in determining adherence behavior in a theoretical framework, a social cognition model was used called the Attitude-Social influence-self-Efficacy (ASE) model (De Vries et al., 1988). According to the ASE model, behavioral change is best predicted by someone's intention to perform that behavior. The model assumes that behavioral intention is determined by three types of cognitive proximal factors: attitudes, social influences and self-efficacy expectations. Distal variables such as socio-demographic, psycho-social and medical variables are expected to influence behavioral intention through the proximal variables. Barriers and skills play a role when actual behavior is performed, and this performance leads to a feedback process that, in turn, influences the three proximal variables (De Vries and Mudde, 1998; Lechner, 1998).

The ASE model has been influenced by both the Theory of Planned Behavior (TPB; Ajzen, 1991), which also includes attitudes and social norms, and Bandura's (1986) concept of self-efficacy, a construct derived from the Social Learning Theory (SLT). The TPB and the ASE model partly overlap with respect to the concepts that are integrated but differ in the way these concepts are measured (De Vries and Mudde, 1998). Besides social norms, the ASE model also includes modeling and social pressure/support as social influences of behavior (De Vries et al., 1988). Figure 1 shows how the ASE model is applied in this study and what determinants have been taken into account in the health education program. 


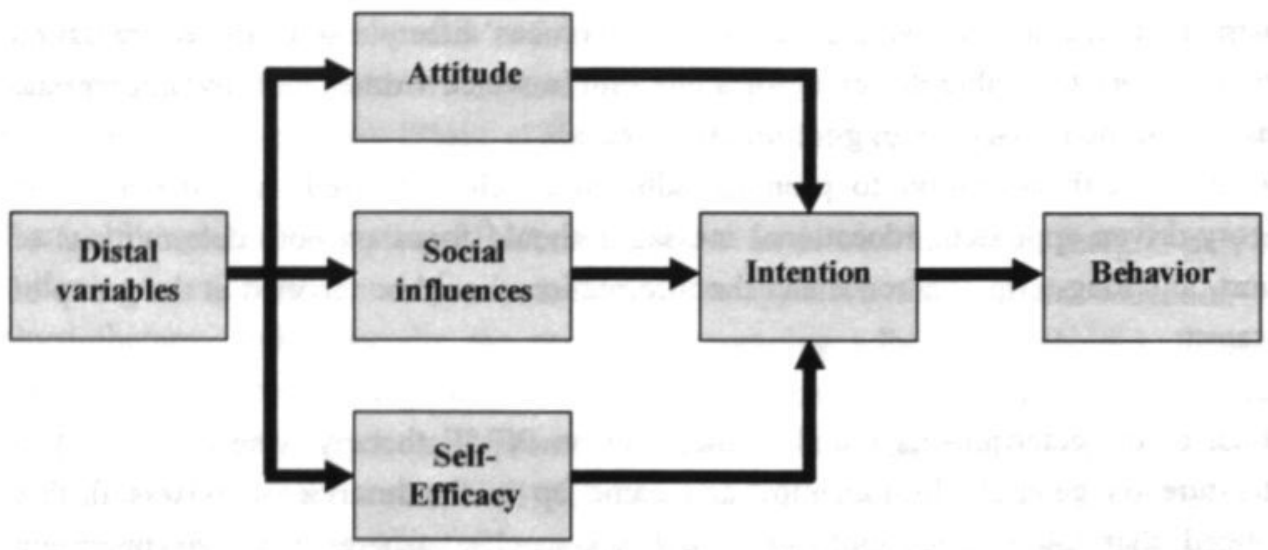

Figure 1.

The ASE model of behavioral determinants (De Vries et al., 1988).

\section{Development of the health education program}

After analyzing the determinants of adherence to PFME therapy, Intervention Mapping was used to further guide the systematic approach of development of the health education program. There was no evidence that some determinants of adherence were more important than others. Therefore, we decided to focus on all identified determinants in the health education program. For each determinant specific aims were formulated and appropriate methods and strategies were selected. For example, for the method of goal setting, the strategy consisted of a filling-in task to write down personal treatment goals, and for the method of modeling, role model stories were the strategy (Bassett and Petrie, 1999; Strecher et al., 1995; see Chapter 2). Furthermore, quality principles for patient education were applied (Mullen et al., 1985). For example, selfevaluation about changes in symptoms was stimulated, and reinforcement was given for positive behavior changes. The information was targeted to women's needs by, among others, implementing and operationalizing criteria for sex-specific health education (see appendix A).

In addition, we developed a minimal, a medium, and a maximum intervention that each followed the physiotherapeutic treatment plan in content (Knibbe and Wams, 1994; Verhulst et al., 1994), to enable investigating what intensity of health education would be necessary to promote adherence effectively. Three health education interventions were developed of which the most important components were 
reminders, guidance of adherence as self-management process, and structured feedback (Cramer, 1995; Leventhal and Cameron, 1987; Leventhal et al., 1998; Prochaska et al., 1992 and 1994):

1) Reminder intervention (Ri): since forgetting was most often mentioned as a barrier for adherence, this intervention consisted of a folder with information about PFME therapy and adherence behavior and several tips to remember adherence behavior. Stickers were included as reminders.

2) Reminder and Self-Help Guide intervention (R-SHGi): a guide (Alewijnse, 1997) addressing facts and myths about UI and pelvic floor muscles, coping with UI, tips to tackle all barriers hampering adherence behavior, and relapse prevention strategies to support the self-management process. The self-help guide also contained the stickers of the $\mathrm{Ri}$ and reminder tips.

3) Reminder, Self-Help Guide and Counseling intervention (R-SHG-Ci): identical to the R-SHGi, with the addition of a counseling scheme for physiotherapists, guiding structural oral feedback and reinforcement to promote adherence behavior.

\section{Research questions and the study design}

The study addressed the following research questions:

1. Which are the relevant predictors of intention to adhere to PFME therapy at the onset of therapy?

2. Which are the relevant predictors of long-term adherence to PFME therapy?

3. What is the influence of PFME therapy supplemented with the health education program on long-term adherence behavior and on predictors of adherence?

4. Does PFME therapy supplemented with a health education program result in better therapy outcomes compared to PFME therapy alone, one year after therapy?

5. Does PFME therapy supplemented with a health education program result in better adherence behavior compared to PFME therapy alone, one year after therapy?

It was hypothesized that better adherence behavior is related to better therapy outcomes. The second hypothesis was that the most complete health education program (R-SHG-Ci) would be more effective than the other two programs, as well as 
that the reminder-self-help guide intervention would be more effective than the reminder intervention.

A longitudinal randomized controlled trial with three experimental and one control condition was set up. The control condition consisted of individual physiotherapeutic PFME therapy alone, representing usual care. The experimental conditions consisted of PFME therapy with one of the three health education interventions. The two primary outcome measures were adherence and therapy outcome. Adherence was operationalized as the number of days per week women had followed the behavioral advice of the physiotherapist, and therapy outcome as the weekly frequency of wet episodes (Kerssens et al., 1996; Nygaard and Holcomb, 2000; Wyman et al., 1988).

Participants were recruited from the Registration Network Family Practices (RegistratieNet Huisartspraktijken, RNH) (Metsemakers et al., 1992). Since many women do not seek medical treatment for UI (Shaw et al., 2001), community-dwelling women over 17 years old with at least one of the relevant risk factors, such as vaginal delivery or asthma, were selected from the database of the RNH (Diokno et al., 1990; Milsom et al., 1993; Snooks et al., 1990; Yarnell et al., 1982). GPs were involved in the diagnosis of women with symptoms of UI and the selection of the participants. Inclusion criteria were ability to complete questionnaires, full understanding of the Dutch language and completion of the consent form. Excluded from the study were women without symptoms of stress, urge or mixed UI based on their anamneses, women suffering from neurological conditions such as MS, CVA and spina bifida or suffering from venereal disease, women with viral infections, women using medication for UI or using medication that enhances/influences UI, women who were pregnant or within three months after delivery or women who had been operated upon for UI, and women with physical impairments making PFME therapy impossible.

Physiotherapists specialized in the field of PFME therapy with a practice in the residential area of the participating general practices were recruited. They were trained to use a protocol checklist for PFME therapy that was developed as a standard for all conditions. For the experimental conditions, the use of the health education intervention was indicated in the protocol checklist. The treatment conditions were randomly allocated to the physiotherapists and patients. The evaluation instruments consisted of self-administered questionnaires and diaries that had to be completed by the participants at four moments: before the onset of therapy (pretest), immediately after therapy had ended (posttest), and three- and twelve months later. 


\section{Outline of the thesis}

Chapter 2 holds a detailed description of the development process of the health education program. To check whether the health education program was targeted at the relevant determinants of adherence to PFME therapy, a cross-sectional study on the pretest data was conducted to assess significant predictors of the intention to adhere to PFME therapy, which is described in Chapter 3. The question whether the components of the health education program had been implemented as planned is addressed to in Chapters 4 and 5. Chapter 4 presents the assessment of the significant predictors of long-term adherence behavior one year after therapy, and the evaluation of the influence of the health education program on adherence to PFME therapy and predictors of adherence behavior. The evaluation of the effectiveness of PFME therapy supplemented with the health education program compared to PFME therapy alone, on incontinence symptoms and adherence behavior, is described in Chapter 5. This chapter also provides results from the process evaluation about the use and appreciation of the health education program. Finally, Chapter 6 contains a general discussion in which the main conclusions are presented, methological issues are discussed, future research questions are proposed and implications for practice are suggested. 


\title{
CHAPTER 2
}

\section{Program development for promoting adherence during and after exercise therapy for urinary incontinence}

\begin{abstract}
This paper presents the development process of a health education program to promote adherence to a pelvic floor muscle exercise (PFME) therapy for women with urinary incontinence. The development process started with a needs assessment phase in which the health problem, health-related quality of life, and behavioral and environmental determinants were assessed. Guided by the Intervention Mapping (IM) approach, program objectives were formulated and, on the basis of both empirical and theoretical data, intervention methods for influencing determinants of adherence to PFME therapy were chosen and translated into practical strategies. This information was assimilated to a transparent description of the program design. The theoretical rationale of the program was based on the Transtheoretical Model, the Self-Regulation Theory and principles of targeted communication and sex-specific health care.
\end{abstract}

Manuscript accepted for publication as: Dianne Alewijnse, Ilse E.P.E. Mesters, Job F.M. Metsemakers, Bart H.W. van den Borne. Program development for promoting adherence during and after exercise therapy for urinary incontinence. Patient Education and Counseling. 


\section{INTRODUCTION}

There is a paucity of transparent and detailed papers on the planning and development processes of health education programs that include both a rationale for the selection of the theory and its concepts, and an account of their translation into practice (Bartholomew et al., 2000b; Cullen et al., 1998; Green, 2000; Kok et al., 2000; Mullen et al., 1985; Nutbeam, 1996). However, conducting valid and reliable research requires a transparent description of materials and methods used. Applying this rule to health education research implies systematic, transparent and detailed descriptions of the planning and development processes of health education programs so that, in terms of diffusion, the application and replication of effective programs by others is facilitated (Green, 2000).

The purpose of this paper is therefore to give a transparent description of the development process of a health education program to promote adherence to a physiotherapeutic pelvic floor muscle exercise (PFME) therapy for women with urinary incontinence (UI). A detailed account of the program components is accomplished by combining the description of the theoretical methods and their operationalization in health education strategies. The Intervention Mapping (IM) approach (Bartholomew et al., 2001) was used to systematically guide the process of program development. Figure 1 shows the steps within the IM process, including the preceding process of needs assessment (Green and Kreuter, 1999). In short, this process boils down to the following when applied to UI and PFME therapy.

During the needs assessment, we collected epidemiological information about the health problem UI and its effects on quality of life. PFME therapy was found to be an effective treatment. However, its effectiveness seemed highly dependent on adherence to all behavioral aspects of the treatment and many studies in this field expressed the need for further improvement of adherence to PFME therapy. Therefore, adherence to PFME therapy was identified as the health behavior for further investigation and we performed a systematic analysis of the determinants of this behavior. We then completed the first IM step by selecting performance objectives, specifying important and changeable determinants of adherence to PFME therapy, and considering whether to differentiate the target population. Performance objectives and determinants were combined to create a matrix of learning and change objectives. Next, we described the program and its components, combining the second step of selecting intervention methods and translating them into practical strategies, with the third step of designing 
the program. Steps four and five - the implementation and evaluation processes involving a randomized controlled trial - are described in Chapters 4 and 5 .

\begin{tabular}{|l} 
Needs Assessment \\
Identifying population at risk, quality of life and health problem \\
Distinguishing behavioral and environmental \\
causes of health risk behavior \\
Reviewing key determinants
\end{tabular}

Figure 1.

The needs assessment and Intervention Mapping process, derived from (Bartholomew et al., 2001) and adapted for this chapter. 


\section{NEEDS ASSESSMENT}

\section{Analysis of health problems}

UI is defined by the International Continence Society as the involuntary loss of urine which is objectively demonstrable and a social or hygienic problem (Abrams et al., 1988). The three most prevalent types of UI are stress, urge and mixed UI (Jolleys, 1988; Lagro-Janssen, 1991). Stress incontinence is the complaint of involuntary loss of urine during, for instance, coughing, sneezing and physical exertion, such as sport activities and sudden changes of position. Urge incontinence is the complaint of involuntary loss of urine associated with a sudden, strong desire to void. In mixed UI, symptoms of both stress and urge incontinence are present (Abrams et al., 1988; Blaivas et al., 1997).

UI is a common complaint among community-dwelling adults of whom $75 \%$ are women. Overall, the estimated prevalence of UI among adult ( $>18$ years) women

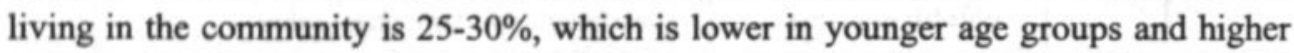
in older age groups (Hannestad et al., 2000; Holst and Wilson, 1988; Jolleys et al., 1988; Rekers et al., 1992; Thomas et al., 1980). The high prevalence rates bring forward an enormous financial impact on the health care costs of countries. For example, in the Netherlands, up to 80 million Euros are spent yearly on incontinence pads alone, those used in residential homes not taken into account (Health Council, 2000; Janssen and Miltenburg, 1998). UI is associated with reduced physical, social and mental wellbeing caused by embarrassment, fear of offensive odor, and loss of self-esteem. Due to the subjective nature of the experience, these impairments may correlate poorly with the objective severity of incontinence symptoms (Dugan et al., 2000; Holtedahl et al., 1998). PFME therapy is recommended as first-choice treatment for women with symptoms of stress, urge and mixed UI when other physical impairments causing UI have been excluded and possible urinary tract infections have been treated successfully (Berghmans et al., 1998a; Berghmans et al., 2000; Bø, 1995a; Lagro-Janssen et al., 1995).

\section{Pelvic floor muscle exercise therapy}

Physiotherapeutic PFME therapy for stress incontinence focuses on the integration of the automatic and subconscious use of the pelvic floor muscles during the daily posture 
and movement pattern, and on the integration of pelvic floor muscle exercises (PFMEs) in daily life. For urge and mixed incontinence, the therapy focuses first on bladder training in which a normal voiding frequency of approximately seven voidings per day is trained. Second, the therapy includes the same program as for stress incontinence with the addition of learning to prevent leakage when feeling an urge, by contracting the pelvic floor muscles to inhibit contraction of the bladder. The therapy includes teaching about the anatomy and function of the bladder, the pelvic floor muscles, the continence mechanism, toilet behavior and education on the importance of adherence to the advice (Berghmans et al., 1998b; Bø et al., 1990; Bø, 1995a; Miller et al., 1998; Mouritsen et al., 1991; Nygaard et al., 1996; Lagro-Janssen et al., 1995; Payne, 2000; Versprille-Fischer, 1995; Wyman et al., 1998).

Today, PFME therapy has been proven effective for women with stress and mixed incontinence (Berghmans et al., 1998a; Bø, 1995a; Hay-Smith et al., 2001). Some studies reported favorable results for urge incontinence as well (Berghmans et al., 2000; Nygaard et al., 1996; Wyman et al., 1998). Overall, in studies up to $70 \%$ of the population was cured or improved after therapy (Berghmans et al., 1998a; Bø, 1995a; Burns et al., 1993; Lagro-Janssen et al., 1992; Nygaard et al., 1996), but success rates declined during follow-up as adherence to PFME therapy recommendations deteriorated. This finding can be illustrated by three studies that found that adherence, both during the period of therapy (including practicing at home) and thereafter, was a significant predictor of long-term success (Bø and Talseth, 1996; Chen et al., 1999; Lagro-Janssen and Van Weel, 1998). In one study, $62 \%$ of the women reported good adherence three months after therapy. This declined to $51 \%$ one year after therapy and to $39 \%$ at five-year follow-up, while in the meantime incontinence symptoms had returned or increased significantly (Lagro-Janssen et al., 1992; Lagro-Janssen and Van Weel, 1998). Chen et al. (1999) showed that during a two-year follow-up, continence success rates declined more in the groups with poor and moderate adherence than in the group that adhered well. Bø and Talseth (1996) found that those who exercised three times per week or more had significantly fewer symptoms than those who exercised less frequently, five years after organized PFME training. Nowadays, there is a broad consensus in PFME therapy research that adherence needs improvement. Therefore, insight is needed into the determinants of adherence to PFME therapy (Lagro-Janssen and Van Weel, 1998; Nygaard et al., 1996; Gallo and Staskin, 1997; Wells, 1990). 


\section{Analysis of determinants of adherence}

Determinants of adherence to PFME therapy were first analyzed in publications on the effectiveness of PFME therapy (Ashworth and Hagan, 1993; Bø et al., 1990; Bø, 1995b; Burns et al., 1993; Cammu et al., 1991; Cammu and van Nylen, 1994; Castleden et al., 1984; Dougherty et al., 1993; Gallo and Staskin, 1997; Hahn et al., 1993; Janetzky, 1993; Lagro-Janssen et al., 1994 and 1995; Lagro-Janssen and Van Weel, 1998; Mantle and Versi, 1991; Mouritsen et al., 1991; Nygaard et al., 1990 and 1996; O'Dowd, 1993; Wall and Davidson, 1992; Wilson et al., 1987; Wyman et al., 1998). These studies suggested many determinants of adherence but provided no empirical evidence. Therefore, a second literature analysis was conducted in the field of physiotherapy, providing empirical evidence of determinants of adherence to physical exercise therapy in general (Knibbe and Wams, 1994; Kok and Bouter, 1990; Sluijs et al., 1993; Sluijs and Knibbe, 1991). Third, to reveal additional and more detailed information about determinants of adherence to PFME therapy, fifteen individual interviews and one group discussion with six women suffering from UI were conducted. About half of these women had experience with PFME therapy. In addition, a group discussion was held with seven female physiotherapists who specialized in the field of pelvic floor dysfunctions (PFME therapists). The determinants of adherence to PFME therapy identified in the literature and interviews are summarized and listed in Table 1.

Four aspects of Table 1 are highlighted here: the concept of motivation, the determinants feedback and reinforcement, the influence of sex-specific aspects and knowledge and barriers.

To promote adherence to PFME therapy involves stimulating a behavioral change process in terms of integrating new behaviors in daily life and refraining from behaviors that negatively affect incontinence symptoms. Previous studies have emphasized the importance of positive motivation for adherence to PFME therapy (Cammu et al., 1991; Lagro-Janssen and Van Weel, 1998; Mantle and Versi, 1991) or physiotherapeutic exercise therapy (Kok and Bouter, 1990; Sluijs and Knibbe, 1991). The Transtheoretical model explains behavioral change processes in terms of five motivational stages and is appropriate to many health behaviors (Prochaska et al., 1992 and 1994), including adherence (Willey et al., 2000). In the precontemplation stage, people do not intend to change certain behaviors or adopt new behaviors. In the contemplation stage, people are considering a change, whereas in the preparation stage they make active plans to change and in the action stage they have changed but their 
Table 1. Determinants of adherence to PFME therapy from three sources: literature on PFME therapy (PFME) and general physical exercise therapy (phys.ex), and interviews per motivational stage.

\begin{tabular}{|c|c|c|c|c|}
\hline $\begin{array}{l}\text { Motivational } \\
\text { stage }\end{array}$ & determinants & PFME & phys. ex & Interviews* \\
\hline All stages & $\begin{array}{l}\text { knowledge, barriers } \\
\text { sex-specific aspects }\end{array}$ & {$[29,36,39,48]$} & {$[50-52]$} & $\bar{W}$ \\
\hline Precontemplation & $\begin{array}{l}\text { beliefs, self-care } \\
\text { strategies } \\
\text { illness representation }\end{array}$ & $\begin{array}{l}{[37,44,45,47,48]} \\
{[48]}\end{array}$ & $\begin{array}{l}{[50,52]} \\
{[50-52]}\end{array}$ & w \\
\hline $\begin{array}{l}\text { Contemplation \& } \\
\text { Preparation }\end{array}$ & $\begin{array}{l}\text { perceived norms, values } \\
\text { risk perception } \\
\text { attitude, } \\
\text { outcome expectations, } \\
\text { prognosis } \\
\text { perceived severity of } \\
\text { symptoms }\end{array}$ & $\begin{array}{l}{[37,48]} \\
{[37,48]} \\
{[21,38]} \\
{[12,48]} \\
{[33,37,40]}\end{array}$ & $\begin{array}{l}{[53]} \\
{[53]} \\
{[50,53]} \\
{[51-53]} \\
{[50]} \\
{[50-53]}\end{array}$ & $\begin{array}{l}\text { W, Ph } \\
\text { W, Ph } \\
\text { W, Ph } \\
\text { w } \\
\text { W }\end{array}$ \\
\hline $\begin{array}{l}\text { Action \& } \\
\text { Maintenance }\end{array}$ & $\begin{array}{l}\text { skills, self-efficacy, } \\
\text { clear instructions } \\
\text { cue to action } \\
\text { specific barriers: } \\
\text { - forgetting } \\
\text { - discipline } \\
\text { - time \& energy } \\
\text { - stressful situations } \\
\text { - associations with sex } \\
\text { - unconscious bodily } \\
\text { area } \\
\text { - integrating exercises in } \\
\text { daily life } \\
\text { - fluctuations effectivity } \\
\text { - muscle pain (bladder) } \\
\text { internal feedback: } \\
\text { - progress, social } \\
\text { support } \\
\text { - quality of life, self- } \\
\text { esteem, body esteem } \\
\text { external feedback, } \\
\text { reinforcement, social } \\
\text { support } \\
\text { modeling }\end{array}$ & $\begin{array}{l}{[27]} \\
{[20,21,33,35,49]} \\
{[37]} \\
{[21,37,38,48]} \\
{[37,38]} \\
{[37]} \\
{[37,44]} \\
{[44,48]} \\
{[37]} \\
{[37]} \\
{[12]} \\
{[12,21,24,37,38,39,} \\
41,43] \\
{[39,48]} \\
{[37,39]} \\
{[21,24,27,38,44,46]}\end{array}$ & $\begin{array}{l}{[50-52]} \\
{[53]} \\
\\
{[50,51,53]} \\
{[50-52]} \\
{[50-52]} \\
{[50-52]}\end{array}$ & $\begin{array}{l}\text { w, Ph } \\
\text { w } \\
\text { w, Ph } \\
\text { w, Ph } \\
\text { W, Ph } \\
\text { W } \\
\text { W, Ph } \\
\text { w } \\
\text { w } \\
\text { W, Ph } \\
\text { Ph }\end{array}$ \\
\hline
\end{tabular}

* $\mathrm{W}=$ group and individual interviews with women; $\mathrm{Ph}=$ group interview with PFME therapists 12: Lagro-Janssen et al., 1995; 20: Wyman et al., 1998; 21 : Nygaard et al., 1996; 24: Mouritsen et al., 1991; 27: Bø et al., 1990; 29: Lagro-Janssen and Van Weel, 1998; 33: Burns et al., 1993; 35: Gallo and Staskin, 1997; 36: Mantle and Versi, 1991; 37: Lagro-Janssen et al., 1994; 38: Hahn et al., 1993; 39: Cammu et al., 1991; 40: Cammu and Van Nylen, 1994; 41: Bø, 1995a; 43: Castleden et al., 1984; 44: Janetzky, 1993; 45: O'Dowd, 1993; 46: Wilson et al., 1987; 47: Nygaard et al., 1990; 48: Ashworth and Hagan, 1993; 49: Dougherty et al., 1993; 50: Sluijs et al., 1993; 51: Sluijs and Knibbe, 1991; 52: Kok and Bouter, 1990; 53: Knibbe and Wams, 1994. 
behavior has not yet become a routine. People have integrated the altered behavior in their lives and are trying to sustain it in the maintenance stage. Each motivational stage, or a combination of stages is characterized by several behavioral determinants (see Table 1). A detailed description of program objectives in relation to determinants and of the role of determinants in relation to motivational stages in the program is presented in paragraphs 3.1.4. and 3.2.1. and Tables 2 and 3.

As relapse is inherent to behavioral change, it may occur in any of the motivational stages (Prochaska et al., 1992). Motivation is thus seen as a state of readiness or eagerness to change which may fluctuate from one time or situation to another (Miller and Rollnick, 1991), implying that a person can go back (relapse) and forth between the stages as long as the behavioral change is not internalized. Health education can guide this iterative process.

Promoting adherence requires a good and open relationship between physiotherapist and patient (Sluijs and Knibbe, 1991) Feedback and guidance by the therapist was found to be essential for adherence (Bø et al., 1990; Cammu et al., 1991; Hahn et al., 1993; Mouritsen et al., 1991; Sluijs and Knibbe, 1991; Wall and Davidson, 1992; Wilson et al., 1987). PFME training programs conducted by PFME therapists were found to be more effective than home exercise programs (Bø et al., 1990; Cammu et al., 1991; Wall and Davidson, 1992; Wilson et al., 1987). It is expected that an enthusiastic and devoted PFME therapist, personally giving positive feedback and reinforcement about the performance of PFMEs and paying attention to specific adherence problems, is likely to promote adherence.

Sex-specific aspects appeared to influence most determinants of adherence to PFME therapy as well. For example, our society emphasizes social roles in which women feel highly responsible for taking care of important others before taking care of themselves (Toner and Akman, 2000; Van Bavel and Wagemakers, 1996). Such caring activities often demand so much of women that there is no time and energy left for themselves, let alone for doing PFMEs (Lagro-Janssen et al., 1994). Other sex-specific aspects included associations with sexuality and the pelvic floor as an unconscious bodily area, because the social taboo on the body and female sexuality in combination with the taboo on incontinence, make the pelvic floor an area of silence and unawareness (Janetzky, 1993; Toner and Akman, 2000). In addition, women traumatized by, for example, sexual abuse often dissociate from their pelvic floor (Janetzky, 1993; LagroJanssen et al., 1994). These aspects, as well as positive or negative associations with sexuality elicited by doing PFMEs, affect adherence to PFME therapy. 
Knowledge has been identified explicitly as a determinant in relation to the precontemplation stage (Prochaska et al., 1992). In addition, implicitly, knowledge was recognized as a factor influencing the determinants beliefs, self-care strategies, attitude, outcome expectations, values, skills, and the barriers of fluctuations in the effects of PFMEs and muscle pain. Thus, knowledge plays a role in all stages and influences many determinants. Likewise, barriers play a role in different motivational stages as well.

\section{INTERVENTION MAPPING}

\section{Matrix of proximal program objectives}

This first step in IM provides the foundation for the program development, by specifying who will change as a result of the program. This process leads to the formulation of proximal program objectives, statements of what must be learned by the target group in order to impact the determinants of adherence behavior identified in the needs assessment.

\section{Specifying performance objectives}

The specific behaviors trained in PFME therapy were translated into four performance objectives for adherence to PFME therapy (Berghmans et al., 1998b; Bø et al., 1990; Bø, 1995a; Lagro-Janssen et al., 1995; Miller et al., 1998; Mouritsen et al., 1991; Nygaard et al., 1996; Payne, 2000; Versprille-Fischer, 1995; Wyman et al., 1998). The behavioral objectives that should be achieved by the intervention are:

1) Perform PFMEs regularly as advised by the therapist: perform 10 slow twitch contractions (10-30 secs.) and 10 fast twitch contractions ( $2-3$ secs.), both five times a day, each contraction being followed by relaxation. Increase repetitions in each session to the point of fatigue and incorporate different starting positions and functional activities into the exercise program. Check contractions with self-palpation.

2) Perform a correct toileting and drinking behavior: Sit straight on the toilet and relax the pelvic floor muscles during voiding, and drink 1500 to 2000 milliliters per day. Women with a deviant voiding frequency should train their bladder by timing their voidings during the day until a normal frequency of seven voidings a day is reached. 
3) Using the 'knack'; prevent wet episodes by contracting the pelvic floor muscles when feeling a sudden urge to void (urge symptoms) or when coughing, sneezing or laughing (stress symptoms).

4) Automatically and subconsciously use the pelvic floor muscles, especially during moments of abdominal pressure, in the daily posture and movement pattern to prevent leakage, thereby using a correct body posture and breathing technique.

These performance objectives are important for both adherence during and after therapy, as the latter requires the first. For example, it is unlikely that women who are not doing PFMEs on a regular daily basis during the period of therapy, will do so afterwards. In addition, after a relapse, women need to be able to restart and follow through with these behaviors.

Furthermore, it should be noted that the objective to do approximately 100 slow and fast twitch PFMEs per day must be seen as a rough guideline. It is still unclear what intensity, frequency or duration of PFMEs is most effective. Individual differences may require a different exercise regimen and when the condition of the pelvic floor muscles has recovered through exercising, a lower exercise frequency or intensity may be equally effective in maintaining the condition of the pelvic floor (Berghmans et al., 1998b; Bø, 1995b; Wells, 1990).

\section{Selecting determinants}

The needs assessment provided information on many determinants associated with adherence to PFME therapy. In this IM task, the importance and changeability of these determinants was assessed on the basis of empirical evidence and in the light of the formulated performance objectives. Table 1 shows that most of the identified determinants were mentioned in both literature sources and interviews and some in either one of the sources. But barriers and feedback were mentioned most, while there was no evidence that some determinants were more important than others. Therefore, we decided to focus on all identified determinants in the program using targeted communication to enable women to select the information parts that were most appropriate to their personal adherence problems.

The changeability of the determinants was assessed by categorizing the determinants as either personal or external ones. Personal determinants are factors under the individual's direct control, while external determinants are the social or structural factors outside the individual that directly influence the desired health behavior. When 
external determinants, such as social norms, are very difficult to change, the alternative is to deal with them indirectly through personal determinants (Bartholomew et al., 2001). Thus, the external determinant social norms is rephrased into the personal determinant perceived social norms. The determinants social support and feedback are categorized as both personal (woman) and external (physiotherapist).

\section{Considering whether to differentiate the target population}

The target population for this intervention consisted of women with symptoms of stress, urge or mixed UI. The literature does not describe any differences in performance objectives or proposed determinants between these three diagnostic groups. However, several objectives could be relevant for some women but not for others. For example, knowing how to toilet correctly and drink enough is necessary for the whole target group, but only women with deviant behavior need to change it. Still, no separate programs were developed, but the program was designed in such a way that every woman could recognize her own behavioral needs. This means that every part of the program refers explicitly to the individuals or behavioral need to which it is addressed (see paragraphs 3.2 and 3.2.1).

\section{Creating a matrix of proximal program objectives}

A matrix of proximal program objectives was created by relating the performance objectives (rows) to the determinants (columns) (Table 2). When a determinant was not applicable with respect to an objective, that cell remained empty. Answering the question 'What does the target group need to learn with regard to a specific personal determinant in order to achieve the performance objective?' revealed the learning objectives. Answering the question 'What needs to change in the environment in order for the target group to achieve the performance objective?' revealed the change objectives (Bartholomew et al., 2001). For example, the learning objective 'relaxes oneself during stressful or busy periods' results from the answer to the question 'What does the target group need to learn with regard to self-efficacy in order to perform PFMEs regularly?' The idea behind this is that when women learn to take some relaxing moments for themselves each day, there is enough opportunity left for exercising. 
Table 2. Matrix of proximal program objectives for promoting adherence to PFME therapy, examples of learning and change objectives

\begin{tabular}{|c|c|c|c|c|c|c|}
\hline \multirow{2}{*}{$\begin{array}{l}\text { Performance } \\
\text { objectives }\end{array}$} & \multicolumn{3}{|c|}{ Personal determinants } & \multicolumn{3}{|c|}{ External determinants } \\
\hline & Knowledge & $\begin{array}{l}\text { Attitude, values, beliefs, } \\
\text { outcome expectations, } \\
\text { risk perception }\end{array}$ & $\begin{array}{l}\text { Skills, self-eflicacy, } \\
\text { barriers }\end{array}$ & Modeling & $\begin{array}{l}\text { Social suppert } \\
\text { Feedback (woman) }\end{array}$ & $\begin{array}{l}\text { Social support } \\
\text { Feedback (PFME } \\
\text { therapist) }\end{array}$ \\
\hline $\begin{array}{l}\text { 1. Performs PFME } \\
\text { regularly as } \\
\text { advised by } \\
\text { physiotherapist }\end{array}$ & $\begin{array}{l}\text { Describes: } \\
\text { - risk factors for UI } \\
\text { - how PFM function } \\
\text { - what PFM are } \\
\text { - how to contract and } \\
\text { relax PFM } \\
\text { - situations that influence } \\
\text { the PFM and diminish the } \\
\text { effect of PFMEs } \\
\text { - what personal barriers } \\
\text { prevent regular PFMEs } \\
\text { - the relationship between } \\
\text { the taboo on incontinence } \\
\text { and sexuality and self- } \\
\text { esteem and body esteem, } \\
\text { and the effect on doing } \\
\text { PFMEs } \\
\text { - type and frequency of } \\
\text { PFMEs needed to improve } \\
\text { PFM function } \\
\text { - societal influence on } \\
\text { female roles } \\
\text { Explains: } \\
\text { - that doing PFMEs helps } \\
\text { regaining control over } \\
\text { bladder and body } \\
\text { - that UI is a common } \\
\text { complaint of adult women } \\
\text { and older men }\end{array}$ & $\begin{array}{l}\text { Expects: } \\
\text { - that regular PFMEs help } \\
\text { decrease UI symptoms } \\
\text { - that UI gets worse when } \\
\text { not managed } \\
\text { - expects that doing PFMEs } \\
\text { can improve feelings of } \\
\text { control and ones self- } \\
\text { esteem and body esteem } \\
\text { States: } \\
\text { - that UI can be managed } \\
\text { - that PFMEs can be } \\
\text { successful in spite of age } \\
\text { - that it is necessary to set } \\
\text { realistic goals } \\
\text { - that PFMEs can be } \\
\text { performed during other } \\
\text { activities } \\
\text { - that doing PFMEs only } \\
\text { takes a few minutes a day } \\
\text { - that it is always } \\
\text { useful to restart doing } \\
\text { PFMEs after a relapse } \\
\text { - that it takes two months } \\
\text { before results can be } \\
\text { expected }\end{array}$ & $\begin{array}{l}\text { - demonstrates correct } \\
\text { PFMEs } \\
\text { - performs 5x10 fast \& } \\
\text { 5x10 slow PFMEs per day } \\
\text { - determines a few minutes } \\
\text { a day to do PFMEs } \\
\text { - performs PFMEs during } \\
\text { other activities } \\
\text { - reminds oneself of doing } \\
\text { PFMEs regularly } \\
\text { - makes an exereise } \\
\text { schedule for nornal } \\
\text { and for stressful } \\
\text { or irregular periods } \\
\text { - relaxes oneself during } \\
\text { stressful or busy periods } \\
\text { - restarts regular PFMEs } \\
\text { after a relapse } \\
\text { - copes with uncertain or } \\
\text { uncomfortable feelings } \\
\text { about the body or self by } \\
\text { discussing these feelings } \\
\text { with the physiotherapist } \\
\text { - decides that is not } \\
\text { necessary to feel } \\
\text { responsible for everyone } \\
\text { all the time and that } \\
\text { reserving time for } \\
\text { oneself facilitates } \\
\text { performing regular PFMEs } \\
\text { - focuses on one's own } \\
\text { body at least once a day }\end{array}$ & $\begin{array}{l}\text { Copies: } \\
\text { - behavior of other } \\
\text { women who perform } \\
\text { PFMEs regularly } \\
\text { - behavior of other } \\
\text { women who continue } \\
\text { with regular PFMEs } \\
\text { unless barriers } \\
\text { Recognizes: } \\
\text { - that other women } \\
\text { benefit from regular } \\
\text { PFMEs } \\
\text { - how other } \\
\text { women are able to } \\
\text { restart exereising after } \\
\text { a relapse }\end{array}$ & $\begin{array}{l}\text { Discusses: } \\
\text { - doing PFMEs and asks } \\
\text { for support with others } \\
\text { - effects with } \\
\text { physiotherapist } \\
\text { - insecurities, worries } \\
\text { and barriers that } \\
\text { prevent exereising } \\
\text { with physiotherapist } \\
\text { - a relapse in exercising } \\
\text { with the physiotherapist } \\
\text { Recognizes: } \\
\text { - effects of doing PFMEs } \\
\text { regularly in terms of } \\
\text { personal goals that are } \\
\text { reached } \\
\text { - a correct PFM } \\
\text { contraction } \\
\text { - that reserving time } \\
\text { for oneself makes } \\
\text { doing PFMEs easier }\end{array}$ & $\begin{array}{l}\text { Therapist: } \\
\text { - evaluates effects of } \\
\text { doing PFMEs regularly } \\
\text { in terms of goals that } \\
\text { are reached } \\
\text { - discusses problems } \\
\text { with regular } \\
\text { exereising and } \\
\text { promotes women's } \\
\text { own solutions } \\
\text { - gives positive } \\
\text { feedback on correct } \\
\text { contraction } \\
\text { - reinforces good } \\
\text { adherence behavior } \\
\text { - promotes woman to } \\
\text { read self-help guide } \\
\text { - reattributes relapses to } \\
\text { mistakes to learn from } \\
\text { and stimulates to } \\
\text { restart exereising }\end{array}$ \\
\hline
\end{tabular}




\begin{tabular}{|c|c|c|c|c|c|c|}
\hline $\begin{array}{l}\text { 2. Performs a } \\
\text { correct drinking } \\
\text { and toileting } \\
\text { behavior }\end{array}$ & $\begin{array}{l}\text { Describes: } \\
\text { - how the bladder functions } \\
\text { - normal voiding frequency } \\
\text { - healthy drinking amount } \\
\text { - how to toilet correctly in } \\
\text { terms of posture and } \\
\text { relaxation }\end{array}$ & $\begin{array}{l}\text { Expects: } \\
\text { - that toileting less or more } \\
\text { often than seven times a } \\
\text { day can cause } \\
\text { incontinence problems } \\
\text { - that drinking } 1500-2000 \\
\text { milliliters per day is } \\
\text { necessary to prevent } \\
\text { kidney and bladder } \\
\text { problems } \\
\text { - that training the bladder, } \\
\text { drinking enough and } \\
\text { toileting correctly helps } \\
\text { reducing UI symptoms }\end{array}$ & $\begin{array}{l}\text { - trains a normal voiding } \\
\text { frequency of seven } \\
\text { voidings per day } \\
\text { - drinks } 1500-2000 \\
\text { milliliters per day } \\
\text { - reminds oneself to drink } \\
\text { enough and void seven } \\
\text { times a day } \\
\text { - pays attention to a correct } \\
\text { posture on the toilet } \\
\text { - takes time to void unless } \\
\text { internalized norm of } \\
\text { being needed all the time }\end{array}$ & $\begin{array}{l}\text { Copies: } \\
\text { - behavior of other } \\
\text { wornen who regain } \\
\text { bladder control by } \\
\text { training a normal } \\
\text { voiding frequency and } \\
\text { drinking enough } \\
\text { - correct toilet behavior } \\
\text { of other wornen }\end{array}$ & $\begin{array}{l}\text { Discusses: } \\
\text { - problems with } \\
\text { drinking, toileting and } \\
\text { voiding behavior with } \\
\text { physiotherapist } \\
\text { Evaluates: } \\
\text { - effects of own voiding } \\
\text { and drinking and } \\
\text { toileting behavior } \\
\text { in terms of goals that } \\
\text { are reached } \\
\text { - effects of using } \\
\text { correct posture on } \\
\text { toilet in terms of goals } \\
\text { that are reached }\end{array}$ & $\begin{array}{l}\text { Therapist: } \\
\text { - evaluates voiding and } \\
\text { drinking behavior in } \\
\text { terms of goals that are } \\
\text { reached } \\
\text { - discusses problems } \\
\text { with drinking and } \\
\text { toileting behavior } \\
\text { - gives positive feedback } \\
\text { on correct drinking. } \\
\text { toileting and voiding } \\
\text { behavior } \\
\text { - reinforces good } \\
\text { adherence behavior }\end{array}$ \\
\hline $\begin{array}{l}\text { 3. Prevents wet } \\
\text { episodes by } \\
\text { contracting PFM } \\
\text { during moments } \\
\text { of urgency or } \\
\text { abdominal } \\
\text { pressure }\end{array}$ & $\begin{array}{l}\text { Describes: } \\
\text { - effect of PFM contraction } \\
\text { on bladder relaxation } \\
\text { - situations when } \\
\text { PFM contractions are } \\
\text { needed to prevent leakage }\end{array}$ & $\begin{array}{l}\text { Expects: } \\
\text { - that contracting } \\
\text { the PFM during moments } \\
\text { of urgency or abdominal } \\
\text { pressure can prevent } \\
\text { leakage }\end{array}$ & $\begin{array}{l}\text { - contracts the PFM during } \\
\text { moments of urgency or } \\
\text { abdominal pressure } \\
\text { - delays toileting by } \\
\text { holding an urge with a } \\
\text { PFM contraction }\end{array}$ & & $\begin{array}{l}\text { Evaluates: } \\
\text { - effects of contracting } \\
\text { the PFM on leakage } \\
\text { during moments of } \\
\text { urgency or abdominal } \\
\text { pressure }\end{array}$ & $\begin{array}{l}\text { Therapist: } \\
\text { - evaluates if contraction of } \\
\text { PFM does prevent leakage } \\
\text { during moments of } \\
\text { urgency or abdominal } \\
\text { pressure } \\
\text { - gives positive } \\
\text { feedback on correct } \\
\text { prevention of leakage } \\
\text { by PFM contractions }\end{array}$ \\
\hline $\begin{array}{l}\text { 4. Automatically } \\
\text { uses the PFM } \\
\text { in daily posture } \\
\text { and movement } \\
\text { pattern; uses a } \\
\text { correct body } \\
\text { posture and } \\
\text { breathing } \\
\text { technique }\end{array}$ & $\begin{array}{l}\text { Describes: } \\
\text { - effect of body posture on } \\
\text { PFM and abdominal } \\
\text { pressure } \\
\text { - correct body posture and } \\
\text { movement needed for a } \\
\text { functional use of the PFM } \\
\text { - correct belly breathing } \\
\text { technique }\end{array}$ & $\begin{array}{l}\text { Expects: } \\
\text { - that a correct body posture } \\
\text { enables PFM contraction } \\
\text { - that through regular } \\
\text { exercising the functional } \\
\text { use of the PFM can } \\
\text { become automatized and } \\
\text { unconscious }\end{array}$ & $\begin{array}{l}\text { - uses the PFM functionally } \\
\text { in daily posture and } \\
\text { movement pattem } \\
\text { - corrects one's body } \\
\text { posture when necessary } \\
\text { - breaths correctly, thus } \\
\text { breathing out when } \\
\text { contracting the PFM }\end{array}$ & & $\begin{array}{l}\text { Evaluates: } \\
\text { - effect of the functional use } \\
\text { of the PFM on feelings of } \\
\text { control, body eateem and } \\
\text { self-esteem }\end{array}$ & $\begin{array}{l}\text { Therapist: } \\
\text { - evaluates the effect of } \\
\text { the functional use of } \\
\text { the PFM on feelings of } \\
\text { control, body esteem and } \\
\text { self-esteem } \\
\text { - gives positive foedback on } \\
\text { correct functional use of } \\
\text { PFM in the daily posture } \\
\text { and movement pattem } \\
\text { - reinforces good } \\
\text { ofherence behavior }\end{array}$ \\
\hline
\end{tabular}

$\mathrm{UI}=$ urinary incontinence

PFM $=$ pelvic floor muscles

PFMEs = pelvic floor muscle exercises 


\section{Rationale of the program}

In Step 2 of IM, theoretical methods and practical strategies were formulated by answering the question 'How can the learning and change objectives be accomplished?' (Bartholomew et al., 2001). Here, the literature provided theoretical and empirical evidence on the effectiveness of methods and strategies in tackling adherence problems. In Step 3, the program was designed. Although we worked through steps 2 and 3 seperately, these two development steps are described simultaneously for the purpose of conciseness of description of the program and its components. Before describing the content of the program, the theoretical rationale is described, which was based on the educational quality principles stated by Mullen and colleagues (1985).

The rationale of the program was threefold. Firstly, the Transtheoretical model was used, which combines various behavioral change theories. Following the phases of behavioral change, a patient needs to understand the relationship between the advised behavior and the health problem, to be able to weigh positive and negative outcomes of the advised behavior, to be able to tackle determinants hampering following the advised behavior, to implement and follow through with the advised behavior in daily life, and to prevent a relapse (Prochaska et al., 1992). Secondly, the Self-Regulation Theory (Leventhal and Cameron, 1987; Leventhal et al., 1998) was used to provide insight in adherence as self-management behavior. This theory states that active participation of patients is necessary to promote adherence. To give patients more control over their lives and more faith in their own abilities, attention must be paid to patients' cognitive representations of the illness in relation to coping with the illness and appraisal of the outcomes of behavioral change. The representation of the illness is based on current symptoms, previous experiences with illness and personal views of health and illness originating from social and cultural influences. This representation also influences the self-care strategies of patients. To appraise the outcomes of adherence behavior, patients need to evaluate their representations, self-care strategies and outcomes. This, in turn, could enhance their feelings of self-confidence and effectiveness in terms of adherence behavior. Thirdly, to stimulate recognition and acknowledgement of behavioral and informational needs, the principle of targeted communication was used as a means to promote adherence behavior using three starting points (Kreuter et al., 2000; Sluijs and Knibbe, 1991). Firstly, by designing the program in such a way that women could find appropriate answers to their own changing needs for information. Secondly, by targeting information for specific 
segments of the population, such as women who had experienced sexual violence. Thirdly, by operationalizing the principles of sex-specific health care (formerly called Women's Health Care) for health education (Wagemakers and Van Bavel, 1998). This involved paying attention to women's perception of their pelvic floor, to body esteem and self-esteem, to breaking down sex stereotypes and medicalization, and to making explicit the relationship between women's socialization, social position and gender role, and their adherence behavior (Janetzky, 1993; Toner and Akman, 2000; Van Bavel and Wagemakers, 1996; Wagemakers and Van Bavel, 1998; see Appendix A).

To be effective, the design and format of a health education program needs to fit with the target population and with the setting in which it will be used (Bartholomew et al., 2001), in this case, individual PFME therapy in private physiotherapy practices. Furthermore, the program should be applicable for use both during and after the PFME therapy without demanding extra session time. Therefore, we chose written health education in the form of a self-help guide for patients (Alewijnse, 1997) and structured oral education in the form of a counseling scheme for PFME therapists. This scheme stimulates PFME therapists to orally support the information of the guide and specifically to give feedback and reinforce adherence behavior.

\section{What components build the program?}

The description of the program of the self-help guide and counseling scheme follows the stages of the Transtheoretical model as they logically follow the iterative phases of the physiotherapeutic treatment plan (Verhulst et al., 1994). Table 3 presents the content of the self-help guide and the corresponding counseling tasks for physiotherapists along the motivational stages of change. The counseling scheme stimulates the systematic addressing of issues, which should have a positive influence on adherence. To help women move from one stage to another requires addressing those learning and change objectives that correspond to the determinants of that particular stage by applying effective methods and their operationalization in strategies.

Overall, principles of self-management, targeted communication and sex-specific health care are reflected in the self-help guide through several strategies. The self-help guide addresses women individually and provides room for personal experiences and perceptions. This means that, for example, the personal choice process of women is promoted by making use of filling-in tasks to stimulate women in deciding to be 
adherent, and that women are asked about their experiences to stimulate selfevaluation. Three role models illustrate the information and provide real-life experiences with adherence to PFME therapy, which were derived from the group discussions and interviews. The models are female representatives of 34,51 and 76 years of age with symptoms of UI who express their experiences while going through each of the motivational stages. In addition, the self-help guide lists addresses for further consultation and a list of books about incontinence and related health problems.

\section{From precontemplation via contemplation to preparation.}

PFME therapy starts with an anamnestic, a diagnostic and a teaching process. In this stage, women need to be made aware of the relation between adherence to PFME therapy and UI, and between their self-care strategies and symptoms. Thus, the learning objectives for the determinants knowledge and beliefs needed to be addressed through the methods of successful communication and modeling (Bandura, 1986) by using both oral and written information. To make sure that teaching messages are adopted and that women feel free to discuss their worries and problems, it is essential that PFME therapists create an open and safe atmosphere in the therapy (Kok and Bouter, 1990; Sluijs and Knibbe, 1991). The counseling scheme stimulates therapists to explore beliefs and illness representations about incontinence, the impact of symptoms on physical and psychosocial well-being, and women's self-care strategies. Furthermore, therapists are stimulated to discuss with women what PFME therapy involves and what is expected from them in terms of adherence behavior, and to explore possible barriers, such as emotions or personal situations that may prevent information processing (Table 3).

The self-help guide supports this teaching process by repeating the factual information about (1) incontinence, anatomy and physiology of pelvic floor muscles and bladder, (2) the continence mechanism, correct toileting and drinking behavior, (3) what women can expect from PFME therapy and other management options, and (4) how popular lay self-care strategies such as reduced fluid intake and frequent voiding may aggravate urge symptoms. All kinds of myths and general expectations about UI and PFME therapy are accompanied by facts to motivate women for PFME therapy. For example, the stereotypical myth that it is normal for aging women to become incontinent is accompanied by the fact that incontinence is merely a complaint of women of all ages but that imaging in commercials gives the impression that it solely a complaint of older women. In addition, the myth that only women are affected by incontinence is accompanied by the fact that it is a common complaint of older men 
Table 3. Content of the self-help guide along the motivational stages of change and counseling tasks for PFME therapists.

\begin{tabular}{l|l|}
\hline $\begin{array}{l}\text { Motivational } \\
\text { stages }\end{array}$ & Chapters of self-help guide \\
\hline $\begin{array}{l}\text { Precontemplation } \\
\text { \& Contemplation }\end{array}$ & $\begin{array}{l}\text { 1. Urinary incontinence: PFME therapy helps! } \\
\text { What's in this guide? Who is who in this } \\
\text { guide? }\end{array}$ \\
Contemplation & $\begin{array}{l}\text { 2. Urinary incontinence: You can help } \\
\text { yourself? }\end{array}$
\end{tabular}

Counseling tasks for PFME therapist

What is PFME therapy, what can you do and when can you expect results?

Preparation

Action

Precontemplation

\& Contemplation

Preparation

Preparation

\& Action

Preparation

Action \&

Maintenance \&

Relapse

prevention

(Pre)contemplation

(Pre)contemplation

\& Preparation

Maintenance

Maintenance \&

Relapse prevention
Decide when you want to do your exereises

Personal diary-pages for exercise advice

3. Tackle the problem of incontinence

Impact of urinary incontinence

Set your own goals, why doing PFME therapy?

4. Coping with incontinence

Discussing incontinence helps; drinking and toileting, toileting diary, toileting behavior

Urgency and pelvic floor muscles

Pads and hygiene

5. The challenge: make exercising a habit

What is regular exercising? What barriers prevent adherence to the advice of your physiotherapist?

Exercising, does that suit me?

Forgetting to exercise; Interruptions of daily

Routine; Emotional life events; Being too

busy; Needing social support; Am I doing it right?; Body esteem; Personal circumstances

Situations that influence urinary

incontinence and the pelvic floor muscles

6. Facts and myths about incontinence

7. Pelvic floor muscles: muscles to use What are pelvic floor muscles. Sexuality and the Pelvic floor. Muscles to exercise

Facts and myths about PFME therapy

8. A dry future

Adherence is worth while; How were you when you started and how are you today? Drinking and toileting behavior. Pelvic floor muscle exercises: how did they help you?

Self-esteem and body esteem

9. Epilogue

Addresses and further reading
Create open atmosphere, build confidence

Explore lay beliefs, self-care strategies, explain relation behavior and symptoms and content of PFME therapy

Weigh pros and cons of exercising, discuss personalized therapy goals and behavioral needs in terms of exercising and toileting and drinking behavior

Contract start of adherence behavior, reinforce selfmanagement behavior Explore adherence behavior Discuss personal barriers for adherence and personal solutions

Reinforce skills and selfefficacy regarding adherence

Teach internal feedback

Teach facts about incontinence, pelvic floor muscles and the exercises

Stimulate patient to work from simple to complex behavioral goals

Evaluate and reinforce progress

Promote evaluative filling-in tasks

Reinforce skills, self-efficacy and self-esteem

Reattribute relapses in positive terms

Discuss risk situations for relapse 
and adult women. The expectation that PFME therapy makes you definitely dry in two months is accompanied by the fact that PFME therapy requires active participation in terms of adherence behavior, which requires not only attending the therapy sessions but also practicing at home during and after therapy (Table 3, sections 1, 2, 4, 6 and 7).

\section{From preparation to action.}

In this stage, positive and negative consequences and outcome expectations of adherence to PFME therapy are explored. The self-help guide provides factual information about possible influences of UI symptoms on daily life and realistic outcomes of PFME therapy. Methods such as active information processing, risk perception, contracting and goal setting are operationalized in terms of four filling-in tasks in the self-help guide (Bandura, 1986; Bassett and Petrie, 1999; Janz and Becker, 1984; McGuire, 1985; Strecher et al., 1995). Women are first stimulated to think about what impact and how much impact incontinence symptoms have on their psychosocial wellbeing and on activities such as working, choice of clothing, doing sports, having sex, going out, and traveling. By writing this down, the actual impact of symptoms on daily life becomes visible and it is expected that this awareness motivates women to adhere to PFME therapy. The second task asks women to fill in positive and negative consequences and outcome expectations of adherence and non-adherence to PFME therapy for the short term and long term in a decision-making matrix. Then, women are encouraged to take their own responsibility and decide to adhere to PFME therapy by actually contracting themselves by writing down the date from which to start adhering to the advice. Setting this date also makes future evaluation possible of effects in terms of accomplished goals. Finally, women are asked to write down goals in terms of activities that they want to do again after a certain period without being troubled by incontinence symptoms or in terms of psychosocial wellbeing such as regaining self-esteem. Counseling by the therapist is directed towards discussing the above-mentioned processes to explore personal experiences, possibilities and wishes and to help women set realistic and personal goals (Table 3, sections 2, 3 and 7).

In both the self-help guide and the counseling scheme all possible barriers affecting adequate integration of adherence behavior in daily life are discussed and tackled (Table 3, section 5). Many examples illustrate possible situations in which adherence can be difficult, and practical advice is offered in the form of information and tips. For example, it is explained that many women fail to adhere to the advice because of simply forgetting or as a result of changes in their daily routine or emotional situations. The self-help guide offers reminders in the form of stickers, an exercise schedule to 
fill-in, as well as diary pages to write down the personal exercise advice of the therapist after each therapy session. These pages may function as a reminder during and after the PFME therapy (Marlatt and Gordon, 1985).

For the situation 'being too busy with work and/or caring activities,' first the relationship between women's strong feelings of responsibility for caring of others and their socialization process is made explicit by explaining that in our society social roles are emphasized in which women should feel great responsibility for taking care of important others before taking care of themselves (Toner and Akman, 2000; Van Bavel and Wagemakers, 1996). Then, the following advice was given: 'Try to save some leisure time for yourself every day and do whatever you like. This may help you relax and realize that you don't need to be there for everyone at every moment. At the same time, you can also see doing PFMEs as taking a moment for yourself which can help you relax during a busy day. Make it easy for yourself. Choose waiting moments for doing PFMEs, such as while waiting for a bus, for the printer of your computer, for a red traffic light or for the cash desk in the shop.'

For the personalized determinant feedback, the self-help guide provides tips to encourage women to evaluate the effects of the therapy on symptoms and aspects of quality of life for themselves: 'Stimulate yourself by thinking about the goals you have already reached instead of the goals you haven't reached yet, and reward yourself; 'When you are uncertain about doing the PFMEs correctly, always ask your therapist to check your performance.' Women are also taught to check for correct contractions themselves by, for example, inserting one or two fingers in their vagina to check whether they can feel a contraction.

In relation to this biological feedback monitoring, the guide pays special attention to body esteem, as PFME therapy can raise associations with sexuality that can either be experienced as negative or positive. Information is given about the influence of negative sexual experiences such as sexual violence, which may result in dissociation of the pelvic floor, hampering adherence. For those women, PFME therapy can be extremely difficult or may cause secondary traumas. Such women are asked to discuss their feelings with their therapist and/or to seek specific professional help for their sexual traumas. Finally, women are asked to think about what personal situations hamper their adherence to PFME therapy. In another filling-in task, they can write down these situations and their personal solutions. The counseling scheme stimulates PFME therapists to discuss possible barriers for adherence that women encounter and to help women find personal solutions. 


\section{From action to maintenance}

PFME therapists teach women how to contract and relax their pelvic floor muscles, how to integrate the use of the pelvic floor muscles in functional activities, how to toilet correctly, how much to drink per day and how to unburden the pelvic floor muscles by applying a correct belly breathing technique and body posture. They stimulate active learning by shaping the new behaviors in simpler units, explore skills and self-efficacy regarding performance and reinforce integration of adherence behavior by evaluating and appraising progress (Bandura, 1986; Clark and Dodge, 1999). The self-help guide supports this process by providing tips about when, where, how often and during which daily activities PFMEs can be done, and by stressing the importance of paying attention to a correct breathing technique and body posture while doing activities that raise abdominal pressure, such as lifting heavy objects. Women are also encouraged to listen to the needs of their own bodies so that they can decide for themselves on a suitable exercise frequency (Table 3, sections 4 and 5).

As relapses occur, the self-help guide and counseling scheme explicitly pay attention to factors influencing relapses by encouraging self-evaluation and active information processing, and by providing reattribution information (Brownell et al., 1986; Marlatt and Gordon, 1985). The self-help guide promotes self-evaluation of the outcomes of adherence behavior in terms of the personal goals that are reached by looking back at the goal-setting filling-in task and by asking if women are satisfied with their own adherence behavior. The effect of their adherence behavior on self-esteem and body esteem is evaluated by asking women to answer 12 True/False statements in a filling-in task. Statements are for example: 'I feel more comfortable with my body' and 'I don't feel ashamed anymore about my body and incontinence symptoms.'

To prevent adherence relapse because of lack of or disappointing progress, information is given about situations that temporarily aggravate UI symptoms, such as having a cold or being ill, tired or stressed, or about factors that affect control over the pelvic floor muscles such as menstruating or taking sleeping tablets. Furthermore, advice is given on the use of the guide, and especially the 'diary pages,' as a reminder to be adherent. Women are also made aware of the possibility of restarting adherence behavior after an adherence relapse and are advised to attribute relapses as a normal part of adopting new behaviors rather than as personal failures (Weiner, 1985). The counseling scheme stimulates PFME therapists to actively evaluate progress, to discuss risk situations for relapses and adherence behavior after therapy has ended (Table 3, sections 2, 3, 5, 8 and 9). 


\section{Producing the materials}

The content of the self-help guide was critically evaluated before production. An expert meeting was conducted with four PFME therapists, of whom three were also teachers in PFME therapy, a movement therapist specializing in the pelvic floor area and three employees of the National Women's Health Care Center. The operationalization of the principles for sex-specific health education in the self-help guide was also evaluated in a pilot study (Wagemakers and Van Bavel, 1998). Furthermore, the PFME therapists involved in the implementation were given the opportunity to comment on the content of the self-help guide. The most prominent comment was that the tone of the self-help guide was too paternalistic and wearisome. This could annoy women and thus affect adherence. For example, the sentence 'If you want to get rid of your wet episodes, you should really try to make the effort to exercise although that may be difficult,' was changed into 'Doing PFMEs can help you to get rid of your wet episodes. It takes some discipline in the beginning, but exercising becomes easier after a while'. From the text 'It is estimated that in the Netherlands around 675,000 women and 250,000 men have symptoms of UI, so you are not alone,' the part 'so you are not alone' was deleted. Furthermore, the therapists emphasized that the functional use of the pelvic floor muscles in the daily posture and movement pattern should be given more attention and that doing PFMEs should be explained as a method to restore and maintain the condition of the pelvic floor muscles. These evaluations helped fine-tuning of the self-help guide.

The self-help guide and reminder stickers were designed by a professional designer in close cooperation with the researcher. To make the product easy to use, to carry with you and to put on a bookshelf, an A5 format was chosen. The self-help guide contained 72 pages with a hard cover bound by a ring. An attractively colored design was chosen. Font types were large enough so that (older) women with eyesight problems could read them. Neutral white stickers and stickers with a colored design were attached to the back page. The title of the self-help guide was 'PFME therapy, practical tips and information for women who are involved in PFME therapy'. We chose not to use the term urinary incontinence on the cover to prevent feelings of shame for the condition being the reason for not using the self-help guide. The one-page counseling scheme for therapists contained - from left to right - the physiotherapeutic treatment phases, the corresponding motivational stages, the corresponding pages in the self-help guide plus the modeling text parts and several counseling tasks for the therapists. 


\section{PRACTICAL IMPLICATIONS}

This is the first study in which a full and transparent description is given of the development process of a health education program to promote adherence to PFME therapy. Program planning, development, and design are often discussed briefly in research papers, leaving implicit the processes involved in applying theoretical and empirical data to program development. We opened this black box by clearly describing the objectives of the program, the determinants, the theoretical rationale, and the operationalization of methods into strategies.

The program objectives constituted the basis for the development of the evaluation instruments that were used in a randomized controlled trial. The main questions were: Which determinants predict adherence to PFME therapy? Is the program effective in promoting adherence to PFME therapy? What is the influence of adherence on clinical outcomes? And, how do women and physiotherapists appreciate the program? This effect- and process evaluation is described in Chapters 4 and 5.

What does this detailed description of the health education program yield for health educators, health providers and researchers? So far, several authors have applied the IM approach for program development (Bartholomew et al., 1998; Bartholomew et al., 2000b; Cullen et al., 1998; Kok et al., 2000; Murray et al., 1998). What these systematic descriptions have in common, is their transparent description of the behaviors and determinants relevant to the health problem in question and the openness in theoretical and empirical evidence applied to construct the health education program. Within the context of evaluation, such elaborate descriptions allow for the analysis of possible errors, such as the rejection of the effectiveness of programs when the programs themselves were inadequate in terms of delivery (Green, 2000; Mullen et al., 1985).

Furthermore, an explicit program description can facilitate the reproducibility and diffusion of health education programs, especially when those programs have general applicability beyond the context in which they were developed and tested. Our health education program was evaluated in the South-East of The Netherlands among adult women with UI. However, the behavioral aspects of adherence to PFME therapy are general, as are most of the determinants of this particular adherence behavior, because these determinants were also found in general physiotherapy studies (Sluijs et al., 1993; Sluijs and Knibbe, 1991). Nevertheless, although the blueprint of this program is ready, it is necessary for every reproduction to take into account sociocultural 
differences and their possible effects on the determinants of adherence to PFME therapy. This implies that reproduction of this program always requires new analyses of determinants of adherence to PFME therapy among the target group involved, although it would probably be enough to do (group) interviews. Those will soon reveal whether new determinants emerge or not. It is also important to assess again which program design and what methods and strategies are applicable for the target group involved.

What have we learned from applying the IM approach for program development? Firstly, the IM planning process is always preceded by a needs assessment. However, the transition between needs assessment and Step 1 of IM may cause confusion because of overlap and the use of different terminology. In both needs assessment and IM, behavioral and environmental determinants are assessed. In a needs assessment as suggested by Green and Kreuter (1999), determinants are grouped in terms of predisposing, enabling and reinforcing factors. In IM, the first is referred to as personal and the latter two as external determinants (Bartholomew et al., 2001). We chose to group the determinants in personal and external from the beginning, to smooth the transition from needs assessment to the IM process.

Secondly, applying the IM approach to guide program development and describing this process are two different things. We discovered that describing IM Steps 2 (selecting methods and strategies) and 3 (program design) separately, results in a description of program components which leave the interested reader ignorant of what the program looks like and how it is supposed to work. Therefore, we chose to describe these two development steps simultaneously, to give a transparent description of the program and its components.

Finally, this description of the process of program development may seem a chronological process, but it is not. Applying the IM approach is an iterative process, which goes back and forth through all the steps that are in a constant process of evaluation themselves and dependent on new insights that emerge. This systematic process requires a great deal of time and attention, but it is worth the effort because it facilitates evaluation, implementation and replication of effective health education programs. 


\title{
CHAPTER 3
}

\section{Predictors of intention to adhere to physiotherapy among women with urinary incontinence}

\begin{abstract}
During the last decade, Pelvic Floor Muscle Exercise (PFME) therapy has proved its short-term efficacy among women with urinary incontinence. Long-term success with PFME therapy is hampered by non-adherence. So far, specific knowledge on determinants of adherence behavior has been scarce. A cross-sectional study was conducted to elucidate the relative importance of determinants of the intention to adhere to PFME therapy in women with urinary incontinence. Based on behavioral theories, literature research and interviews, a questionnaire measuring determinants of the intention to adhere to PFME therapy was developed. 129 women, aged 17 years or over, with symptoms of urinary incontinence, completed this questionnaire. Multiple regression analysis with backward elimination was carried out to identify determinants that predict intention. Significant predictors of the intention to adhere to PFME therapy were the amount of urinary loss per wet episode and women's perception of their ability to do the exercises as recommended under various circumstances. Building selfefficacy might be a good starting point for health education interventions aiming to promote adherence to PFME therapy.
\end{abstract}

Published, with some alterations as: D. Alewijnse, L. Mesters, J. Metsemakers, J. Adriaans and B. van den Borne (2001) Predictors of intention to adhere to physiotherapy among women with urinary incontinence. Health Education Research, 16 (2): 173-186. 


\section{INTRODUCTION}

Urinary incontinence (UI) is defined as the involuntary loss of urine, which is objectively demonstrable, with such a degree of severity that it is a social and/or hygienic problem (Abrams et al., 1988). UI is a prevailing health problem affecting many community-dwelling adults, of whom $75 \%$ are women. Overall, the estimated prevalence of UI among adult ( $>18$ years) women living in the community is $25-30 \%$, which is lower in younger age groups and higher in older age groups (Hannestad et al., 2000; Holst and Wilson, 1988; Jolleys et al., 1988; Rekers et al., 1992; Thomas et al., 1980). Stress, urge and mixed incontinence are the most frequently diagnosed types of UI (Jolleys, 1988; Lagro-Janssen, 1991). A safe and non-invasive treatment for women with these incontinence types is physiotherapeutic Pelvic Floor Muscle Exercise (PFME) therapy (Blowman et al., 1991; Klarskov et al., 1986; Wall and Davidson, 1992).

During the last decade, PFME therapy has proven its efficacy among female patients in short-term studies (up to six months follow-up) (Berghmans et al., 1996; Bø et al., 1990; Burgio et al., 1986; Burns et al., 1990; Castleden et al., 1984; Klarskov et al., 1986; Nygaard et al., 1996; Wells et al., 1991). However, little is known about longterm results of PFME therapy. Three studies have reported one-year follow-up results of PFME therapy (Ferguson et al., 1990; Lagro-Janssen et al., 1992; Mouritsen et al., 1991), three other studies followed their participants up to five years after therapy (Bø and Talseth, 1996; Cammu and Van Nylen, 1994; Lagro-Janssen and Van Weel, 1998) and one study followed them up to seven years (Hahn et al., 1993). The lowest success rates found were the result of practising alone at home or with a minimum of guidance whereas the highest success rates were the result of frequent and intensive training guided by a physiotherapist.

In general, the results of the studies above showed that success rates declined during the follow-up period. Lack of motivation and subsequent non-adherence to continuous exercise were important causes of this decline. How better adherence to PFME therapy can be achieved, needs further study. In the present study we wanted to elucidate the relative importance of determinants of adherence to PFME therapy among women with UI. 


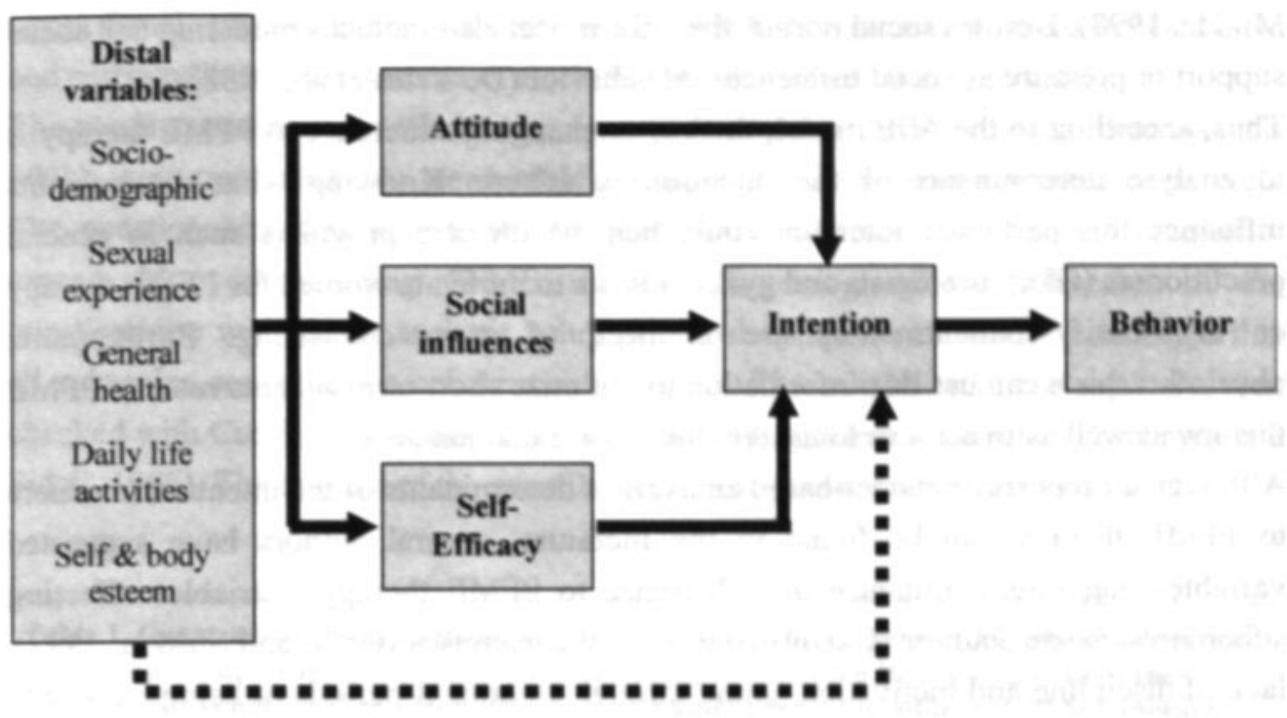

Figure 1.

The ASE model of behavioral determinants. The dotted line between distal variables and intention indicates a possible direct influence of distal variables on intention.

How to analyse determinants of adherence behavior?

To analyse determinants of adherence, the social psychological Attitude-Social influence-self-Efficacy (ASE) model was applied (De Vries et al., 1988). According to this model, behavioral change is best predicted by someone's intention to perform that behavior. The ASE model assumes that behavioral intention is determined by three types of cognitive proximal factors: attitudes, social influences and self-efficacy expectations. Distal variables such as socio-demographic, psycho-social and medical variables are expected to influence behavioral intention through the cognitive ASE variables (see Figure 1) (De Vries and Mudde, 1998; Lechner, 1998).

The ASE model has been influenced by both the Theory of Planned Behavior (TPB) (Ajzen, 1991), which also includes attitudes and social norms, and Bandura's (1986) concept of self-efficacy, constructs derived from the Social Learning Theory (SLT). Although, the TPB and the ASE model partly overlap with respect to the concepts that are integrated, they differ in the way these concepts are measured (De Vries and 
Mudde, 1998). Besides social norms, the ASE model also includes modeling and social support or pressure as social influences on behavior (De Vries et al., 1988).

Thus, according to the ASE model, the key to changing adherence to PFME therapy is to analyse determinants of the intention to adhere. Knowing what determinants influence this particular intention could help health care providers such as general practitioners (GPs), urologists and gynecologists to motivate women for PFME therapy or to identify patients who are in need of extra counseling. Furthermore, physiotherapists can use this information to optimize short-term success rates of PFME therapy as well as to achieve long-term behavioral maintenance.

Although no reports on model-based analysis of determinants of the intention to adhere to PFME therapy can be found in the literature, several authors have suggested variables that might influence the adherence to PFME therapy. Variables affecting adherence are: difficulties remembering to do the exercises (Gallo and Staskin, 1997), lack of discipline and motivation, going through emotional periods or being busy with work and childcare (Hahn et al., 1993; Lagro-Janssen et al., 1994), unfamiliarity with or feeling uncomfortable with one's pelvic floor because of the social taboos on continence and sexuality (Ashworth and Hagan, 1993; Janetzsky, 1993) or because of experiences with sexual violence (Janetzsky, 1993). In contrast, feelings of shame and experienced burden of incontinence symptoms were found to stimulate adherence to PFME therapy (Burns et al., 1993; Lagro-Janssen et al., 1994).

This paper presents cross-sectional results regarding proximal ASE and distal determinants of the intention to adhere to PFME therapy among women with UI. Data were obtained as pretest of a behavioral intervention study to promote adherence to PFME therapy.

\section{METHOD}

\section{Questionnaire}

The questionnaire was developed on the basis of a qualitative needs assessment. An extensive literature search identified important proximal ASE and distal determinants of the intention to adhere to PFME therapy. Furthermore, salient beliefs were identified through tape-recorded interviews: 15 individual and one focus-group with six women with and without PFME therapy experience, and one focus-group with 
seven female physiotherapists specialized in PFME therapy and working in Maastricht and surroundings (Van Assema et al., 1992; Morgan and Krueger, 1998).

The needs assessment revealed many aspects of proximal ASE and distal determinants which were translated into measurable concepts. Available scales were used as well. The questionnaire was pilot-tested for readability, comprehensibility and credibility among 25 women doing PFME therapy. Screening of the subscales of the questionnaire with factor analysis using principal component analysis confirmed that all subscales constituted a coherent set of items. The reliability of the subscales was checked with Cronbach's alphas, using the standardized item $\alpha$ values (Tabachnick and Fidell, 1996). The content of the questionnaire is described below and in Table 1.

Table 1. Construct, number of items, Cronbach's standardized item alpha, mean scale scores with standard deviation (SD), item range and mean and median item scores (i.s.) of the cognitive proximal ASE and distal determinants and intention to adhere to PFME therapy

\begin{tabular}{|c|c|c|c|c|}
\hline Construct, number of items, n & $\begin{array}{l}\text { Cronbach's } \\
\boldsymbol{\alpha} \text { of scale }\end{array}$ & $\begin{array}{l}\text { Mean scale } \\
\text { score (SD) }\end{array}$ & $\begin{array}{l}\text { ltem } \\
\text { range }\end{array}$ & $\begin{array}{c}\text { Mean i.s. } \\
\text { (median i.s.) }\end{array}$ \\
\hline Intention, 2 items $(n=124)$ & & $12.8(1.5)$ & $1-7$ & $6.4(6.5)$ \\
\hline Attitude & & & & \\
\hline - pros, 8 items $(n=127)$ & .83 & $41.4(5.3)$ & $1-7$ & $4.6(4.7)$ \\
\hline - cons, 5 items $(n=128)$ & .55 & $11.0(2.6)$ & $1-5$ & $2.8(2.7)$ \\
\hline \multicolumn{5}{|l|}{ Social Influence } \\
\hline - Social norms, 6 items $(n=128)$ & & $32.6(5.0)$ & $1 \cdot 7$ & $5.4(5.3)$ \\
\hline - Modeling, 1 item $(n=127)$ & & $0.3(0.5)$ & $0-10$ & $0.3(0.0)$ \\
\hline - Social support, 2 items $(n=125)$ & & $5.1(3.5)$ & $0-17 / 18$ & $2.6(2.0)$ \\
\hline \multicolumn{5}{|l|}{ Self-efficacy } \\
\hline - abilities, 9 items $(n=126)$ & .78 & $40.7(6.4)$ & $1-7$ & $4.5(4.6)$ \\
\hline - difficulties, 9 items $(n=125)$ & .87 & $35.0(9.0)$ & $1-7$ & $3.9(3.8)$ \\
\hline IQOL, 22 items $(n=128)$ & .92 & $83.9(15.8)$ & $1-5$ & $3.8(3.9)$ \\
\hline IIQ-7, 7 items $(\mathrm{n}=129)$ & .79 & $2.2(2.7)$ & $0-3$ & $0.3(0.1)$ \\
\hline Self-esteem, 12 items $(n=126)$ & .79 & $60.0(10.8)$ & $1-7$ & $5.0(5.0)$ \\
\hline Body esteem, 13 items $(n=126)$ & .84 & $62.2(12.0)$ & $1 \cdot 7$ & $4.8(4.8)$ \\
\hline Social desirability, 10 items $(n=127)$ & .63 & $80.0(12.7)$ & $0-10$ & $8.0(8.0)$ \\
\hline
\end{tabular}

\section{Intention and cognitive proximal ASE determinants}

The items 'Do you intend to follow the behavioral advice of your physiotherapist?' and 'Do you intend to exercise every day?' measured intention on seven-point scales (certainly not - yes, certainly; 1-7). The two items were summed to form one scale of intention $(\mathrm{r}=.56)$. 
Two scales were developed for attitude. The pros scale referred to what women might expect to gain by adhering to PFME therapy. These expectations were assessed on seven-point scales. For example: 'If I follow the behavioral advice, I can do my daily activities much better - much worse' (7-1). The cons scale referred to the negative consequences of adherence to PFME therapy and was assessed on five-point scales. For example: 'If I follow the behavioral advice, I will become very tired - I don't know - not tired' (5-1).

Social influence was measured with the concepts of social norms, modeling, social support and social pressure. Social norms were measured on seven-point scales by six items assessing the normative beliefs of important persons. For example: 'My friends think I should follow the therapist's advice'; 'I totally agree - I totally disagree' (7-1). The items were summed to form one sumscore. Modeling was assessed by one question asking the respondent how many persons out of 10 categories (mother, sister. Neighbor etc.) she knew who also had PFME therapy. The score ranged from 0 to 10 . Similarly, two items assessing social support asked with whom women (yes - no; 0 1) discussed their incontinence and PFME therapy $(r=.78)$. The two items were summed to form one index score (0-35). Social pressure was measured by one item asking 'Has anyone ever put pressure on you not to adhere to PFME therapy?'. Because only two respondents had ever experienced any social pressure, this item was excluded from further analysis.

Self-efficacy expectations were measured as both 'difficulties' and 'abilities' on sevenpoint scales (Bandura, 1997). In the 'abilities' scale, women's expectations of their ability to follow the behavioral advice of the physiotherapist regardless of situational demands, were measured. For example: 'I certainly can - cannot follow the behavioral advice when I am very busy' (7-1). The 'difficulties' scale referred to women's expectations of their skills regarding adherence to PFME therapy: 'Reminding myself every day to follow the behavioral advice is very easy - very difficult' (7-1).

\section{Distal variables}

An objective assessment of incontinence symptoms involved three questions regarding the frequency and amount of wet episodes and the use of protective garments, based on the PRAFAB score (Vierhout, 1990). These three aspects were noted in a seven-day diary as well (Nygaard and Holcomb, 2000; Wyman et al., 1988). Another two items measured the duration of symptoms and the type of UI. Subjective severity of incontinence symptoms was measured by the Incontinence Quality of Life scale (IQOL) (Wagner et al., 1996) and the Incontinence Impact Questionnaire (IIQ-7) 
(Uebersax et al., 1995). Higher IQOL-scores mean a better quality of life in relation to incontinence symptoms whereas higher IIQ-7-scores mean experiencing a higher impact of symptoms in daily life.

Self-esteem was measured using 12 items of one seven-point subscale of Bekker's Autonomy Questionnaire (Bekker, 1993). The subscale consisted of statements like 'I usually know quite well what I like best' (totally agree - totally disagree; 7-1). For the concept of body esteem, no existing scale suitable for women with UI was available, so a scale was developed based on the interviews and literature (Franzoi and Shields, 1984; Franzoi, 1994). A pilot-test was conducted among 15 male and 15 female university staff. Body esteem was assessed by items on seven-point scales using statements like 'I feel ashamed about my body' (totally agree - totally disagree; 1-7). Sexual abuse was assessed by asking: 'Do you have any experience of sexual harassment, rape or incest before the age of $18^{\prime}$ and a similar question focussing on the period after the age of 18 .

Subjective general health was assessed in three ways. The abbreviated 'Inventory of Subjective Health' (VOEG; Sikkel, 1980) assessed chronic physical complaints. The 13 items were summed to form one index score (0-13). Two items of the Dutch National Health Survey assessed women's perception of their health on a five-point scale from very bad to very good (1-5), and which chronic conditions they had other than UI (index, 0-26) (Van den Berg, 1992).

Social desirability was assessed using the Social Desirability scale (Visser and Breemhaar, 1989). Items such as 'If I make a mistake, I will always be prepared to admit it', were measured on a VAS scale (true -- false, 0-10), and summed to form an index score ranging from 0 to 100 . To assess socio-demographic variables, questions were asked about age, education level, insurance status, having a partner, number of children, and hours per week spent on (paid) labor, housekeeping, care for relatives or other persons, physical and other leisure activities.

\section{Participants}

The study was approved by both the Medical Ethical Committee of Maastricht University and the Maastricht University Hospital and the Review Committee of the Registration Network Family Practices (RegistratieNet Huisartspraktijken, RNH) (Metsemakers et al., 1992). The RNH consists of 23 general practices with 55 general practitioners (GPs), covering a population of around 100,000 persons, whose data on 
relevant chronic health problems and demographic variables are registered. The database was used as a sampling frame. We chose not to select on UI as most women don't seek help for this problem, and thus this is not a common registered health problem in the database. Therefore, women over 17 years old with at least one of the following risk factors for UI were selected: vaginal delivery, medical history of gynecological operations, asthma, arthritis and obesitas (Diokno et al., 1990; LagroJanssen, 1991; Milsom et al., 1993; Snooks et al., 1990; Yarnell et al., 1982). Women were recruited from the RNH in two phases. Participants were informed about the study design and were asked to confirm their agreement with the terms of the study.

In the first phase, in June 1995, nine general practices participated in the recruitment. Through their GP, 2914 women received a short recruitment questionnaire accompanied by a letter explaining that the data to be collected would be used in a study on the prevalence of UI among women with risk factors for this condition. The recruitment questionnaire consisted of items on risk factors for UI, continence status, quality of life according to continence status and use of health care resources for UI (Weijts et al., unpublished data). Women reporting UI could fill in their names and addresses if they were interested in individual PFME therapy and participation in the behavioral intervention study.

In January 1998, 198 of the initial interested women $(n=320)$ still wanted to participate. A second similar recruitment phase was then started, in which 1341 women were selected from the databases of four other general practices This phase resulted in 166 women who were willing to participate. Finally, a recruitment poster in the waiting room of the 13 participating general practices resulted in an additional 12 women. From January to October 1998, these 376 women received a letter from the researcher explaining that participation in the behavioral intervention study involved engaging in individual PFME therapy with a female physiotherapist and completing four questionnaires. The letter asked the women to make an appointment with their GP. After this letter 180 women were still prepared to participate.

The GP checked the women on the selection criteria for the behavioral intervention study. Inclusion criteria were: ability to complete questionnaires, full understanding of the Dutch language and completion of the consent form. Excluded from the study were women (total $n=47$ ) without symptoms of stress, urge or mixed UI $(n=11)$ based on their anamneses (Lagro-Janssen et al., 1995), women suffering from neurological conditions such as MS, CVA and spina bifida or suffering from venereal disease $(n=$ $5)$, women with viral infections $(n=4)$, women using medication for UI or using 
medication that enhances/influences UI $(n=1)$, women who were pregnant or within three months after delivery or women who had been operated upon for UI $(n=20)$, and women with physical impairments making PFME therapy impossible $(n=6)$. The GP also evaluated the presence of prolapse and vaginal atrophy, and the ability to voluntarily contract the pelvic floor muscles through vaginal palpation. Women with urinary tract infection were treated and included when cured.

Altogether, 47 women were excluded from the study. The remaining 133 participants received the pretest questionnaire from the GP's assistant. Four women declined to complete the questionnaire, leaving 129 women whose questionnaires were used in the present study's analyses.

\section{Statistical analyses}

Statistical analyses were performed using SPSS version 7.5. Multiple linear regression analysis with backward elimination was used to determine the significant predictors of the intention to adhere to PFME therapy. For this purpose, two-tailed tests on Pearson correlation coefficient were used to check the relation of the continuous variables with intention. Two-tailed tests on Spearman correlation coefficient were used for the ordinal variables, while independent samples t-tests were used to reveal the relation between intention and the dichotomous variables. Distal determinants that correlated significantly with intention were entered into the regression equation, together with the proximal variables. Furthermore, independent samples t-tests were used to check for differences in scores between the two recruitment phases. Recruitment phase was used as an independent variable in the regression analyses as a possible confounder, and interaction terms were checked. One by one, variables with the lowest standardized regression coefficient were eliminated from the equation using a removal p-value of .05 . We used adjusted $R^{2}$ to describe the explained variance of intention rather than $R^{2}$, as adjusted $\mathrm{R}^{2}$ corrects for both the number of independent variables used in the first regression equation $(n=12)$ and for the number of observations $(n=129)$. 


\section{RESULTS}

\section{Differences between women from the two recruitment phases}

Independent samples t-tests were used to check for differences between the participants from the two recruitment phases. Women from the first recruitment phase spent more hours taking care of others $(p<.05)$, engaging in sports activities $(p<.05)$ and following courses $(\mathrm{p}<.01)$, and they had fewer physical complaints $(\mathrm{p}<.001)$, a more positive body esteem $(p<.05)$ and a slightly more negative attitude $(p<.05)$ towards adherence to PFME therapy, than women from the second recruitment phase. No significant interaction terms were found. Women recruited in phase one or two did not differ in their intention to adhere to PFME therapy.

\section{Non-response analysis}

Of the women who responded to the recruitment and who reported having symptoms of UI but who declined to participate in the study, reasons for non-participation were collected in two ways. First, the replies in the recruitment questionnaire to the question 'If you don't want to start PFME therapy and be involved in the study, would you write down the reason why?' were summarized. Second, we telephoned the women who wanted to participate but declined after receiving the letter about the start of the study, asking if they would tell us their reasons. Altogether, 526 women mentioned a total of 659 reasons for non-participation. The most frequently mentioned reason ( 246 times; $37 \%$ ) was that their symptoms were minor or that they were not sufficiently bothered by their wet episodes to start PFME therapy. Other frequently mentioned reasons were: having other more severe health problems (94 times; 14\%), having done PFME therapy (with or without a physiotherapist) or having been operated upon for incontinence ( 82 times; $12 \%$ ), being too busy with work and/or caring for relatives (63 times; $10 \%$ ) or no longer having symptoms but being only interested in getting information about UI (30 times; $5 \%$ ). Less frequently mentioned reasons were: no interest in participation in a study, being afraid of vaginal palpation by the GP, feeling ashamed, being satisfied with the use of protective garments or medication, feeling too old to engage in PFME therapy, having no means of transport to the physiotherapist or loss of mental capacity. Fifteen women had moved to another city or had died between recruitment and the start of the study. 


\section{Distal determinants}

Socio-demographic characteristics and other distal determinants of the 129 participants are presented in Table 2 and partly in Table 1. The youngest woman was 27, the oldest 82. Most women had a partner and children, and one in three women still had children living at home. Of the $\mathbf{4 5}$ women with a paid job, $\mathbf{4 0 \%}$ worked more than three days per week. Taking care of spouse, children and/or important others, combined with household activities was taking women on average 27 hours per week. Forty-five percent of the women was involved in sports activities and $65 \%$ was taking courses, or engaged in leisure activities such as voluntary or club work. More women had a low education level as compared with the general Dutch population (www.cbs.nl, 2001). Most women were insured under the Social Health Insurance scheme.

Mean body mass index was 26.9 (SD 4.8), which is slightly above normal. Twice as many physical complaints (VOEG) were reported as found among middle-aged healthy women (M 3.5 SD 3.0; Joosten and Drop, 1987). Approximately one in three women evaluated their own health status as good, one in four as not so good, two in five as moderate, and four in five women mentioned having other chronic diseases. Experiences of sexual violence in the youth and in adult life were reported by 22 $(17.1 \%)$ and $16(12.4 \%)$ women, respectively. The majority of the women reported having symptoms of stress and mixed UI of moderate severity for several years. Most women lost droplets and dashes on several days per week, and mean weekly frequency of wet episodes was 24.5 (SD 25.0).

For $76.7 \%$ of the women, wet episodes had some negative impact on daily life activities (IIQ-7, see Table 1). The impact of the involuntary wet episodes was most keenly felt on physical activities like swimming, biking, walking and sports activities, with $42.6 \%$ experiencing some impact and $17 \%$ experiencing considerable impact on these activities. Nearly all the women $(97.7 \%)$ worried now and then about their incontinence (IQOL). Most important worries were about deterioration of symptoms when growing older $(58.9 \%)$, possible wet episodes occurring when coughing or laughing $(55 \%)$ or when feeling a sudden urgency $(40 \%)$. Women who had a lower quality of life in relation to incontinence symptoms, had more frequent wet episodes ( $r$ $=-.45, \mathrm{p}<.01)$ and assessed their health as more negative $(\mathrm{r}=.26, \mathrm{p}<.01)$ than women with higher IQOL-scores. For the IIQ-7 these correlations were nearly the same: $.40(\mathrm{p}<.01)$ and $-.29(\mathrm{p}<.01)$ respectively. IQOL and IIQ-7 scores did not correlate with the amount of urine lost per wet episode nor with the duration of symptoms. 
Table 2. Characteristies of participants, sociodemographic and other distal variables, $N=129$

Variables

Number of

Percentage of

Mean (SD)

women

population

Age

Having a partner

Having children

Number of children

Having a paid job

Hours of paid labor per week

Hours of care' per week

Hours of activities ${ }^{2}$ per week

Hours of sport per week

Education Level $(n=127)$

\section{Low \\ Medium 4}

Highs $^{\text {s }}$

Had sex education at school

Social Health Insurance

Recruitment phase 1995

Recruitment phase 1998

Body mass index

VOEG

General health status

Good
Moderate

Other chronic conditions (0-26)

Sexual violence in youth

Sexual violence in adult life

Self-diagnosis, type of UI

Stress

Urge

Mixed

Duration of symptoms $(n=127)$

less than 2 years

2.7 years

over 7 years

Amount of urinary loss

Droplets

Droplets and dashes

Dashes

Dashes and whole bladders

Whole bladders

Frequency of wet episodes

Max. 1x per week

2-7x per week

Several times a day
107

118

45

84

58

96

16

15

43

98

69

60
$55.6(10.9)$

$82.9 \%$

$91.5 \%$

$34.9 \%$

$22.0(11.3)$

$27.2(15.4)$

$65.1 \%$

$45.0 \%$

$5.6(4.8)$

$3.1(2.4)$

$75.6 \%$

$12.4 \%$

$11.8 \%$

$33.3 \%$

$76.0 \%$

$53.5 \%$

$46.5 \%$

$26.9(4.8)$

$7.2(3.2)$

$35.7 \%$

$40.3 \%$

$24.0 \%$

$17.1 \%$

$12.4 \%$

$38.8 \%$

$12.4 \%$

$48.8 \%$

$7.2(6.7)$

$8.7 \%$

$56.7 \%$

$34.6 \%$

$16.3 \%$

$58.9 \%$

$17.8 \%$

$6.2 \%$

$0.8 \%$

$20.9 \%$

$41.9 \%$

$37.2 \%$

Weekly frequency of wet episodes,

7-day diary $(\mathrm{n}=125)$

$24.5(25.0)$

1. Care activities involved hours per week spent on household and caring for (ill) important persons; 2 . Activities involved hours per week spent on taking courses, or doing club or voluntary work; 3. Low education level: completed primary school and vocational school; 4. Medium education level: completed secondary (vocational) school; 5 . High education level:

completed the highest level of secondary (vocational) school or university. 


\section{Proximal ASE determinants and intention}

Table 1 presents mean scale scores, item range, mean and median item scores and standard deviations of the proximal ASE determinants and intention to adhere to PFME therapy. Women had a slightly positive attitude (pros) towards the intention to adhere to PFME therapy. At the same time they expected some negative outcomes (cons). The mean item scores indicate that many women did not know what to think. Many women $(76.0 \%)$ were convinced that significant others would want them to follow the behavioral advice of the physiotherapist. Three in four women knew no other women engaged in PFME therapy, while the other women knew one or two women who used this therapy. Almost all women had discussed their incontinence problem $(92.2 \%)$ and the PFME therapy $(91.5 \%)$ with one or more other people, mostly their partners, daughters, female friends, sisters and mothers.

Most women expected that following the behavioral advice of the physiotherapist would be neither difficult nor easy. Not going to the toilet each time they felt an urge was expected to be slightly to very difficult by $62.2 \%$ of the women, and $56.6 \%$ of the women expected to have difficulties relaxing. The participants had slightly positive expectations about following the behavioral advice every day, as recommended, in various situations such as while working, while taking care of others or when nervous. For example, most women expected to be able to do pelvic floor muscle exercises as advised during the weekend $(80.3 \%)$ or during holidays $(64.3 \%)$. The mean item score on intention indicated that most participants had a very positive intention to adhere to PFME therapy.

\section{Relative importance of determinants of intention}

Of the distal variables, four were significant correlates of intention. Two variables dealt with UI itself: the amount of urinary loss per wet episode $(r=.218, p<.05)$ and stress UI $(\mathrm{t}=-2.363, \mathrm{p}<.05)$. Furthermore, subjective general health $(\mathrm{r}=-.211, \mathrm{p}<$ $.05)$ and hours of paid labor per week $(r=-.190, p<.05)$ correlated significantly with intention. Of the ASE variables, self-efficacy in terms of both 'abilities' $(r=.401, p<$ $.01)$ and 'difficulties' $(\mathrm{r}=.306, \mathrm{p}<.01)$, were significantly correlated with intention.

Linear multiple regression analysis with backward elimination was conducted to determine the solely important determinants of intention to adhere to PFME therapy (Table 3). The four distal determinants that significantly correlated with intention, all 
the proximal ASE determinants and recruitment group were entered as independent variables. In nine steps, the following variables with the lowest standardized regression coefficient were removed from the regression equation: 1) attitude: pros, 2) hours of paid labor per week, 3) recruitment group, 4) social support, 5) stress incontinence. 6) social norms, 7) attitude: cons, 8) subjective general health and 9) modeling. Three determinants remained which significantly predicted intention, explaining $22 \%$ of the variance in intention. A positive intention to adhere to PFME therapy could be explained by large amounts of urine lost per wet episode and a strong perceived ability to follow all the different behavioral advice (difficulties), also in various situations (abilities).

Table 3. Multiple regression analysis with backward elimination with intention as dependent variable and all independent variables entered in first equation; standardized beta-coefficients $(\beta)$, significance level $(p)$ and explained variance. $95 \%$ confidence intervals for final equation.

\begin{tabular}{llll}
\hline $\begin{array}{l}\text { Variables entered in first } \\
\text { equation }\end{array}$ & $\begin{array}{l}\beta \text { 's in first } \\
\text { equation }\end{array}$ & $\begin{array}{l}\beta \text { 's in final } \\
\text { equation }\end{array}$ & $\begin{array}{l}95 \% \text { confidence } \\
\text { interval }\end{array}$ \\
& $\mathbf{R}^{2}=.33$ & $\mathbf{R}^{2}=.24$ & \\
& $\mathbf{R}^{2}$ adjusted $=.25$ & $\mathbf{R}^{2}$ adjusted $=.22$ & \\
\hline
\end{tabular}

Distal variables

Recruitment group $-.056$

Amount of urinary loss per wet episode $.207 *$

Stress incontinence .102

Hours of paid labor per week $\quad-.057$

Subjective general health

$-.204^{*}$

Proximal ASE-variables

Attitude: pros

Attitude: cons

Social Influence

Social norms'

Modeling

Social support

Self-efficacy

Abilities

$-.080$

Difficulties

${ }^{*} \mathrm{p}<.05 ;{ }^{* *} \mathrm{p}<.01$ 


\section{DISCUSSION}

The purpose of the present study was to examine the relative importance of determinants of the intention to adhere to PFME therapy among women with symptoms of stress, urge and mixed UI. As intention is not behavior itself, results have to be interpreted with caution. However, according to the theory of the ASE model, intention has proven to be the best predictor of actual behavior (Godin and Kok, 1996), which could justify comparison with studies on determinants of adherence and tentative recommendations for practice.

Of the proximal ASE determinants, self-efficacy was the only significant predictor of intention. Positive intentions depended on the women's own perception that they would not only be able to follow the behavioral advice of the physiotherapist on a regular daily basis but also to do this under various circumstances, such as when busy, during the weekend or when not feeling well. In studies on determinants of intention of health behavior in general as well as studies on exercise behavior in particular, self-efficacy was found to be an important predictor of intention as well (Godin and Kok, 1996; Kok et al., 1991; Lechner and De Vries, 1995; Marcus and Owen, 1992; McAuley, 1993). Furthermore, self-efficacy has been found to predict adherence to physiotherapeutical exercise therapy (Sluijs and Knibbe, 1991).

A possible explanation for attitude being of no importance to intention might be that the women did not know exactly what PFME therapy involved, as was indicated by the neutral mean item scores. If one does not yet know the exact nature of a recommended behavior, it is probably very hard to evaluate the possible positive and negative outcomes of performing this particular behavior. Another explanation might be that the construct validity of the attitude questions, and especially those of the cons questions, was not optimal. It is suggested that better attitude scales should be developed.

In our study, $92.2 \%$ of the women talked about their incontinence and $91.5 \%$ about PFME therapy almost exclusively with their partners, daughters, female friends, mothers and sisters. These contacts, however, did not influence their intention. This might be explained by the fact that the women were not yet engaged in PFME therapy when filling in the questionnaire. Thus it is not likely that they were receiving social support for this therapy. Another possibility is that just talking about these topics with other women had the effect of mutual support rather than resulting in social support to promote intention. As only $25.6 \%$ of the women knew other women doing PFME therapy, $74.4 \%$ had no opportunity to benefit from modeling with respect to PFME therapy, which might explain the modest influence of modeling on intention. However, 
it could be argued that modeling did have some modest influence on intention, as it was only eliminated from the regression equation in the last step. Godin and Kok (1996) argue that there is a consistent, though small, contribution of social norms to intention and that modeling might be a better predictor of intention.

Although the theory of the ASE model assumes that the influence of distal variables on intention is mediated through the proximal variables, we found one distal variable that directly influenced intention. Women who lost large amounts of urine per wet episode had a positive intention to adhere to PFME therapy. The direct influence of distal variables on intention has also been found in a determinant study on the health risk behavior of sunbathing (Lechner and De Vries, 1997a). In other studies, women with frequent wet episodes were more likely to adhere to PFME therapy than those with fewer episodes (Burns et al., 1993) and women who were more bothered by their wet episodes had a better adherence than less bothered women (Lagro-Janssen et al., 1994). A similar relation between the perceived severity of symptoms and adherence to exercise therapy was found in physiotherapy research (Sluijs et al., 1993). Our finding cannot be explained by the mediating effect of the amount of urine lost per wet episode for the frequency of wet episodes, IQOL or IIQ-7 scores, as the amount of urine lost per wet episode was not significantly correlated with these variables. A possible explanation might be that, knowing that the larger the amounts of urine lost per wet episode the more positive the intention, having wet episodes of a dash or even a whole bladder is such a nasty experience itself that it directly influences intention rather than through the proximal ASE or other variables.

A limitation of the present study was that much of the variance in intention could not be accounted for; as only $22 \%$ of the variance in intention could be explained. The nine variables successively removed from the regression equation together only contributed 3\% to the variance in intention. Other studies have reported somewhat better predictions of intention. In a study by Lechner and De Vries (1995), $33 \%$ of the intention to start exercise behavior was explained by proximal ASE variables. And a review by Godin and Kok (1996) of the relative importance of proximal ASE variables for the intention to adopt various health behaviors, found that the proximal variables could predict an average of $42 \%$ of intention.

One of the reasons why much of the variance in intention was left unexplained in our study might be that the intention data were negatively skewed, which was caused by the fact that most women had a very positive intention to adhere to PFME therapy. This, in turn, can be explained by the fact that the women involved in the present study 
were already willing to participate in a subsequent behavioral intervention study in which they would start PFME therapy. It was to be expected that these women would also have a positive intention to adhere to this therapy. Furthermore, transformation to normality revealed the same results. Another explanation might be that the women in the present study had a strong tendency to give socially desirable answers. This may also have affected other answers on determinant questions. However, all answers would then be flawed in the positive direction, which would probably not influence the correlations between the determinants. Our overall conclusions would thus probably not have been different if our respondents had given less socially desirable answers. Furthermore, the Cronbach's alpha value of the social desirability scale was .63, which raises questions about its reliability. This is understandable, as the women in the pretest found these items very annoying and strange. Nevertheless, since adherence behavior is a socially desirable behavior, we have to be cautious in drawing conclusions.

Many of the women we approached declined to participate in our study. Most of the women who declined to be involved in the subsequent behavioral intervention study, said that they did not feel the need to start PFME therapy because they had mild symptoms of UI or were not bothered by their wet episodes. This seems to agree with our finding that women who lost large amounts of urine per episode also had a positive intention to adhere to PFME therapy. Another frequently mentioned reason was that the women were too busy with their job and/or taking care of important others. This fits in with the negative correlation we found between the number of hours of paid labor and the intention to adhere.

More insight into women's intentions to start and adhere to PFME therapy could be obtained from further studies which take into account motivational stages of behavioral change, as recent findings indicate the usefulness of incorporating motivational stages into studies of exercise behavior (Laforge et al., 1999). Care should be taken in the timing of recruitment for such studies, as the unfortunate delay between our two recruitment phases may have caused the relatively high level of non-response.

\section{Implications for practice}

The results of this study suggest that GPs, but also gynecologists and urologists, can identify women with urinary incontinence whose intention to adhere to PFME therapy is likely to be positive, by asking two questions. First, they can ask the women's 
amount of urinary loss per wet episode. Secondly, they can ask women if they feel able to perform pelvic floor muscle exercises and integrate them in their daily life activities. If a woman answers that she is losing large amounts of urine per wet episodes, and/or has a positive estimate of her self-efficacy, such a woman is likely to have a positive intention to adhere to PFME therapy. In this case, referral to a physiotherapist specialized in the field of PFME therapy is highly recommended, as success can be expected.

However, we would certainly not suggest that women with small amounts of urinary loss per wet episode should not be referred to PFME therapy. The problem is that these women are less likely to seek help. In our study, most of the women recruited had never sought help for their incontinence, even though they had moderately severe symptoms for up to 35 years. It is known that $50-75 \%$ of women with UI fail to seek help because of feelings of shame, lack of knowledge about management options or because they think that UI is an inevitable fact of life after childbirth or with increasing age (Lagro-Janssen, 1991; O'Dowd, 1993). It is thus important that health professionals ask about symptoms of UI if a woman is at increased risk. Another method of inducing women to disclose their symptoms is to display leaflets about pelvic floor muscle exercises in the waiting room. As the women hardly responded to our recruitment poster in the waiting rooms and in view of the taboo aspect of the condition, it might be better to put up posters on the doors of public toilets, explaining that PFME therapy is an effective and safe therapy. The use of models on such a posters can be recommended. For those women who are bothered by their wet episodes but are not aware of management options, this might be the right incentive to seek help for this treatable condition.

GPs might try to promote self-efficacy regarding the intention to adhere to PFME therapy. For example, many women may be expected to claim that they are too busy with daily life activities, and thus do not deem themselves capable of integrating exercises in their busy lives. In such cases, GPs can say that pelvic floor muscle exercises take very little time per day and can be performed during any kind of activity without this being noticed by others. Furthermore, good results have been reported in a study in which GPs themselves treated women with PFME therapy (Lagro-Janssen, 1991; Seim et al., 1996). Women who have no time for regular visits to a physiotherapist, could be instructed in pelvic floor muscle exercises and bladder training by their GP, during which consultations, for example, vaginal palpation with giving biofeedback might be used as method for promoting self-efficacy (Berghmans 
et al., 1998b; Lagro-Janssen et al., 1995). However, standard training of general practitioners does not include PFME therapy and several consults are necessary to evaluate progress and stimulate self-efficacy for adherence (Cammu and Van Nylen, 1994; Hahn et al., 1993; Wall and Davidson, 1992).

Physiotherapists could address aspects of self-efficacy in an open discussion in the first few sessions of PFME therapy. This means discussing issues in such a way that a woman feels comfortable discussing adherence problems with the physiotherapist. Future health education research should explore systematically which determinants stimulate women's intention to adhere to PFME therapy. Building self-efficacy might be a good starting point for health education interventions aiming to promote adherence to PFME therapy, which can be used by physiotherapists and GPs. In the subsequent intervention study, health education interventions to promote adherence to PFME therapy will be evaluated in a randomized controlled trial with the 129 women who participated in the present cross-sectional study. 


\title{
CHAPTER 4
}

\section{Predictors of long-term adherence to pelvic floor muscle exercise therapy among women with urinary incontinence}

\begin{abstract}
This study assessed predictors of long-term adherence to pelvic floor muscle exercise (PFME) therapy among women with urinary incontinence, and evaluated the effectiveness of a specific health education program supplemented to PFME therapy on adherence behavior and predictors of adherence in a longitudinal randomized controlled trial. Sequential multiple regression analyses revealed several significant predictors, that predicted $50 \%$ of variance in long-term adherence behavior. Positive intention to adhere, high short-term adherence levels and positive self-efficacy expectations significantly predicted high long-term adherence levels. Further, women with frequent weekly wet episodes before and one year after therapy were more likely to have high adherence levels one year after therapy, than women with fewer weekly losses. Thus, women seemed to adapt long-term adherence behavior to their symptoms.
\end{abstract}

Adherence to PFME therapy was very high and was not different between conditions. The health education program had no additional impact to individual contact during PFME therapy, on adherence or on predictors of adherence. However, the protocol checklist for the PFME therapy developed as a standard for all conditions, had structured therapy content, which may have optimized adherence behavior in this study. Implementation of this protocol checklist in clinical guidelines is suggested.

Mamuscript submitted in a shorter version as: Dianne Alewijnse, Ilse Mesters, Job Metsemakers, Bart van den Borne. Predictors of long-term adherence to pelvic floor muscle exercise therapy among women with urinary incontinence. 


\section{INTRODUCTION}

Urinary incontinence (UI) is a major health problem affecting many communitydwelling adults, of whom $75 \%$ are women. Overall, the estimated prevalence of UI among adult ( $>18$ years) women living in the community is $25-30 \%$, which is lower in younger age groups and higher in older age groups (Hannestad et al., 2000; Holst and Wilson, 1988; Jolleys et al., 1988; Rekers et al., 1992; Thomas et al., 1980). The high prevalence rates bring forward an enormous financial impact on the health care costs of countries. For example, in the Netherlands up to 80 million euros are spent yearly on incontinence pads alone, those used in residential homes not taken into account (Health Council, 2001; Janssen and Miltenburg, 1988). UI is associated with reduced physical, social and mental wellbeing caused by embarrassment, fear of offensive odor, and loss of self-esteem (Dugan et al., 2000; Van der Vaart et al., 2000; Wyman et al., 1990).

The International Continence Society (ICS) has defined UI as the involuntary loss of urine which is objectively demonstrable and a social or hygienic problem (Abrams et al., 1988). The three most prevalent types of UI are stress, urge and mixed UI. Stress incontinence is the complaint of involuntary loss of urine during, for instance, coughing, sneezing and physical exertion, such as sport activities and suddev changes of position. Urge incontinence is the complaint of involuntary loss of urine associated with a sudden, strong desire to void. In mixed UI, symptoms of both stress and urge incontinence are present (Abrams et al., 1988; Blaivas et al., 1997).

Today, PFME therapy has been proven effective for women with stress and mixed incontinence (Berghmans et al., 1998a; Bø, 1995a; Hay-Smith et al., 2001). Some studies reported favorable results for urge incontinence as well (Berghmans et al., 2000; Nygaard et al., 1996; Wyman et al., 1998). Therefore, PFME therapy is recommended as first-choice treatment for women with symptoms of stress, urge and mixed UI when other physical impairments causing UI have been excluded and possible urinary tract infections have been treated successfully. Overall, in studies up to $70 \%$ of the population was cured or improved after therapy (Berghmans et al., 1998a; Bø, 1995a; Burns et al., 1993; Lagro-Janssen et al., 1992; Nygaard et al., 1996), but success rates declined during follow-up as adherence to PFME therapy recommendations deteriorated. This finding can be illustrated by three studies that found that adherence, both during the period of therapy (including practicing at home) and thereafter, was a significant predictor of long-term success (Bø and Talseth, 1996; Chen et al., 1999; Lagro-Janssen and Van Weel, 1998). In one study, $62 \%$ of the 
women reported good adherence three months after therapy. This declined to $51 \%$ one year after therapy and to $39 \%$ at five-year follow-up, while in the meantime incontinence symptoms had returned or increased significantly (Lagro-Janssen et al., 1992; Lagro-Janssen and Van Weel, 1998). Chen et al. (1999) showed that during a two-year follow-up, continence success rates declined more in the groups with poor and moderate adherence than in the group that adhered well. Be and Talseth (1996) found that those who exercised three times per week or more had significantly fewer symptoms than those who exercised less frequently, five years after organized PFME training. These results raise the question why adherence levels to PFME therapy for women with UI, drop so deep.

Research on the topic of adherence behavior in physiotherapy has suggested that specific psychosocial and cognitive factors (determinants) may contribute strongly to patient adherence to exercise therapy. Furthermore, patient education programs supplementary to or incorporated in physiotherapeutic treatment addressing determinants of adherence behavior are expected to promote adherence and improve long-term therapy outcomes (Sluijs and Knibbe, 1991; Sluijs et al., 1993). Presently, there is a broad consensus among PFME therapists and researchers that long-term

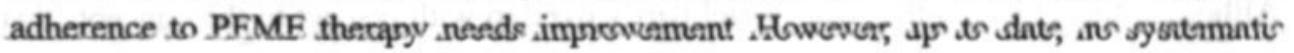
analyses have been conducted on determinants of adherence behavior to PFME therapy, nor have adherence-promoting health education interventions addressing such determinants been developed and evaluated in practice (Gallo and Staskin, 1997; Lagro-Janssen and Van Weel, 1998; Nygaard et al., 1996; Wells, 1990).

The purpose of this study was to assess the effectiveness of a specific theory-driven health education program in promoting long-term adherence to PFME therapy for women with UI, and to reveal predictors of long-term adherence to PFME therapy after therapy. The following two research questions are addressed in this paper:

1. Which are relevant predictors of long-term adherence to PFME therapy?

2. What is the influence of a specific health education program supplemented to PFME therapy on long-term adherence and predictors of adherence? 


\section{METHODS}

\section{Determinants of adherence and the health education program}

To categorize and conceptualize variables that are important in determining adherence behavior, a social cognition model was used, called the Attitude-Social influence-selfEfficacy (ASE) model (De Vries et al., 1988). According to the ASE model, behavioral change is best predicted by an individual's intention to perform that behavior. The ASE model assumes that behavioral intention is determined by three types of proximal cognitive factors: attitudes, social influences and self-efficacy expectations. Distal factors such as sociodemographic, psychological, sociocultural and medical variables, are expected to influence behavioral intention through the proximal factors. Barriers and skills play a role when behavior is actually performed, and this performance leads to a feedback process that, in turn, influences the three proximal factors (De Vries and Mudde, 1998; Lechner, 1998).

The ASE model has been influenced by both the Theory of Planned Behavior (TPB) (Ajzen, 1991), which also includes attitudes and social norms, and Bandura's (1986) concept of self-efficacy, constructs derived from the Social Learning Theory (SLT). Although, the TPB and the ASE model partly overlap with respect to the concepts that are integrated, they differ in the way these concepts are measured (De Vries and Mudde, 1998). Besides social norms, the ASE model also includes modeling and social support or pressure as social influences on behavior (De Vries et al., 1988).

The proximal and distal determinants of adherence to PFME therapy were revealed from literature and qualitative interviews (see Chapter 2). Proximal determinants were positive and negative outcome expectations regarding adherence to PFME therapy, perceived social norms, modeling, social support, and self-efficacy expectations in terms of being able to adhere to PFME therapy even under various situations. Distal determinants were lay beliefs about incontinence, self-care strategies, illness representation, cultural norms and values, risk perception, prognosis, and perceived severity of symptoms. Barriers were lack of discipline, time and energy, forgetting, stressful situations, (negative) associations with sex, perceiving the pelvic floor as an unconscious bodily area, difficulties with integrating exercises in daily life, fluctuations in effectiveness, and muscle pain (bladder). Two types of feedback play a role as well. In terms of internal feedback, progress, symptom distress or impact, selfesteem and body esteem are important for adherence behavior, while oral feedback and reinforcement from others are external feedback determinants. Three aspects influence 
many of above determinants: knowledge, sex-specific aspects, and motivation (Ashworth and Hagan, 1993; Bø et al., 1990; Bø, 1995b; Burns et al., 1993; Cammu et al., 1991; Cammu and van Nylen, 1994; Castleden et al., 1984; Dougherty et al., 1993; Gallo and Staskin, 1997; Hahn et al., 1993; Janetzky, 1993; Knibbe and Wams, 1994; Kok and Bouter, 1990; Lagro-Janssen et al., 1994 and 1995; Lagro-Janssen and Van Weel, 1998; Mantle and Versi, 1991; Mouritsen et al., 1991; Nygaard et al., 1990 and 1996; O'Dowd, 1993; Sluijs and Knibbe, 1991; Sluijs et al., 1993; Wall and Davidson, 1992; Wilson et al., 1987; Wyman et al., 1998).

As there was no evidence that some determinants of adherence were more important than others, we decided to address all identified determinants in the health education program. The development of the theory-driven health education program was based on a problem-solving approach called Intervention Mapping (Bartholomew et al., 2001), in which for each determinant specific aims were formulated and appropriate methods and strategies were selected. For example, for the method of goal setting, the strategy consisted of a filling-in task to write down personal treatment goals, and for the method of modeling, role model stories were the strategy (Bassett and Petrie, 1999; Strecher et al., 1995; see Chapter 2). Furthermore, quality principles for patient education were applied (Mullen et al., 1985). This implied among others, that information was targeted to women's needs, self-evaluation about changes in symptoms was stimulated, and reinforcement was given for positive behavior changes. In addition, we developed a minimal, a medium, and a maximum intervention that each followed the physiotherapeutic treatment plan in content, to enable investigating what intensity of health education would be necessary to promote adherence effectively. Three health education interventions were developed of which the most important components were reminders, guidance of adherence as self-management process, and structured feedback (Cramer, 1995; Leventhal and Cameron, 1987; Leventhal et al., 1998; Prochaska et al., 1992 and 1994):

1) Reminder intervention (Ri): since forgetting was most often mentioned as a barrier for adherence, this intervention consisted of a folder with information about PFME therapy and adherence behavior and several tips to remember adherence behavior. Stickers were included as reminders.

2) Reminder and Self-Help Guide intervention (R-SHGi): a guide (Alewijnse, 1997) addressing facts and myths about UI and pelvic floor muscles, coping with UI, tips to tackle all barriers hampering adherence behavior, and relapse prevention strategies to 
support the self-management process. The self-help guide also contained the stickers of the $\mathrm{Ri}$ and reminder tips.

3) Reminder, Self-Help Guide and Counseling intervention (R-SHG-Ci): identical to the R-SHGi, with the addition of a counseling scheme for physiotherapists, guiding structural oral feedback and reinforcement to promote adherence behavior.

\section{Pelvic floor muscle exercise therapy}

The content of PFME therapy and the operationalization of adherence advice as applied in this study, were based on usual care in the Netherlands and recommendations of several authors (Berghmans et al., 1998b; Berghmans et al., 2000; Bø et al., 1990; Bø, 1995a; Lagro-Janssen et al., 1995; Miller et al., 1998; Mouritsen et al., 1991; Nygaard et al., 1996; Payne, 2000; Versprille-Fischer, 1995; Wyman et al., 1998). For the purpose of this study, a protocol for the individual PFME therapy was prepared in cooperation with the participating physiotherapists as a standard for all study conditions. This written protocol checklist covered all treatment aspects that are usually applied in PFME therapy during diagnostic assessment activities, formulation of the treatment plan, commonly given patient education, treatment of exercise therapy and evaluation (Knibbe and Wams, 1994; Verhulst et al., 1994; Versprille-Fischer, 1995). A schedule for frequency of treatment sessions over time, covering nine sessions of 30 minutes each in 14 weeks, or for additional sessions up to 22 weeks, was added to the protocol. We agreed that this protocol represented usual care in a written form.

PFME therapy for stress incontinence focused on the integration of the automatic and subconscious use of the pelvic floor muscles during the daily posture and movement pattern, and on the integration of pelvic floor muscle exercises (PFMEs) in daily life. For urge and mixed incontinence, the therapy focused first on bladder training in which a normal voiding frequency of approximately seven voidings per day is trained. Second, the therapy included the same program as for stress incontinence with the addition of learning to prevent leakage when feeling an urge, by contracting the pelvic floor muscles, which inhibits the contraction of the bladder. The therapy included teaching about the anatomy and function of the bladder, the pelvic floor muscles, the continence mechanism, toilet behavior and education on the importance of adherence to the advice.

Adherence to PFME therapy was operationalized as following four behavioral advice: 
1) Perform PFMEs regularly as advised by the therapist: perform 10 slow twitch contractions (10-30 secs.) and 10 fast twitch contractions ( $2-3$ secs.), both five times a day, each contraction being followed by relaxation. Increase repetitions in each session to the point of fatigue and incorporate different starting positions and functional activities into the exercise program. Check contractions with self-palpation.

2) Perform a correct toileting and drinking behavior: Sit straight on the toilet and relax the pelvic floor muscles during voiding, and drink 1500 to 2000 milliliters per day. Women with a deviant voiding frequency should train their bladder by timing their voidings during the day until a normal frequency of seven voidings a day is reached.

3) Using the 'knack'; prevent wet episodes by contracting the pelvic floor muscles when feeling a sudden urge to void (urge symptoms) or when coughing, sneezing or laughing (stress symptoms).

4) Automatically and subconsciously use the pelvic floor muscles, especially during moments of abdominal pressure, in the daily posture and movement pattern to prevent leakage, thereby using a correct body posture and breathing technique.

These behaviors are expected to be performed during and after therapy, and after a relapse, women need to be able to restart and follow through with these behaviors. Optimal adherence during treatment is expected to be a prerequisite for long-term adherence. For example, it is unlikely that women who are not doing PFMEs on a regular daily basis during the period of therapy, will do so afterwards.

Furthermore, it should be noted that the objective of doing approximately 100 slow and fast twitch PFMEs per day, must be seen as a rough guideline. It is still unclear what intensity, frequency and duration of PFMEs are most effective. Individual differences may require a different exercise regimen and when the condition of the pelvic floor muscles has recovered through exercising, a lower exercise frequency or intensity may be equally effective in maintaining the condition of the pelvic floor (Berghmans et al., 1998b; Bø, 1995b; Wells, 1990).

\section{Participants}

The study was approved by both the Medical Ethical Committee of Maastricht University and the Maastricht University Hospital and the Review Committee of the Registration Network Family Practices (RegistratieNet Huisartspraktijken, RNH) (Metsemakers et al., 1992). Participants were recruited from the RNH, which consists of 23 general practices with 55 general practitioners (GPs), covering a population of 
around 100,000 persons, whose data on relevant chronic health problems and demographic variables are registered and continuously updated. Since many women do not seek medical treatment for UI (Shaw et al., 2001), community-dwelling women over 17 years old with at least one of the following risk factors for UI were selected: vaginal delivery, medical history of gynecological operations, asthma, arthritis, and obesity (Diokno et al., 1990; Milson et al., 1993; Snooks et al., 1990; Yarnell et al., 1982). Selected women received a short questionnaire through their GP. The questionnaire consisted of items on risk factors for UI, continence status, quality of life according to continence status and use of health care resources for UI. Women reporting symptoms of stress, urge or mixed UI were also asked if they were interested in individual PFME therapy and participation in a behavioral intervention study. This procedure was conducted twice; once in 1995 in a study on the prevalence of UI among 2914 women with risk factors for this condition (Weijts et al., unpublished data), and once in 1997 among 1341 women when the present behavioral intervention study was set up. In 1995, 320 women, when asked, showed an interest in PFME therapy and 198 of them were still willing to do so in 1997. Of the second recruitment, 166 women were willing to participate, and a poster recruitment campaign in the participating general practices yielded another 12 women. Thus, a total of 376 women were interested in participation.

From January to October 1998, these 376 women received a letter from the researcher explaining that participation involved engaging in individual PFME therapy with a female physiotherapist and completing four questionnaires and diaries. The letter further instructed women to visit their GPs. After this letter, 180 women were actually willing to participate. The primary reasons given for refusal to participate by initially interested women were: symptoms of UI were minor or women were not sufficiently bothered by them to start PFME therapy (37\%), having other more severe health problems (14\%), having done PFME therapy or having been operated upon for UI $(12 \%)$, being too busy with work and/or care activities $(10 \%)$, no longer having symptoms of UI (5\%). Less frequently mentioned reasons were no interest in participation in a study, being afraid of vaginal palpation by the GP, feeling ashamed, being satisfied with the use of protective garments or medication, feeling too old to engage in PFME therapy, having no means of transport to the physiotherapist, or loss of mental capacity. Fifteen women had moved or died between the prevalence study and the start of the behavioral intervention study. 
In total, 13 general practices with 36 GPs were involved in the selection of participants. Inclusion criteria were: ability to complete questionnaires, full understanding of the Dutch language and completion of the consent form. Excluded from the study were women (total $n=47$ ) without symptoms of stress, urge or mixed UI $(n=11)$ based on their anamneses (Lagro-Janssen et al., 1995), women suffering from neurological conditions such as MS, CVA and spina bifida or suffering from venereal disease $(n=5)$, women with viral infections $(n=4)$, women using medication for UI or using medication that enhances/influences UI $(n=1)$, women who were pregnant or within three months after delivery or women who had been operated upon for UI $(n=20)$, and women with physical impairments making PFME therapy impossible $(n=6)$. The GP also evaluated the presence of prolapse and vaginal atrophy, and the ability to voluntarily contract the pelvic floor muscles through vaginal palpation. Women with urinary tract infection were treated and included when cured.

\section{Physiotherapists}

We recruited 28 female physiotherapists specialized in the field of PFME therapy with an outpatient physiotherapy practice in the residential area of the participating general practices. Mean age of the therapists was 40.8 years (SD 8.9; range 24-63). They had been in practice for on average 17.5 years (SD 9.1; range 1-39), and had been specialized in PFME therapy for on average 6.4 years (SD 5.6; range 1-25). Mean working hours per week of these physiotherapists amounted 27.4 (SD 9.7; range 1245). Yearly, they treated an average of 17 (SD 11; range 4-50) women with PFME therapy.

\section{Design and procedures}

A longitudinal randomized controlled trial with three intervention and one control condition was conducted. The control condition consisted of individual physiotherapeutic PFME therapy alone, representing usual care. The intervention conditions consisted of PFME therapy supplemented with one of the three health education interventions. The four conditions were randomly allocated to the physiotherapists. However, due to a shortage of therapists and to minimize the chance of contamination, the control condition and $\mathrm{Ri}$ were allocated to the same 
physiotherapists. The 133 eligible women were randomized in one of the four conditions by the GP's assistants, who had been instructed by the researcher. A randomization form, containing codes for the four conditions and for the physiotherapists serving the residential area of a particular general practice, facilitated the procedure and ensured that every physiotherapist would treat about the same number of participants, and that conditions would be equally filled. In this way, both the GPs and their assistants, as well as the participants were blinded for intervention groups. Physiotherapists could not be blinded for the intervention. For practical reasons only, the researcher could not be blinded either.

The use of the standardized protocol checklist during PFME therapy was instructed to all physiotherapists by the researcher, and each therapist was asked to fill out a treatment form for every patient. These forms consisted of the participant's name and date of birth, the diagnosis of the GP on the referral letter and of herself, and a table to evaluate treatment goals. For each experimental condition, the standardized protocol checklist was supplemented with instructions on how and when to use the health education intervention during treatment. This was instructed separately to the group of physiotherapists of each condition by the researcher. The Ri and R-SHGi had to be given to the women at the onset of therapy. The instruction for the guide was, whenever a woman would like to do so, to read about what she had learned during the sessions at home, to use it as a diary by writing down the homework exercise advice and/or to use it as a reminder during and after therapy. The R-SHG-Ci intervention was used in a similar way as the R-SHGi, with the difference that the instruction was more directive. Physiotherapists had to instruct women to read pages $\mathrm{x}$ to $\mathrm{y}$ of the guide that corresponded with the content of that particular therapy session. Furthermore, these physiotherapists had been instructed by the researcher to follow a counseling scheme with oral instructions corresponding to the design of the guide and to the iterative phases of the physiotherapeutic treatment plan (Verhulst et al., 1994). This counseling process was supposed to structurally guide patients from the awareness of the need for adherence, to taking up adherence behavior after relapses by providing oral feedback and reinforcement (Prochaska et al., 1992 and 1994).

\section{Questionnaire and diary}

All data were collected with self-administered questionnaires and 7-day diaries before (pretest), immediately after (posttest), and at 3 and 12 months after therapy. Women 
received the pretest survey from the GP's assistants before therapy and the posttest survey from the physiotherapists after the final PFME therapy session. The 3- and 12month follow-up surveys were mailed to the participants. When women did not return the surveys within three weeks after sending, they were phoned and asked to complete the surveys and return them as soon as possible in the prepraid envelope. Reasons for the delay and other aspects related to PFME therapy discussed in the call, were recorded.

The questionnaire was pilot-tested for readability, comprehensibility and credibility among 25 women doing PFME therapy. Screening of the subscales of the questionnaire with factor analysis using principal component analysis confirmed that all subscales constituted a coherent set of items. The reliability of the subscales was checked with Cronbach's alphas, using the standardized item $\alpha$ values (Tabachnick and Fidell, 1996). The proximal ASE and the distal variables addressing various aspects of adherence behavior, were translated into measurable concepts. Available scales were used as well. The assessment of adherence behavior and UI symptoms with the diary were both validated with several items in the questionnaire. The content of the questionnaire and diary is described below.

\section{Adherence behavior}

The 7-day diary assessed adherence in terms of number of days per week women had followed the behavioral advice of the physiotherapist at posttest and follow-ups. Three items on adherence behavior in the questionnaire were used as validation for the diary assessment (Kerssens et al., 1996), that all correlated highly and significantly $\left(\boldsymbol{r}_{\mathbf{d}}=.388\right.$ $-.632, \mathrm{p}<.001$ ) with the diary item. These three items assessed how often women had adhered to the behavioral advice the day and the week before, and a self-evaluation score for adherence on a scale from 1 to 10 .

\section{Intention and proximal variables}

Intention. The items 'Do you intend to follow the behavioral advice of your physiotherapist?' and 'Do you intend to exercise every day?' measured intention on seven-point scales (certainly not - yes, certainly; 1-7). The two items were summed to form one scale of intention $(\mathrm{r}=.56)$.

Attitude. Two scales were developed for attitude. The pros scale ( 8 items) referred to what women expected to gain by adhering to PFME therapy. These expectations were assessed on seven-point scales. For example: 'If I follow the behavioral advice, I can do my daily activities much better - much worse' $(7-1 ; \alpha=.83)$. The cons scale (5 
items) referred to the negative consequences of adherence to PFME therapy and was assessed on five-point scales. For example: 'If I follow the behavioral advice, I will become very tired - I don't know - not tired' $(5-1 ; \alpha=.55)$.

Social influence was measured with the concepts of perceived social norms, modeling, social support and social pressure. Perceived social norms were measured by six items on seven-point scales. For example: 'My friends think I should follow the therapist's advice'; 'I totally agree - I totally disagree' (7-1). The items were summed to form one sumscore (scale 7-42). Modeling was assessed by one question asking the respondent how many persons out of 10 categories (mother, sister, neighbor etc.) she knew who also had PFME therapy. The score ranged from 0 to 10 . Similarly, two items assessing social support asked with whom women (yes - no; 0 - 1) discussed their incontinence and PFME therapy $(r=.78)$. The two items were summed to form one index score $(0$ 35). Social pressure was measured by one item, asking 'Has anyone ever put pressure on you not to adhere to PFME therapy?'. Because only two respondents had ever experienced any social pressure, this item was excluded from further analysis.

Self-efficacy expectations were measured as both 'difficulties' and 'abilities' on sevenpoint scales (Bandura, 1997). In the 'abilities' scale (9 items), women's expectations of their ability to follow the behavioral advice regardless of situational demands, were measured. For example: 'I certainly can - cannot follow the behavioral advice when I am very busy' (7-1; $\alpha=.78)$. The 'difficulties' scale ( 9 items) referred to women's expectations of their skills regarding adherence to PFME therapy: 'Reminding myself every day to follow the behavioral advice is very easy - very difficult' $(7-1 ; \alpha=.87)$.

\section{Distal variables}

UI symptoms and perceived severity. The 7-day diary assessed weekly frequency of wet episodes (Nygaard and Holcomb, 2000; Wyman et al., 1988). In the questionnaire, these three aspects were also assessed on the basis of the PRAFAB score (Vierhout, 1990), for validation of the diary assessment. These three items correlated significantly with the diary assessment $\left(\mathrm{r}_{\mathrm{g}}: .551-.766, \mathrm{p}<.001\right)$. Another three items in the questionnaire measured the duration of symptoms, the type of UI and daily voiding frequency. Symptom distress was assessed with the Incontinence Quality of Life scale (IQOL, scale 22-110; Wagner et al., 1996; 22 items; $\alpha=.92$ ), and symptom impact with the Incontinence Impact Questionnaire (IIQ-7, scale 0-15; Uebersax et al., 1995; 7 items; $\alpha=.79$ ). Higher IQOL scores indicate a better quality of life in relation to incontinence symptoms, whereas higher IIQ-7 scores indicate a larger impact of symptoms on daily life. 
Self-esteem and body esteem. Self-esteem was measured using 12 items of one sevenpoint subscale of Bekker's Autonomy Questionnaire (Bekker, 1993). The subscale consisted of statements such as 'I usually know quite well what I like best'; totally agree - totally disagree $(7-1 ; \alpha=.79)$. For the concept of body esteem, no existing scale suitable for women with UI was available, so a scale was developed based on the interviews and literature (Franzoi, 1994; Franzoi and Shields, 1984). A pilot-test was conducted among 15 male and 15 female university staff. Body esteem was assessed by 13 items on seven-point scales using statements like I feel ashamed about my body'; totally agree - totally disagree $(1-7 ; \alpha=.84)$.

Sexual experiences were assessed by asking: 'Do you have any experience of sexual harassment, rape or incest before the age of $18^{\prime}$ and a similar question focussing on the period after the age of 18. Sex education was assessed with two seperate items: 'Did you have sex education at school', and, 'Have you been raised with the image that women may enjoy sex?'

Subjective general health was assessed in three ways. The abbreviated 'Inventory of Subjective Health' (VOEG; Sikkel, 1980) assessed chronic physical complaints. The 13 items were summed to form one index score (0-13). Two items of the Dutch National Health Survey assessed women's perception of their health on a five-point scale from very bad to very good (1-5), and which chronic conditions they had other than UI (index, 0-26) (Van den Berg, 1992).

Social desirability was assessed using the Social Desirability scale (Visser and Breemhaar, 1989; 10 items). Items such as 'If I make a mistake, I will always be prepared to admit it', were measured on a VAS scale (true --- false, 0-10), and summed to form a scale ranging from 0 to $100(\alpha=.63)$.

Sociodemographic variables were assessed with questions about age, education level, insurance status, having a partner, number of children, and hours per week spent on (paid) labor, housekeeping, care for relatives or other persons, physical and other leisure activities.

\section{Level of implementation of the health education interventions}

To assess the extent to which the health education program was implemented, all physiotherapists and women in the experimental conditions were asked to fill-out an additional questionnaire. For the women, this questionnaire was included in the posttest and consisted of 15 items that assessed whether women had read and used the 
health education materials and to what extent they had used the sticker reminders. Implementation of the oral counseling intervention was assessed among women in the R-SHG-Ci and among all physiotherapists with 13 items. These thirteen items assessed whether physiotherapists had discussed, for example, what treatment goals a woman wanted to reach in order to set small subgoals, pros and cons of continuous adherence behavior or possibilities of taking up adherence behavior after relapses. Experiences with the implementation of the protocol checklist in PFME therapy in general, were assessed among all physiotherapists.

\section{Analyses}

Data were analyzed using SPSS version 9.0, and procedures for data screening, randomization check and selectivity of dropouts, were applied according to Tabachnick and Fidell (1996). Descriptive statistics were used to describe sociodemographic and other distal variables. Two-tailed tests on Spearman correlation coefficient (continuous and ordinal variables) and Mann-Whitney tests (dichotomous variables) were used to check the relation of determinants with adherence behavior.

Both longitudinal and cross-sectional sequential multiple linear regression analyses were applied to determine significant predictors of adherence to PFME therapy, incorporating only those determinants that significantly correlated with adherence at least at one of the three measurements, but not with each other. To determine whether the health education program had any effect, it was entered in the first block. The second block contained external variables; the third block only those proximal variables that significantly correlated with adherence; and the fourth block contained the intention to adhere to PFME therapy; and in the longitudinal analyses for follow-up adherence behavior, the fifth block contained adherence at posttest.

Univariate one-way analysis of covariance tests were used to compare adherence behavior between conditions at posttest, 3- and 12-month follow-up, and multivariate one-way analysis of covariance tests were used to compare changescores of determinants between conditions, both after adjustment for pretest weekly frequency of wet episodes. Repeated measurement analyses of variance were used to detect changes over time with post hoc paired samples t-test with Holm's bonferroni correction $(\alpha=$ $.05 / \mathrm{n}$ tests). Subgroup analyses for diagnostic groups were performed using the diagnosis of the physiotherapist as that determined therapy content. 


\section{RESULTS}

\section{Non-response analysis}

Four women declined to complete the pretest questionnaire and never started PFME therapy. Three of them had medical problems (symphiolysis, rheumatic complaints, severe tooth pain), were unable to do PFME therapy and should have been excluded before by the GP. One woman claimed that she wanted to complete the questionnaire and start therapy, but never did, regardless of reminder calls.

Loss to follow-up numbers were not significantly different between intervention groups; control 3/32, Ri 7/29, R-SHGi 9/34, R-SHG-Ci 7/34. Twenty-six women ( $20 \%$ of 129) withdrew either immediately after randomization $(n=10)$, during the treatment period $(n=4)$, or during the follow-up measurements $(n=12)$. Reasons or combinations of reasons for withdrawal included personal illness $(\mathrm{n}=12)$ and/or an ill relative $(n=3)$, stressful life or busy schedule $(n=8)$, transportation problems $(n=2)$, symptoms not improved $(n=2)$, lack of interest $(n=7)$. One women died after the 3 month follow-up and one woman found out that she went to the toilet only three times a day through completing the pretest diary. Knowing this, she started to toilet more often and by doing so, eliminated the UI symptoms and no longer needed PFME therapy.

The 26 women lost to follow-up differed from the women who completed the study on two variables at pretest. The women lost to follow-up had more frequent $(\underline{U}=978.5, \mathbb{R}$ $=0.029)$ and more severe $(\underline{U}=1036.5, \underline{\mathrm{Q}}=0.045)$ wet episodes as measured with the questionnaire. All analyses, except intention to treat (last observation carried forward), were performed with the 103 women who completed the 12-month follow up measurements. Of those 103 women, the posttest of 15 women and the 3-month follow-up of two women were not returned due to unknown reasons.

\section{Characteristics of participants}

Sociodemographic characteristics and other distal variables at pretest of the 129 remaining participants are presented in Table 1 . The youngest woman was 27 , the oldest 82 . Most women had a partner and children, and one in three women still had children living at home. Of the 45 women with a paid job, $40 \%$ worked more than three days per week. Taking care of spouse, children and/or important others, 
Table 1. Characteristics of participants, sociodemographic and other distal variables, $N=129$

\begin{tabular}{llll} 
Variables & $\begin{array}{l}\text { Number of } \\
\text { women }\end{array}$ & $\begin{array}{l}\text { Percentage of } \\
\text { population }\end{array}$ & Mean (SD) \\
\hline
\end{tabular}

Age

Having a partner

107

$82.9 \%$

Having children

118

$91.5 \%$

Number of children

Having a paid job

45

$34.9 \%$

Hours of paid labor per week

$55.6(10.9)$

Hours of care' per week

Hours of activities ${ }^{2}$ per week

Hours of sport per week

Education Level $(n=127)$

$$
\begin{aligned}
& \text { Low }^{3} \\
& \text { Medium } \\
& \text { Highs }
\end{aligned}
$$

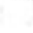

Had sex education at school

Social Health Insurance

Recruitment phase 1995

Recruitment phase 1998

$\begin{array}{ll}84 & 65.1 \% \\ 58 & 45.0 \% \\ 96 & 75.6 \% \\ 16 & 12.4 \% \\ 15 & 11.8 \% \\ 43 & 33.3 \% \\ 98 & 76.0 \% \\ 69 & 53.5 \% \\ 60 & 46.5 \%\end{array}$

Body mass index

VOEG

General health status

Good
Moderate
Poor

Other chronic conditions ( 0 -26)

Sexual violence in youth

Sexual violence in adult life

Diagnosis of physiotherapist $(\mathrm{n}=99)$

Stress
Urge
Mixed
missing

Duration of symptoms $(\mathrm{n}=127)$
less than 2 years
2.7 years

over 7 years

Weekly frequency of wet episodes,

7-day diary $(n=125)$

IQOL $(n=128)$

IIQ-7

Self-esteem $(n=126)$

Body esteem $(n=126)$
$26.9(4.8)$

$7.2(3.2)$

$\begin{array}{lr}46 & 35.7 \% \\ 52 & 40.3 \% \\ 31 & 24.0 \% \\ & \\ 22 & 17.1 \% \\ 16 & 12.4 \% \\ & \\ 48 & 37.2 \% \\ 11 & 8.5 \% \\ 40 & 31.0 \% \\ 30 & 23.3 \% \\ & \\ 11 & 8.7 \% \\ 72 & 56.7 \% \\ 44 & 34.6 \%\end{array}$

$2.5(1.9)$

$7.2(6.7)$

$24.5(25.0)$

$2.2(2.7)$

$60.0(10.8)$

$62.2(12.0)$

1. Care activities involved hours per week spent on household and caring for (ill) important persons.

2. Activities involved hours per week spent on taking courses, or doing club or voluntary work.

3. Low education level: completed primary school and vocational school

4. Medium education level: completed secondary (vocational) school

5. High education level: completed the highest level of secondary (vocational) school or university 
combined with household activities was taking women on average 27 hours per week. Forty-five percent of the women was involved in sports activities and $65 \%$ was taking courses, or engaged in leisure activities such as voluntary or club work. More women had a low education level as compared with the general Dutch population (www.cbs.nl, 2001), and two-third of the women had sex education at school. Most women were insured under the Social Health Insurance scheme.

Mean body mass index was 26.9 (SD 4.8 ), which is slightly above normal. Twice as many physical complaints (VOEG) were reported as found among middle-aged

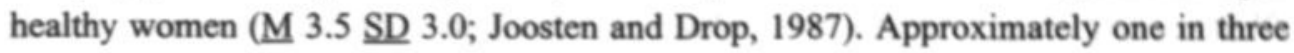
women evaluated their own health status as good, one in four as not so good, two in five as moderate, and four in five women mentioned having other chronic diseases. Experiences of sexual violence in the youth and in adult life were reported by 22 $(17.1 \%)$ and $16(12.4 \%)$ women, respectively.

Physiotherapists diagnosed $37.2 \%$ of the women with stress, $31.0 \%$ with mixed and $8.5 \%$ of the women with urge incontinence, but unfortunately missed to fill-out the diagnosis of the other women $(n=30)$. Most women reported having symptoms of moderate severity for several years. Mean weekly frequency of wet episodes was 24.5 (SD 25.0). Women occasionally worried about their incontinence symptoms (IQOL) and wet episodes had some negative impact on daily life activities (IIQ-7). On average, women had a relatively high self-esteem and a slightly positive body esteem.

Data on proximal variables are presented in Table 2. Intention to adhere to PFME therapy was high at the onset of therapy. Women had slightly positive outcome expectations, but also had some negative outcome expectations towards following the behavioral advice. Many women were convinced that significant others would want them to follow the advice of the physiotherapist, but they knew few role models. Incontinence problems and PFME therapy were discussed with on average two to three people, mostly spouse, daughters, and female family members or friends. Women were neutral about their self-efficacy expectations of skills regarding adherence to PFME therapy (difficulties), and had slightly positive self-efficacy expectations about following the behavioral advice under various situations, such as during weekends or when busy (abilities). 
Table 2. Construct (number of women completing the items), number of items, mean scale scores with standard deviation (SD), item range and mean item scores at pretest of intention and proximal variables.

\begin{tabular}{lllll}
\hline $\begin{array}{l}\text { Construct } \\
\text { (number of women) }\end{array}$ & $\begin{array}{l}\text { number of } \\
\text { items }\end{array}$ & $\begin{array}{l}\text { Mean scale score } \\
\text { (SD) pretest }\end{array}$ & $\begin{array}{l}\text { Item range } \\
\text { (SD }\end{array}$ & $\begin{array}{l}\text { Mean item } \\
\text { score pretest }\end{array}$ \\
\hline
\end{tabular}

$\begin{array}{lllll}\text { Intention }(\mathrm{n}=124) & 2 & 12.8(1.5) & 1-7 & 6.4\end{array}$

Attitude

- pros $(n=127)$

- cons $(n=128)$

8
5

$41.4(5.3)$

$1-7$

4.6

Social Influence

- Social norms $(n=128)$

- Modeling $(n=127)$

$11.0(2.6)$

$1-5$

2.8

- Social support $(n=125)$

$32.6(5.0)$

$1-7$

5.4

$0.3(0.5)$

$0-10$

0.3

Self-efficacy expectations

- abilities $(\mathrm{n}=126)$

- difficulties $(\mathrm{n}=125)$

$5.1(3.5)$

$0-17$

2.6

9

40.7 (6.4)

$1-7$

4.5

$35.0(9.0)$

$1-7$

Significant differences between conditions at pretest were as follows: women in the control condition used heavier pads than women in the R-SHGi $(\underline{H}(3)=9.143, \underline{p}=$ $.027)$, and women in the $\mathrm{Ri}$ experienced more symptom distress than women in the $\mathrm{R}$ SHG-Ci $(F(3.124)=3.079, \mathrm{D}=.030)$ and more symptom impact than women in the RSHGi $(\mathrm{E}(3,124)=3.105, \mathrm{p}=.029)$. Further, fewer women in the control condition had sex education at school than women in the R-SHGi $(\underline{H}(3)=4.098, \underline{p}=.043)$. With $n=$ 129 , no differences were found among groups on any of the other variables at pretest. However, with $\mathrm{n}=103$, pretest scores of weekly frequency of wet episodes were significantly higher $(\underline{H}(3)=8.943, p=.030)$ in the control $(\underline{M} 27.6, \underline{S D} 21.9)$ condition than in the R-SHGi (M 12.8, $\underline{\mathrm{SD}}$ 12.4).

\section{Implementation of health education interventions}

The assessment of the level of implementation of the three interventions among women and physiotherapists revealed that both the reminder and the counseling intervention had not been implemented as planned. While the therapists thought that most women would use the stickers, two in five women of the Ri and the R-SHG-Ci actually used a few stickers, and only two women in the R-SHGi did so. Thus, the use of stickers and other reminders suited only some of the women, regardless of the 
condition to which they had been allocated. In addition, no relation was found between the use of stickers and adherence behavior.

The assessment of the level of implementation among physiotherapists after the intervention period had ended, revealed that all 13 key aspects of oral counseling had been implemented to the same extent by the physiotherapists in all conditions, although only the therapists in the R-SHG-Ci had actually been trained in applying the specific counseling scheme to provide structural oral feedback and reinforcement.

Thus, having had a self-help guide remained as the only distinction between the study groups. Therefore secondary analyses were conducted in which women in the two selfhelp guide groups ( $\mathrm{R}-\mathrm{SHGi}+\mathrm{R}-\mathrm{SHG}-\mathrm{Ci}$ ) were compared with women in the other two groups (Control condition $+\mathrm{Ri}$ ), to study the possible surplus value of the self-help guide on adherence behavior and determinants. To check for consistency of the results, women in the self-help guide groups were also compared with women in the control condition alone.

\section{Predictors of adherence behavior}

Overall, adherence behavior was high with women following the behavioral advice on average 6.2 days per week (SD 1.2) at posttest, 5.5 days per week (SD 2.0) at 3-months follow-up and on average 4.8 days per week (SD 2.4) one year after therapy. Sequential multiple linear regression analyses were conducted to identify which determinants predicted adherence behavior at posttest (short-term), 3- and 12-month follow-up (long-term), when taking into account the order of input as determined by the theory of the ASE model. First assumptions were checked. Adherence behavior was slightly negatively skewed without transformation, but positively with it; thus it was not transformed. Weekly frequency of wet episodes was positively skewed, but analyses with transformed data revealed the same results, therefore results are presented with untransformed data. With the use of a $p<.001$ criterion for Mahalanobis distance, several outliers among the independent variables were identified. However, analyses with and without these outliers revealed the same results, and no differences were found between outliers and the other group members between these independent variables; thus these women were not excluded from the analyses. Five women were significant univariate outliers on the dependent variable of adherence behavior, having too large standardized residuals, and were left out of the regression analyses because they significantly altered results. Possible interaction 
terms of independent variables were checked and no significant interactions were found.

The analyses with the three dummies for the original intervention conditions revealed no significant contribution of the separate health education interventions on adherence behavior. Further, because the reminder and oral counseling had not been implemented as planned, one dummy for 'group with or without self-help guide' was entered in the first block. Of the proximal variables, positive outcome expectation, social norms and self-efficacy abilities were included in the model, as the other proximal variables were not significantly related to adherence behavior.

\section{Longitudinal analyses: predicting adherence behavior after therapy with pretest data} Longitudinal sequential regression analyses were used to reveal which determinants assessed at pretest predicted adherence behavior immediately after therapy, and three and twelve months later. Previous adherence behavior assessed at posttest was included in both regression analyses with dependent variable adherence at the followups. Results are presented in Table 3.

At posttest, the first analysis in this sequence indicated that the self-help guide did not account for a significant amount of variability in adherence, $\underline{\mathrm{R}^{2}}$ change $=.044, \mathrm{p}=$ .067. The second analysis revealed that none of the distal variables contributed to variability in adherence over and above the self-help guide, $\mathrm{R}^{2}$ change $=.103, \mathrm{p}=.084$. The third analysis indicated that of the proximal variables, both self-efficacy abilities and social norms significantly contributed to variability in adherence over and above the self-help guide and distal variables, $\underline{\mathrm{R}}^{2}$ change $=.124, \mathrm{p}=.013$. The fourth and final analysis indicated that the intention to adhere at pretest did not significantly account for variability in posttest adherence behavior over and above proximal and distal variables and the self-help guide, $\underline{\mathrm{R}}^{2}$ change $=.009, \underline{\mathrm{p}}=.368$. Thus, the self-help guide and social norms were left as significant predictors of posttest adherence behavior. These results suggest that women who perceived more social norms regarding adherence behavior, and women who were in the group without the self-help guide, followed the behavioral advice on more days per week than women who perceived fewer social norms and women in the group with the guide. Thus, intention did not mediate for the proximal and distal variables. This final model predicted $28 \%$ (adjusted 18\%) of the variance in short-term adherence behavior. 
Table 3. Three sequential longitudinal multiple linear regression analyses with adherence behavior at posttest, 3- and 12-month follow-up as dependent variables and the independent variables from pretest that significantly predicted adherence behavior; standardized $\beta$ coefficients, $P$ values and explained variance for final equations.

\begin{tabular}{|c|c|c|c|c|c|c|}
\hline \multirow[t]{2}{*}{ Independent variables } & \multicolumn{2}{|c|}{$\begin{array}{l}\text { Posttest, } \\
\mathrm{n}=77, \underline{\mathrm{R}^{2}=.28,} \\
\mathrm{R}^{2} \text { adjusted }=.18\end{array}$} & \multicolumn{2}{|c|}{$\begin{array}{l}\text { 3-month follow-up, } \\
\mathrm{n}=73, \mathrm{R}^{2}=.37 \\
\underline{\mathrm{R}^{2}} \text { adjusted }=.27\end{array}$} & \multicolumn{2}{|c|}{$\begin{array}{l}\text { 12-month follow-up, } \\
\mathrm{n}=75, \mathrm{R}^{2}=.50, \\
\mathrm{R}^{2} \text { adjusted }=.42\end{array}$} \\
\hline & & $\mathrm{p}$ & & p & & p \\
\hline Block 1 (condition): & & & & & & \\
\hline Self-help guide & -.226 & .049 & .131 & .249 & .057 & .570 \\
\hline Block 2 (distal): & & & & & & \\
\hline Weekly wet episodes & .201 & .093 & .089 & .449 & .276 & .010 \\
\hline Weekly hours of care & .081 & .488 & .130 & .258 & .044 & .663 \\
\hline Sex education at school & .211 & .053 & -.047 & .663 & -.458 & .000 \\
\hline $\begin{array}{l}\text { Negative sexual } \\
\text { experiences in adult life }\end{array}$ & .142 & .200 & -.071 & .513 & -.179 & .065 \\
\hline Block 3 (proximal): & & & & & & \\
\hline Attitude: pros & -.072 & .563 & -.003 & .979 & -.032 & .759 \\
\hline Social norms & .244 & .041 & .029 & .809 & -.006 & .955 \\
\hline Self-efficacy abilities & .211 & .081 & .319 & .009 & .188 & .074 \\
\hline Block 4: & & & & & & \\
\hline $\begin{array}{l}\text { Intention } \\
\text { or }\end{array}$ & .110 & .368 & .140 & .238 & -.095 & .368 \\
\hline $\begin{array}{l}\text { Adherence behavior at } \\
\text { posttest }\end{array}$ & & & .328 & .008 & .428 & .000 \\
\hline
\end{tabular}

Three months after therapy, the first analysis in this sequence indicated that the selfhelp guide did not account for a significant amount of variability in adherence, $\underline{R}^{2}$ change $=.001, \mathrm{p}=.842$. Also, in the second analysis none of the pretest measured distal variables contributed significantly to adherence variability over and above the self-help guide, $\mathrm{R}^{2}$ change $=.045, \mathrm{p}=.538$. The third analysis revealed that of the proximal variables, self-efficacy abilities accounted for a significant amount of variance in adherence, $\underline{\mathrm{R}}^{2}$ change $=.226, \mathrm{p}=.001$. The fourth analysis indicated that intention did not significantly account for variability in posttest adherence behavior over and above proximal and distal variables, $\mathrm{R}^{2}$ change $=.024, \mathrm{p}=.146$. The fifth and final analysis included posttest adherence behavior, which, together with self-efficacy abilities, significantly contributed to adherence variability over and above distal variables and the self-help guide, $\underline{\mathrm{R}}^{2}$ change $=.075, \mathrm{p}=.008$. This indicates that women who had positive self-efficacy expectations regarding their adherence behavior under various circumstances, and women who had high adherence levels at posttest, 
retained their high adherence levels three months after therapy. This final model predicted $37 \%$ (adjusted $27 \%$ ) of the variance in adherence behavior three months after therapy.

One year after therapy, the first analysis in this sequence revealed that the self-help guide did not significantly account for variability in adherence, $\underline{\mathrm{R}}^{2}$ change $=.049, \mathrm{p}=$ .057. The second analysis showed that of the distal variables, weekly frequency of wet episodes and sex education at school significantly contributed to variability in adherence, over and above the self-help guide, $\underline{\mathrm{R}}^{2}$ change $=.243, \underline{\mathrm{p}}<.001$. The third analysis with the proximal variables did not significantly account for the amount of variability in adherence over and above distal variables and the self-help guide, $\underline{\mathrm{R}}^{2}$ change $=.070, \mathrm{p}=.074$. The fourth analysis indicated that intention did not significantly account for variability in posttest adherence behavior over and above proximal and distal variables, $\mathrm{R}^{2}$ change $=.002, \mathrm{p}=.686$. The fifth and final analysis showed a significant contribution of posttest adherence behavior to variability in longterm adherence over and above proximal variables and the self-help guide, and a significant contribution of the distal variables of weekly frequency of wet episodes and sex education at school, $\underline{\mathrm{R}}^{2}$ change $=.133, \underline{\mathrm{p}}<.001$. These results suggest that women who had frequent wet episodes at the onset of therapy, and women who adhered well at posttest, continued to adhere well one year after therapy, and that women who had no sex education at school also had high long-term adherence scores. This final model predicted $50 \%$ (adjusted $42 \%$ ) of the variance in adherence behavior one year after therapy.

Several conclusions can be drawn from these longitudinal analyses. The group without the self-help guide had a higher adherence level at posttest than the group with the guide. And, women who perceived more social norms regarding adherence behavior at pretest had a higher adherence level at posttest, than women who were less influenced by social norms. Women who were convinced that they could follow the behavior advice of the physiotherapist before the start of therapy, had high adherence levels three months after therapy. Further, women who had frequent weekly wet episodes before therapy had higher adherence levels one year after therapy than women with fewer weekly losses. Women who had no sex education at school also had high adherence levels one year after therapy. Short-term adherence appeared to be an important predictor of long-term adherence behavior. 


\section{Cross-sectional analyses: predictors of adherence behavior per measurement}

Cross-sectional sequential regression analyses were employed to reveal which determinants predicted adherence behavior immediately after therapy, and three and twelve months later. Results are presented in Table 4. Sex education at school was included because of differences between conditions at pretest. Per analysis, the remaining variables were all measured at the same moment as the dependent variable adherence.

Table 4. Three sequential cross-sectional multiple linear regression analyses with adherence behavior at posttest, 3- and 12-month follow-up as dependent variables and the independent variables from the same measurements that significantly predicted adherence behavior; standardized $\beta$ coefficients, $P$ values and explained variance for final equations.

\begin{tabular}{|c|c|c|c|c|c|c|}
\hline \multirow[t]{2}{*}{$\begin{array}{l}\text { Independent variables } \\
\text { per measurement }\end{array}$} & \multicolumn{2}{|c|}{$\begin{array}{l}\text { Posttest, } \mathrm{n}=85, \\
\mathrm{n}=77, \mathrm{R}^{2}=.31 \\
\mathrm{R}^{2} \text { adjusted }=.22\end{array}$} & \multicolumn{2}{|c|}{$\begin{array}{l}\text { 3-month follow-up, } \\
\mathrm{n}=75, \mathrm{R}^{2}=.43 \\
\mathrm{R}^{2} \text { adjusted }=.36\end{array}$} & \multicolumn{2}{|c|}{$\begin{array}{l}\text { 12-month follow-up, } \\
\mathrm{n}=77, \mathrm{R}^{2}=.55, \\
\mathrm{R}^{2} \text { adjusted }=.50\end{array}$} \\
\hline & $\beta$ & $\mathrm{p}$ & $\beta$ & p & & p \\
\hline $\begin{array}{l}\text { Block } 1 \text { (condition): } \\
\text { Self-help guide }\end{array}$ & -.114 & .316 & .185 & .078 & .075 & .405 \\
\hline Block 2 (distal): & & & & & & \\
\hline Weekly wet episodes & -.014 & .890 & -.106 & .310 & .247 & .007 \\
\hline Weekly hours of care & .077 & .484 & .180 & .083 & .125 & .154 \\
\hline Sex education at school & .154 & .144 & -.087 & .367 & -.210 & .017 \\
\hline Block 3 (proximal): & & & & & & \\
\hline Attitude: pros & .065 & .620 & -.048 & .696 & -.017 & .874 \\
\hline Social norms & .034 & .781 & .100 & .369 & .058 & .583 \\
\hline Self-efficacy abilities & .211 & .174 & .235 & .066 & .269 & .010 \\
\hline $\begin{array}{l}\text { Block 4: } \\
\text { Intention }\end{array}$ & .279 & .035 & .495 & .000 & .423 & .000 \\
\hline
\end{tabular}

At posttest, the first analysis indicated that the self-help guide did not account for a significant amount of variability in adherence, $\underline{R}^{2}$ change $=.044, \mathrm{R}=.068$. The second analysis revealed that none of the distal variables contributed to variability in adherence over and above the self-help guide, $\underline{R}^{2}$ change $=.037 \mathrm{R}=.410$. The third analysis indicated that of the proximal variables, self-efficacy abilities significantly contributed to variability in adherence over and above distal variables and the self-help guide, $\mathrm{R}^{2}$ change $=.176, \mathrm{p}=.002$. The fourth and final analysis indicated that the intention to adhere significantly accounted for variability in adherence over and above 
proximal and distal variables and the self-help guide, $\underline{\mathrm{R}}^{2}$ change $=.047, \underline{\mathrm{p}}=.035$. This means that intention mediated the influence of self-efficacy abilities and the other proximal and distal variables. This final model explained $31 \%$ (adjusted $22 \%$ ) of variance in adherence behavior at posttest. Thus, women who intented to continue to follow the behavioral advice at posttest, also had high posttest adherence levels.

At 3-months follow-up, the first analysis indicated that the self-help guide did not account for a significant amount of variability in adherence, $\underline{\mathrm{R}}^{2}$ change $=.000, \mathrm{p}=$ .911. Also, none of the distal variables in the second analysis contributed significantly to adherence variability over and above the self-help guide, $\underline{\mathrm{R}}^{2}$ change $=.041, \mathrm{p}=.405$. The third analysis revealed that of the proximal variables, self-efficacy abilities accounted for a significant amount of variance in adherence, $\mathrm{R}^{2}$ change $=.235, \mathrm{p}<$ .001 . The fourth and final analysis showed that intention significantly contributed to adherence variability over and above proximal and distal variables, and the self-help guide, $\underline{\mathrm{R}}^{2}$ change $=.155, \mathrm{p}<.001$. This indicates that women who declared at 3 -month follow-up, to have a positive intention to adhere to PFME therapy recommendations, also had high adherence levels at that moment, and again intention mediated the influence of self-efficacy and the other variables in the equation. This final model predicted $43 \%$ (adjusted $36 \%$ ) of the variance in adherence behavior three months after therapy.

One year after therapy, the first analysis revealed that the self-help guide did not significantly account for variability in adherence, $\underline{\mathrm{R}}^{2}$ change $=.041, \mathrm{p}=.079$. The second analysis showed that of the distal variables, weekly frequency of wet episodes at one year after therapy and sex education at school significantly contributed to variability in adherence, over and above the self-help guide, $\mathrm{R}^{2}$ change $=.161, \underline{p}=.004$. The third analysis revealed that of the proximal variables, self-efficacy abilities significantly accounted for the amount of over and above the self-help guide, together with weekly frequency of wet episodes and sex education at school, $\underline{R}^{2}$ change $=.236$, $\mathrm{p}<.001$. The fourth and final analysis showed a significant contribution of the intention to adhere, of self-efficacy abilities, and of weekly frequency of wet episodes and sex education at school to adherence variability, over and above the self-help guide, $\mathrm{R}^{2}$ change $=.111, \mathrm{p}<.001$. These results suggest that women who had no sex education at school, women who still had frequent wet episodes at 12-month followup, and women who had positive self-efficacy expectations and intended to adhere 
well at that moment, had high adhere levels one year after therapy. This final model predicted $55 \%$ (adjusted $50 \%$ ) of the variance in adherence behavior twelve months after therapy.

Several conclusions can be drawn from these cross-sectional analyses. Whether women did or did not have the self-help guide was of no influence to adherence behavior. Positive intention was the most important predictor of high adherence levels at posttest and three months after therapy, mediating the influence of the proximal and distal variables. But one year after therapy, intention did not fully mediate the influence of other variables, and high long-term adherence levels were also significantly predicted by having had sex education at school, having frequent wet episodes and by positive expectations of the ability to follow the behavioral advice.

\section{Influence of the health education program on adherence and determinants}

As noted in the previous paragraph, adherence behavior was very high. One year after therapy, $67 \%$ of the women followed the behavioral advice four to seven days per week. The program component self-help guide appeared a significant predictor for posttest adherence behavior and nearly for adherence behavior at 3-months follow-up. Therefore, analyses were performed to check for differences in adherence behavior between intervention conditions, after adjustment for pretest frequency of wet episodes per week. No significant differences were found among the four study conditions in the number of days per week women had followed the behavioral advice at posttest, 3and 12-month follow-up. The secondary analyses did not change this finding. Shortand long-term adherence behavior, or the change in adherence between posttest and follow-ups was not different for the group with the self-help guide compared to the group without the self-help guide.

At posttest, women with stress or mixed UI had followed the behavioral advice more days per week ( $\mathrm{six}$ ) than women with urge UI (five) $(\mathrm{E}(2,72)=3.690, \mathrm{R}=.030)$, whereas no differences were found between diagnostic groups in adherence behavior at 3- and 12-month follow-up. Adherence behavior for each diagnostic group was also compared between intervention conditions, again after adjustment for pretest frequency of wet episodes. Adherence behavior at posttest, 3- and 12-month follow-up, did not differ between conditions for women with either, stress, urge or mixed UI.

Table 5 presents change in intention, proximal and distal variables between pretest and 12-month follow-up within the group with and the group without the self-help guide. 
Table 5. Change in intention, proximal and distal variables between pretest (t1) and 12 -month follow-up (t4) within groups, and comparison between the group without and the group with the self-help guide based on the changescores (t1-t4) of variables after adjustment for pretest weekly frequency of wet episodes $(n=103)$.

\begin{tabular}{|c|c|c|c|c|c|c|c|}
\hline \multirow[t]{2}{*}{ Variables } & \multicolumn{3}{|c|}{$\begin{array}{l}\text { group without self-help guide } \\
\mathrm{n}=51 \text {, mean (SD), change }\end{array}$} & \multicolumn{3}{|c|}{$\begin{array}{l}\text { group with self-help guide } \\
n=52 \text {, mean (SD), change }\end{array}$} & \multirow{2}{*}{$\begin{array}{r}\begin{array}{l}\text { between } \\
\text { groups }\end{array} \\
\mathrm{p}^{2} \\
\end{array}$} \\
\hline & $\mathrm{t} 1$ & 14 & $\mathrm{R}^{1}$ & $\mathrm{t} 1$ & $\mathrm{t} 4$ & $\mathbf{p}^{\prime}$ & \\
\hline Intention & $12.8(1.6)$ & $9.8(3.7)$ & $<.001$ & $12.8(1.3)$ & $8.0(3.3)$ & $<.001$ & ns \\
\hline \multicolumn{8}{|l|}{ Attitude } \\
\hline - pros & $41.0(5.9)$ & $42.3(6.1)$ & ns & $41.7(5.0)$ & $41.1(5.5)$ & ns & ns \\
\hline - cons & $11.9(2.5)$ & $10.4(3.4)$ & .008 & $11.8(2.2)$ & $10.6(3.1)$ & ns & ns \\
\hline \multicolumn{8}{|l|}{ Social Influence } \\
\hline - Social norms & $32.3(4.8)$ & $31.0(5.8)$ & ns & $32.7(5.1)$ & $31.2(5.5)$ & ns & ns \\
\hline - Modeling & $0.3(0.6)$ & $0.6(0.7)$ & ns & $0.3(0.5)$ & $0.4(0.6)$ & ns & ns \\
\hline - Social support & $5.2(3.9)$ & $6.0(5.7)$ & ns & $5.0(3.1)$ & $4.9(4.2)$ & ns & ns \\
\hline \multicolumn{8}{|l|}{$\begin{array}{l}\text { Self-efficacy } \\
\text { expectations }\end{array}$} \\
\hline - abilities & $41.5(6.7)$ & $39.5(10.3)$ & ns & $40.4(6.3)$ & $36.9(8.6)$ & .007 & ns \\
\hline - difficulties & $36.0(9.7)$ & $37.6(11.0)$ & ns & $34.7(8.1)$ & 35.1 ( 9.5$)$ & ns & ns \\
\hline IQOL & $80.5(15.7)$ & $92.8(14.4)$ & $<.001$ & $88.5(11.2)$ & $94.6(12.5)$ & $<.001$ & ns \\
\hline IIQ-7 & $2.6(3.1)$ & $1.3(2.8)$ & $<.001$ & $1.4(1.5)$ & $0.4(0.9)$ & $<.001$ & ns \\
\hline Self-esteem & $58.4(11.4)$ & $61.1(8.9)$ & ns & $60.3(10.6)$ & $61.5(10.5)$ & ns & ns \\
\hline Body esteem & $60.0(13.2)$ & $64.2(14.8)$ & .008 & $63.6(10.8)$ & $66.1(10.5)$ & ns & ns \\
\hline VOEG & $8.0(3.3)$ & $7.3(3.9)$ & ns & $6.9(3.1)$ & $5.8(3.3)$ & .001 & ns \\
\hline general health & $2.9(0.8)$ & $2.8(1.1)$ & ns & $3.4(0.9)$ & $3.4(0.9)$ & ns & ns \\
\hline
\end{tabular}

1. One way Repeated Measures ANOVA with post hoc paired samples t-test with Holm's bonferroni correction for multiple comparisons.

2. Multivariate ANCOVA for comparison of changescores of variables between group with and group without self-help guide after adjustment for pretest weekly frequency of wet episodes. Intention and proximal variables in one model $(\mathrm{F}(8,79)=1.505, \mathrm{p}=.169)$, distal variables in another model $(\mathrm{F}(6,89)=1.035, \mathrm{p}=.408)$; Holms' bonferroni correction. 
In both groups, the intention to adhere to PFME therapy had significantly decreased, and quality of life in relation to symptoms of UI had significantly improved (IQOL, IIQ-7). Further, in the group without the self-help guide, women had less negative outcome expectations and a more positive body esteem one year after therapy. In the group with the self-help guide, women's expectations about their ability to follow the behavioral advice decreased, and women had less physical impairments (VOEG) one year after therapy. The rest of the variables remained stable between pretest and one year after therapy.

Table 5 also shows the comparison between the group without and the group with the self-help guide based on the changescores of intention, proximal and distal variables between pretest and 12-month follow-up, after adjustment for pretest weekly frequency of wet episodes. No significant differences between groups were found, thus the selfhelp guide had no additional influence on these variables.

\section{DISCUSSION}

The first aim of this study was to assess predictors of long-term adherence to PFME therapy among women with UI. Total explained variance of long-term adherence behavior one year after PFME therapy was fairly high. The prediction of long-term adherence with pretest data yielded an adjusted $\mathrm{R}^{2}$ of .42 , while cross-sectional predictors assessed at one year after therapy yielded an adjusted $\mathrm{R}^{2}$ of .50. To compare, in a review of Godin and Kok (1996), the prediction of various health related behaviors by concepts of the TPB, yielded an average $R^{2}$ of .34 , thus adjusted $R^{2}$ would be lower. The reason why we used adjusted $\mathrm{R}^{2}$ was that the number of independent variables included in the regression analyses was relatively large compared to the number of observations, and adjusted $\mathrm{R}^{2}$ corrects for this situation. Clearly, other concepts not measured in this study, play a role in the prediction of the variance in adherence behavior, warranting further research on this topic.

Derived from the cross-sectional analysis, intention to adhere to PFME therapy recommendations was the most important predictor of adherence behavior at posttest and three and twelve months after therapy. Intention totally mediated the influence of the proximal and distal variables at posttest and three months follow-up, as found by others (Godin and Kok, 1996), but partly at one year after therapy. However, an important finding was that, in contrast to the theoretical assumption of the ASE model (De Vries et al., 1988), the longitudinal and strongest analysis revealed that intention at 
pretest did not significantly predicted adherence at posttest and follow-ups. An explanation might be that most women had a very positive intention to adhere to PFME therapy before the therapy had started, which is often the case with desirable behavior. As a consequence, intention data were positively skewed and standard deviations were very small. This situation statistically hinders a relation between adherence and intention. As expected, short-term adherence significantly predicted long-term adherence, and probably mediated the influence of intention. Ajzen (2001) states that [quote] 'although the TPB from which the ASE model is derived, generally assumes reasoned process underlying attitudes and action, there is in contrast an unmediated link between prior and later behavior. This implies habituation in a process that bypass intentions.' In two studies, past behavior was also found to be a strong predictor of subsequent behavior, besides proximal ASE variables and intention. One study analyzed screening behavior for breast cancer (Lechner et al., 1997b), and the other study investigated correlates of paying attention to cancer symptoms and helpseeking behavior (De Nooijer, 2001). Recently, the important role of past behavior as an independent predictor for future health behaviors such as exercise, has been discussed in relation to the influence of social cognitive variables on behavior (Norman and Conner, 1995).

Of the proximal variables, self-efficacy and social norms had a significant influence on adherence behavior. The influence of social norms was not found in other studies. In studies on the determinants of intention of health behavior in general, as well as studies on exercise behavior in particular, self-efficacy was found to be an important predictor as well (Godin and Kok, 1996; Kok et al., 1991; Lechner and De Vries, 1995; Marcus and Owen, 1992; McAuley, 1993). In our previous cross-sectional study with pretest data, self-efficacy abilities was also found a significant predictor of the intention to adhere to PFME therapy (see Chapter 3). In addition, self-efficacy has been found to predict adherence to disease management behaviors in women with heart disease, such as following dietary recommendations and exercising (Clark and Dodge, 1999), as well as adherence to medication taking (Brus et al., 1999). With regard to physiotherapeutic exercise therapy, self-efficacy was found to correlate significantly to exercise adherence (Sluijs et al., 1993).

Both cross-sectional and longitudinal analyses elucidated two significant predictors of long-term adherence to PFME therapy of the distal variables: UI symptoms and sex education at school. Women with more frequent weekly wet episodes before and one year after therapy, more likely retained a higher adherence level one year after therapy, 
than women with less frequent losses. This finding suggests that women adapted longterm adherence behavior to their symptoms. A similar adaptation pattern was found in the study by Burns et al. (1993), who argued that women with mild symptoms may not perceive their incontinence as a problem warranting sustained effort, while women with many daily losses recognize it as an ongoing problem and make a persistent effort as a positive response occurs. As stated, adherence level was high, but it is unclear whether women in this study had fewer wet episodes because their previous adherence (between pretest and posttest) was high or whether women had high adherence levels although they were dry or had mild UI symptoms. In contrast to others (Be and Talseth, 1996; Chen et al., 1999; Lagro-Janssen and Van Weel, 1998), we did not find that women with higher adherence levels had fewer wet episodes at follow-up. That having had sex education at school was a significant predictor of long-term adherence behavior is probably an artifact of the differences in this variable between conditions at pretest, although analyses did not reveal a significant interaction with condition.

The second aim of the study was to evaluate the impact of a specific theory-driven health education program supplemented to PFME therapy on adherence behavior and predictors of adherence. Adherence behavior of the women in this study was higher than found in other studies during a one year follow-up (Ferguson et al., 1990; Janssen et al., 2001; Lagro-Janssen et al., 1992). A significant effect of the program was only found in the longitudinal analysis at posttest: the group with the self-help guide had lower adherence levels than the group without the guide. But adherence behavior was not significantly different between conditions at posttest, and none of the other analyses revealed a significant contribution of the health education interventions or the self-help guide on adherence, nor on the predictors. An interesting explanation for the lack of impact of the health education program was found in the process evaluation. According to the remarks of the physiotherapists, working with the standardized protocol checklist for PFME therapy had structured the content of their therapy sessions compared to their treatment behavior in the past. Together with the fact that physiotherapists had to fill in a treatment form for every woman to evaluate treatment goals, it may be expected that evaluation moments and concurrent feedback during therapy have been structured as well. Our results thus suggest that having a standardized protocol checklist for PFME therapy covering all treatment aspects and evaluation moments, which is intensively guided by enthusiastic physiotherapists, seems most important for realizing long-term effects and optimal adherence behavior. 
In our study, every woman had such intensive oral guidance during PFME therapy. This might explain why the written health education program had no significant additional influence.

This study offered the advantage of presenting data related to theoretical constructs and results from longitudinal analyses, but it was also limited by several factors. A Hawthorne effect may have biased our findings (Cook and Campbell, 1979). All therapists in our study knew that they were in a trial evaluating the effectiveness of additional health education to regular PFME therapy. They may unintentionally have given more and structured attention to the regular patient education part of their therapies. Social desirability may also have played a role, but this was not assessed among physiotherapist. Among the women in this study, social desirability scores were high $(80 \%)$, which might explain the reporting of high adherence levels. Nevertheless, social desirability scores were equal between conditions and would therefore have influenced all scores in the same direction. Moreover, an attempt was made to minimize social desirability by using a five-point scale in stead of a dichotomous scale in the diary question on adherence behavior, in which the first three options referred to reasons for non-adherence, the fourth to moderate adherence and the fifth to optimal adherence. Another possibility is that the measurement of adherence itself could have functioned as a reminder for adherence behavior (Beurskens et al., 1992; Myers and Midence, 1998; Windsor et al., 1994), thereby significantly raising adherence levels in all conditions. If so, then it may be expected that the reminder phone calls for sending back the measurements would also have raised adherence levels (Burns et al., 1993; Nygaard et al., 1996). However, this was checked and no significant correlation was found between the number of calls per person and adherence behavior. Memory bias is not likely to have played a role in this study, because diaries are expected to prevent memory bias (Windsor et al., 1994).

\section{Implications for practice}

The amount of variance in long-term adherence to PFME therapy we were able to explain in this study, was high. Remarkable is that, based on theory and empirical data, many possible determinants of adherence had been identified in the needs assessment, while only a few appeared to be significant predictors of adherence behavior. Of these predictors, self-efficacy expectations and intention may be influenced through patient education. However, the self-help guide had no additional influence on these 
predictors, although it had included specific methods to enhance self-efficacy, such as setting realistic and attainable goals and providing feedback, as well as motivational messages to stimulate a positive intention to adhere. Furthermore, the PFME therapy itself had no influence on self-efficacy either and intention had significantly decreased over time. To compare, Kerssens and collegues (1999) developed and evaluated a training program for the enhancement of patient education skills in physiotherapy, aimed at better monitoring of adherence problems during treatment and enhancement of self-efficacy of patients regarding adherence behavior after treatment, but the program was not effective either (Kerssens et al., 1999). To conclude, our results suggest that is is not necessary to address so many cognitive proximal and distal variables in a health education program. Furthermore, the high adherence levels we found may be explained by the motivating effect of the enthusiasm of the physiotherapists as well as the intensively guided therapy sessions.

In addition, although we lack real proof whether the protocol checklist for PFME therapy (developed as a standard for all conditions) had optimized usual care, opinions of physiotherapists particularly confirmed that they worked more planned and systematically when using the protocol checklist. To reveal whether a structured therapy protocol such as used in our study really optimizes effectiveness of PFME therapy, a randomized controlled trial should be conducted to compare PFME therapy with checklist protocol to a control condition of PFME therapy without checklist protocol. The strongest argument in favor of considering implementation of the protocol checklist in existing practice guidelines for physiotherapeutic PFME therapy (see e.g. Berghmans et al., 1998a; Messelink et al., 2000), is that applying the protocol requires little investment, is easy to use and provides good insight into the activities of the physiotherapist. The use of the protocol can also be trained in the follow-up courses on pelvic floor dysfunctions. 


\title{
CHAPTER 5
}

\section{Effectiveness of pelvic floor muscle exercise therapy supplemented with a health education program to promote long-term adherence among women with urinary incontinence}

\begin{abstract}
A longitudinal randomized controlled trial was conducted to evaluate the effectiveness of physiotherapeutic pelvic floor muscle exercise (PFME) therapy supplemented with a health education program to promote long-term adherence among women with stress, mixed and urge urinary incontinence. Adherence was operationalized in terms of performing 100 slow and fast twitch contractions daily during activities, using sudden contractions to prevent leakage (the knack), training the bladder when needed, and integration of the use of the pelvic floor muscles in the daily posture and movement pattern. Outcome measures were weekly frequency of wet episodes and adherence behavior.

The health education program had no additional impact to individual contact during PFME therapy on treatment outcome, nor on adherence. However, the individual physiotherapeutic treatment protocol was very effective in reducing incontinence symptoms, and effects were maintained during the one-year follow-up period. Adherence was very high; most women followed the behavioral advice on average six days per week at posttest and still four to five days one year after therapy. Women who had relatively frequent wet episodes before and one year after therapy had higher adherence levels compared to women who had fewer weekly losses. This indicates that women learned to adapt adherence behavior to their symptoms. Results suggest that a standardized protocol checklist for physiotherapists covering all treatment aspects of PFME therapy, may optimize long-term treatment outcome and adherence behavior among patients, and outshines the addition of a health education program.
\end{abstract}

Manuscript submitted with some alterations as: Dianne Alewijnse, Job Metsemakers, Ilse Mesters, Bart van den Borne. Effectiveness of pelvic floor muscle exercise therapy supplemented with a health education program to promote long-term adherence among women with urinary incontinence 


\section{INTRODUCTION}

Urinary incontinence (UI) is defined by the International Continence Society (ICS) as the involuntary loss of urine which is objectively demonstrable and a social or hygienic problem (Abrams et al., 1988). The three most prevalent types are stress, urge and mixed UI. Stress incontinence is the complaint of involuntary loss of urine during, for instance, coughing, sneezing and physical exertion, such as sport activities and sudden changes of position. Urge incontinence is the complaint of involuntary loss of urine associated with a sudden, strong desire to void. In mixed UI, symptoms of both stress and urge incontinence are present (Abrams et al., 1988; Blaivas et al., 1997).

Today, PFME therapy has been proven effective for women with stress and mixed incontinence (Berghmans et al., 1998a; Bø, 1995a; Hay-Smith et al., 2001). Some studies reported favorable results for urge incontinence as well (Berghmans et al., 2000; Nygaard et al., 1996; Wyman et al., 1998). Therefore, PFME therapy is recommended as first-choice treatment for women with symptoms of stress, urge and mixed urinary incontinence when other physical impairments causing urinary incontinence have been excluded and possible urinary tract infections have been treated successfully. Overall, in studies up to $70 \%$ of the population was cured or improved after therapy (Berghmans et al., 1998a; Bø, 1995a; Burns et al., 1993; LagroJanssen et al., 1992; Nygaard et al., 1996), but success rates declined during follow-up as adherence to PFME therapy recommendations deteriorated. This finding can be illustrated by three studies that found that adherence, both during the period of therapy (including practicing at home) and thereafter, was a significant predictor of long-term success (Bø and Talseth, 1996; Chen et al., 1999; Lagro-Janssen and Van Weel, 1998). In one study, $62 \%$ of the women reported good adherence three months after therapy. This declined to $51 \%$ one year after therapy and to $39 \%$ at five-year follow-up, while in the meantime incontinence symptoms had returned or increased significantly (Lagro-Janssen et al., 1992; Lagro-Janssen and Van Weel, 1998). Chen et al. (1999) showed that during a two-year follow-up, continence success rates declined more in the groups with poor and moderate adherence despite symptoms than in the group that adhered well. Bø and Talseth (1996) found that those who exercised three times per week or more had significantly fewer symptoms than those who exercised less frequently, five years after organized PFME training. To conclude, an intervention aimed at promoting long-term adherence to PFME therapy seems warranted.

The promotion of adherence behavior is considered an integral part of patient education concerning physiotherapeutic PFME therapy. Various authors have pointed 
out that patient education in physiotherapy, and especially the part of encouraging adherence behavior, lacks a systematic and theoretically funded behavioral approach (Knibbe and Wams, 1994; Sluijs et al., 1993). A literature search was conducted to reveal the experience of others and their evaluation of interventions to improve adherence to PFME therapy so far. In four studies, the PFME program included adherence-promoting strategies: audiocassette tapes to guide exercising at home, telephone calls as reminders, and follow-up visits to provide feedback and reinforcement. One study found that after six weeks of training at home, adherence was significantly better in the group that had used an exercise tape, compared to the group without the tape (Gallo and Staskin, 1997). Surprisingly, in the other three studies adherence behavior itself was not measured (Burns et al., 1993; Nygaard et al., 1996) or not related to the adherence-promoting strategies (Wyman et al., 1998), thus the efficacy of these strategies remains unclear.

The observations concerning the link between adherence and therapy outcome, as well as the need for systematic and planned health education as an adherence-promoting strategy, led to the study described in this chapter. A longitudinal randomized controlled trial (RCT) on the effectiveness of PFME therapy supplemented with a theory-driven health education program was set up to promote long-term adherence to PFME therapy. It was hypothesized that better adherence behavior was related to better therapy outcomes, and the study addressed the following two research questions:

1. Does PFME therapy supplemented with a health education program result in better therapy outcomes than PFME therapy alone, one year after therapy?

2. Does PFME therapy supplemented with a health education program result in better adherence behavior than PFME therapy alone, one year after therapy?

\section{METHODS}

\section{Pelvic floor muscle exercise therapy}

The content of PFME therapy and the operationalization of adherence advice as applied in this study, were based on usual care in the Netherlands and recommendations of several authors (Berghmans et al., 1998b; Berghmans et al., 2000; Bø et al., 1990; Bø, 1995a; Lagro-Janssen et al., 1995; Miller et al., 1998; Mouritsen et al., 1991; Nygaard et al., 1996; Payne, 2000; Versprille-Fischer, 1995; Wyman et al., 1998). For the purpose of this study, a protocol for the individual PFME therapy was 
prepared in cooperation with the participating physiotherapists as a standard for all study conditions. This written protocol checklist covered all treatment aspects that are usually applied in PFME therapy during diagnostic assessment activities, formulation of the treatment plan, commonly given patient education, treatment of exercise therapy and evaluation (Knibbe and Wams, 1994; Verhulst et al., 1994; Versprille-Fischer, 1995). A schedule for frequency of treatment sessions over time, covering nine sessions of 30 minutes each in 14 weeks, or for additional sessions up to 22 weeks, was added to the protocol. We agreed that this protocol represented usual care in a written form.

PFME therapy for stress incontinence focused on the integration of the automatic and subconscious use of the pelvic floor muscles during the daily posture and movement pattern, and on the integration of pelvic floor muscle exercises (PFMEs) in daily life. For urge and mixed incontinence, the therapy focused first on bladder training in which a normal voiding frequency of approximately seven voidings per day is trained. Second, the therapy included the same program as for stress incontinence with the addition of learning to prevent leakage when feeling an urge, by contracting the pelvic floor muscles, which inhibits the contraction of the bladder. The therapy included teaching about the anatomy and function of the bladder, the pelvic floor muscles, the continence mechanism, toilet behavior and education on the importance of adherence to the advice.

Adherence to PFME therapy was operationalized as following four behavioral advice:

1) Perform PFMEs regularly as advised by the therapist: perform 10 slow twitch contractions (10-30 secs.) and 10 fast twitch contractions (2-3 secs.), both five times a day, each contraction being followed by relaxation. Increase repetitions in each session to the point of fatigue and incorporate different starting positions and functional activities into the exercise program. Check contractions with self-palpation.

2) Perform a correct toileting and drinking behavior: Sit straight on the toilet and relax the pelvic floor muscles during voiding, and drink 1500 to 2000 milliliters per day. Women with a deviant voiding frequency should train their bladder by timing their voidings during the day until a normal frequency of seven voidings a day is reached.

3) Using the 'knack'; prevent wet episodes by contracting the pelvic floor muscles when feeling a sudden urge to void (urge symptoms) or when coughing, sneezing or laughing (stress symptoms). 
4) Automatically and subconsciously use the pelvic floor muscles, especially during moments of abdominal pressure, in the daily posture and movement pattern to prevent leakage, thereby using a correct body posture and breathing technique.

These behaviors are expected to be performed during and after therapy, and after a relapse, women need to be able to restart and follow through with these behaviors. Optimal adherence during treatment is expected to be a prerequisite for long-term adherence. For example, it is unlikely that women who are not doing PFMEs on a regular daily basis during the period of therapy, will do so afterwards.

Furthermore, it should be noted that the objective of doing approximately 100 slow and fast twitch PFMEs per day, must be seen as a rough guideline. It is still unclear what intensity, frequency and duration of PFMEs are most effective. Individual differences may require a different exercise regimen and when the condition of the pelvic floor muscles has recovered through exercising, a lower exercise frequency or intensity may be equally effective in maintaining the condition of the pelvic floor (Berghmans et al., 1998b; Bø, 1995b; Wells, 1990).

\section{Development of the health education program}

On the basis of the Intervention Mapping (Bartholomew et al., 2001) approach, a series of specific steps and procedures for developing health education programs were conducted based on theory, empirical findings and real data (see Chapter 2). The specific health education program that was developed for this study, used theorydriven behavioral change methods based on social-cognition theory. This means that encouraging adherence to PFME therapy is viewed as a behavioral change process in which factors such as attitude towards adherence behavior, self-efficacy expectations, perceived severity of symptoms, and all kinds of barriers hampering adherence behavior are considered determinants of adherence to PFME therapy (De Vries et al., 1988; Leventhal and Cameron, 1987; Leventhal et al, 1998; Prochaska et al., 1992 and 1994).

For each determinant of adherence behavior identified in the needs assessment (see Chapter 2), specific aims were formulated and appropriate methods and strategies were selected. For example, for the method of goal setting, the strategy consisted of a filling-in task to write down personal treatment goals, and for the method of modeling, role model stories were the strategy (Bassett and Petrie, 1999; Strecher et al., 1995; see Chapter 2). Furthermore, quality principles for patient education were applied 
(Mullen et al., 1985). This implied among others, that information was targeted to women's needs, self-evaluation about changes in symptoms was stimulated, and reinforcement was given for positive behavior changes. In addition, we developed a minimal, a medium, and a maximum intervention that each followed the physiotherapeutic treatment plan in content, to enable investigating what intensity of health education would be necessary to promote adherence effectively. Three health education interventions were developed of which the most important components were reminders, guidance of adherence as self-management process, and structured feedback (Cramer, 1995; Leventhal and Cameron, 1987; Leventhal et al., 1998; Prochaska et al., 1992 and 1994):

1) Reminder intervention (Ri): since forgetting was most often mentioned as a barrier for adherence, this intervention consisted of a folder with information about PFME therapy and adherence behavior and several tips to remember adherence behavior. Stickers were included as reminders.

2) Reminder and Self-Help Guide intervention (R-SHGi): a guide (Alewijnse, 1997) addressing facts and myths about UI and pelvic floor muscles, coping with UI, tips to tackle all barriers hampering adherence behavior, and relapse prevention strategies to support the self-management process. The self-help guide also contained the stickers of the $\mathrm{Ri}$ and reminder tips.

3) Reminder, Self-Help Guide and Counseling intervention (R-SHG-Ci): identical to the R-SHGi, with the addition of a counseling scheme for physiotherapists, guiding structural oral feedback and reinforcement to promote adherence behavior.

\section{Participants}

The study was approved by both the Medical Ethical Committee of Maastricht University and the Maastricht University Hospital and the Review Committee of the Registration Network Family Practices (RegistratieNet Huisartspraktijken, RNH) (Metsemakers et al., 1992). Participants were recruited from the RNH, which consists of 23 general practices with 55 general practitioners (GPs), covering a population of around 100,000 persons, whose data on relevant chronic health problems and demographic variables are registered and continuously updated. Since many women do not seek medical treatment for UI (Shaw et al., 2001), community-dwelling women over 17 years old with at least one of the following risk factors for UI were selected: vaginal delivery, medical history of gynecological operations, asthma, arthritis, and 
obesity (Diokno et al., 1990; Milson et al., 1993; Snooks et al., 1990; Yarnell et al., 1982). Selected women received a short questionnaire through their GP. The questionnaire consisted of items on risk factors for UI, continence status, quality of life according to continence status and use of health care resources for UI. Women reporting symptoms of stress, urge or mixed UI were also asked if they were interested in individual PFME therapy and participation in a behavioral intervention study. This procedure was conducted twice; once in 1995 in a study on the prevalence of UI among 2914 women with risk factors for this condition (Weijts et al., unpublished data), and once in 1997 among 1341 women when the present behavioral intervention study was set up. In 1995, 320 women, when asked showed an interest in PFME therapy and 198 of them were still willing to do so in 1997. Of the second recruitment, 166 women were willing to participate, and a poster recruitment campaign in the participating general practices yielded another 12 women. Thus, a total of 376 women were interested in participation.

From January to October 1998, these 376 women received a letter from the researcher explaining that participation involved engaging in individual PFME therapy with a female physiotherapist and completing four questionnaires and diaries. The letter further instructed women to visit their GPs. After this letter, 180 women were actually willing to participate. The primary reasons given for refusal to participate by initially interested women were: symptoms of UI were minor or women were not sufficiently bothered by them to start PFME therapy (37\%), having other more severe health problems (14\%), having done PFME therapy or having been operated upon for UI $(12 \%)$, being too busy with work and/or care activities $(10 \%)$, no longer having symptoms of UI (5\%). Less frequently mentioned reasons were no interest in participation in a study, being afraid of vaginal palpation by the GP, feeling ashamed, being satisfied with the use of protective garments or medication, feeling too old to engage in PFME therapy, having no means of transport to the physiotherapist, or loss of mental capacity. Fifteen women had moved or died between the prevalence study and the start of the behavioral intervention study.

In total, 13 general practices with 36 GPs were involved in the selection of participants. Inclusion criteria were: ability to complete questionnaires, full understanding of the Dutch language and completion of the consent form. Excluded from the study were women (total $n=47$ ) without symptoms of stress, urge or mixed UI $(n=11)$ based on their anamneses (Lagro-Janssen et al., 1995), women suffering from neurological conditions such as MS, CVA and spina bifida or suffering from 
venereal disease $(n=5)$, women with viral infections $(n=4)$, women using medication for UI or using medication that enhances/influences UI $(n=1)$, women who were pregnant or within three months after delivery or women who had been operated upon for UI $(n=20)$, and women with physical impairments making PFME therapy impossible $(n=6)$. The GP also evaluated the presence of prolapse and vaginal atrophy, and the ability to voluntarily contract the pelvic floor muscles through vaginal palpation. Women with urinary tract infection were treated and included when cured.

\section{Physiotherapists}

We recruited 28 female physiotherapists specialized in the field of PFME therapy with an outpatient physiotherapy practice in the residential area of the participating general practices. Mean age of the therapists was 40.8 years (SD 8.9; range 24-63). They had been in practice for on average 17.5 years (SD 9.1; range 1-39), and had been specialized in PFME therapy for on average 6.4 years (SD 5.6; range 1-25). Mean working hours per week of these physiotherapists was 27.4 (SD 9.7; range 12-45). Yearly, they treat an average of 17 (SD 11; range 4-50) women with PFME therapy.

\section{Design and procedures}

A longitudinal RCT with three intervention and one control condition was conducted. The control condition consisted of individual physiotherapeutic PFME therapy alone, representing usual care. The intervention conditions consisted of PFME therapy supplemented with one of the three health education interventions. The two primary outcome measures were weekly frequency of wet episodes and number of days per week women had followed the behavioral advice of the physiotherapist, both measured with a 7-day diary (Kerssens et al., 1996; Nygaard and Holcomb, 2000; Wyman et al., 1988).

The four conditions were randomly allocated to the physiotherapists. However, due to a shortage of therapists and to minimize the chance of contamination, the control condition and $\mathrm{Ri}$ were allocated to the same physiotherapists. The 133 eligible women were randomized in one of the four conditions by the GP's assistants, who had been instructed by the researcher. A randomization form, containing codes for the four conditions and for the physiotherapists serving the residential area of a particular 
general practice, facilitated the procedure and ensured that every physiotherapist would treat about the same number of participants, and that conditions would be equally filled. In this way, both the GPs and their assistants, as well as the participants were blinded for intervention groups. Physiotherapists could not be blinded for the intervention. For practical reasons only, the researcher could not be blinded either.

The use of the standardized protocol checklist during PFME therapy was instructed to all physiotherapists by the researcher, and each therapist was asked to fill out a treatment form for every patient. These forms consisted of the participant's name and date of birth, the diagnosis of the GP on the referral letter and of herself, and a table to evaluate treatment goals. For each experimental condition, the standardized protocol checklist was supplemented with instructions on how and when to use the health education intervention during treatment. This was instructed separately to the group of physiotherapists of each condition by the researcher. The Ri and R-SHGi had to be given to the women at the onset of therapy. The instruction for the guide was, whenever a woman would like to do so, to read about what she had learned during the sessions at home, to use it as a diary by writing down the behavioral advice and/or to use it as a reminder during and after therapy. The R-SHG-Ci intervention was used in a similar way as the R-SHGi, with the difference that the instruction was more directive. Physiotherapists had to instruct women to read pages $\mathrm{x}$ to $\mathrm{y}$ of the guide that corresponded with the content of that particular therapy session. Furthermore, these physiotherapists had been instructed by the researcher to follow a counseling scheme with oral instructions corresponding to the design of the guide and to the iterative phases of the physiotherapeutic treatment plan (Verhulst et al., 1994). This counseling process was supposed to structurally guide patients from the awareness of the need for adherence, to taking up adherence behavior after relapses by providing oral feedback and reinforcement (Prochaska et al., 1992 and 1994).

\section{Questionnaire and diary}

Outcome variables for the effect evaluation were collected with self-administered questionnaires and 7-day diaries before (pretest), immediately after (posttest), and at 3 and 12 months after therapy. Process evaluation items were included in the posttest and in the 12-month follow-up. Women received the pretest survey from the GP's assistants before therapy and the posttest survey from the physiotherapist after the final PFME therapy session. The 3- and 12-month follow-up surveys were mailed to the 
participants. When women did not return the surveys within three weeks after sending, they were phoned and asked to complete the surveys and return them as soon as possible in the prepraid envelope. Reasons for the delay and other aspects related to PFME therapy discussed in the call, were recorded.

\section{UI symptoms}

The 7-day diary assessed frequency and amount (droplets, dashes, whole bladders) of wet episodes, and the number of protective garments used (nothing, panty shields, sanitary towels, incontinence pads) (Nygaard and Holcomb, 2000; Wyman et al., 1988). In the questionnaire, these three aspects were also assessed on the basis of the PRAFAB score (Vierhout, 1990), for validation of the diary assessment. The three items correlated significantly with the diary assessment $\left(\mathrm{L}_{s}=.551-.766, \mathrm{p}<.001\right)$. Another three items measured the duration of symptoms, the type of urinary incontinence and daily voiding frequency. Symptom distress was assessed with the Incontinence Quality of Life scale (IQOL, scale 22-110, $\alpha=.92$; Wagner et al., 1996), and symptom impact with the Incontinence Impact Questionnaire (IIQ-7, scale 0-15, $\alpha$ $=.79$; Uebersax et al., 1995). Higher IQOL scores indicate a better quality of life in relation to incontinence symptoms, whereas higher IIQ-7 scores indicate a larger impact of symptoms in daily life. Satisfaction and perceived improvement were assessed with five-point scales.

\section{Adherence behavior}

The 7-day diary assessed adherence in terms of number of days per week women had followed the behavioral advice of the physiotherapist at posttest and follow-ups (item A, Table 1). Three items (B-D, in Table 1) on adherence behavior in the questionnaire were used as validation for the diary assessment (Kerssens et al., 1996), that all correlated highly and significantly $\left(\mathrm{L}_{\mathrm{s}}=.388-.632, \mathrm{p}<.001\right)$ with the diary item. These three items assessed how often women had adhered to the behavioral advice the day and the week before, and a self-evaluation score for adherence on a scale from 1 to 10. Four other items in the questionnaire assessed with seven-point scales how difficult or easy women found the adherence advice to integrate the adherence behavior in daily life and the advice on toileting and drinking behavior.

\section{Background variables}

Social desirability was assessed using the Social Desirability scale (Visser and Breemhaar, 1989; $\alpha=.63$ ). Ten items such as 'If I make a mistake, I will always be 
Table 1. Instruments for assessment of adherence behavior, and cross-sectional results at posttest, 3 - and 12 months follow-up ( 12,13 , $t 4) ;$ number $(\%)$ or $M(S D)$.

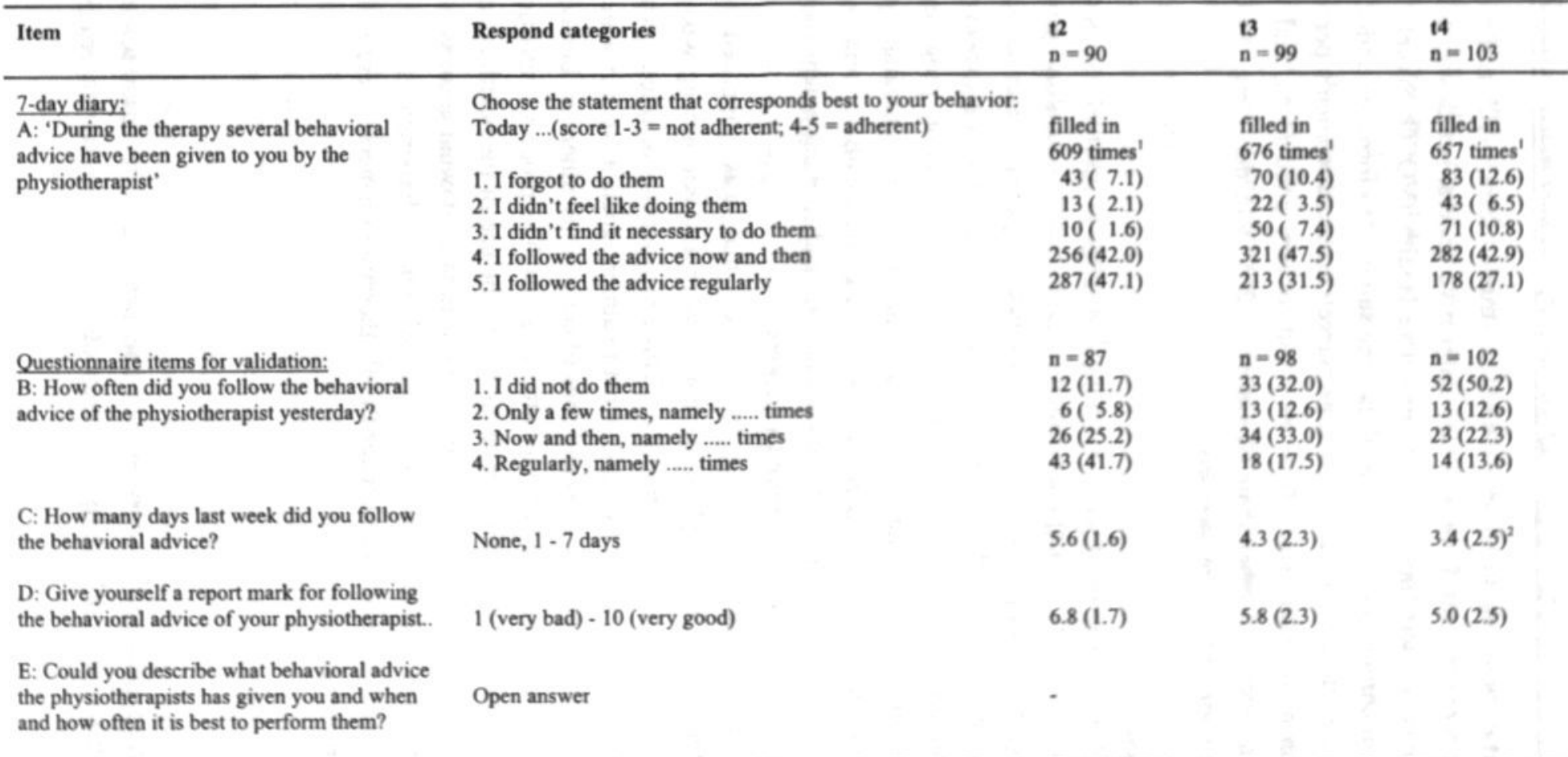

1. Most women answered this question every day in the 7-day diary, thus the maximal number of answers is the number of women completing that diary item $x$.

2. Kruskal Wallis test revealed difference among conditions; control $>\mathrm{R}-\mathrm{SHGi}, \mathrm{p}=.024$; rest of comparisons are not significant. 
prepared to admit it', were measured on a VAS scale (true -- false, 0-10), and summed to form an index score ranging from 0 to 100 . Subjective general health was assessed with the abbreviated 13-item 'Inventory of Subjective Health' (VOEG; Sikkel, 1980). The 13 items about chronic physical complaints were summed to form one index score (0-13). Two items of the Dutch National Health Survey assessed women's perception of their health on a five-point scale from very bad to very good (1-5), and which chronic conditions they had other than UI (index, 0-26) (Van den Berg, 1992). In addition, sociodemographics were assessed.

\section{Process evaluation}

Both participants and physiotherapists completed a process evaluation assessing satisfaction with and level of implementation of the health education program. Twenty-two structured and open items assessed women's experience and satisfaction with the therapy, and whether the therapy had lived up to women's expectations. Women's opinion about and satisfaction with the health education program was assessed with another 22 structured and open questions. For women in the experimental conditions, level of implementation was assessed with 15 items asking whether women had read the health education materials and to what extent they had used the filling-in tasks, the tips and reminder stickers.

Implementation of the oral counseling intervention that was supposed to be implemented in the therapy by the physiotherapists, were assessed among women in the R-SHG-Ci and all physiotherapists. Thirteen items assessed whether physiotherapists had discussed, for example, what treatment goals a woman wanted to reach in order to set small subgoals, pros and cons of continuous adherence behavior or possibilities of taking up adherence behavior after relapses. The process evaluation for physiotherapists further assessed their satisfaction with the use of the health education interventions during the PFME therapy and their estimate of what aspects of the interventions are most effective in promoting adherence. Experiences with the implementation of the protocol checklist for PFME therapy in general, were assessed among all physiotherapists.

\section{Analyses}

Data were analyzed using SPSS version 9.0, and procedures for data screening, randomization check and selectivity of dropouts, were applied according to 
Tabachnick and Fidell (1996). Descriptive statistics were used to describe sociodemographic variables and process evaluation findings. Univariate one-way analysis of variance and Kruskal Wallis tests were used to compare outcome variables at posttest, 3- and 12-month follow-up between conditions. Univariate analysis of covariance controlling for pretest values was used to compare the efficacy of the experimental conditions to the control condition on outcome variables at 12-month follow-up. When homogeneity of variances was lacking, or when group sizes were unequal and small, Kruskal Wallis tests with the change scores were applied.

No significant interactions of condition and weekly frequency of wet episodes with possible confounders were found through linear multiple regression analyses. Repeated measurement analyses of variance were used to detect changes over time with post hoc paired samples t-test with Holm's bonferroni correction ( $\alpha=.05 / \mathrm{n}$ tests) to reveal differences between measurements. Subgroup analyses for diagnostic groups were performed using the diagnosis of the physiotherapist as that determined therapy content.

An a priori power analysis (Bouter and Van Dongen, 1995) indicated that a sample size of 192 (48 per group) was needed to provide at least $90 \%$ power to detect a minimum difference between experimental conditions and control condition of $25 \%$ in weekly wet episodes $(p=.05)$ at 12 -month follow-up, when women in the control condition would improve by $50 \%$ (Lagro-Janssen, et al., 1992; Mouritsen et al., 1994). Although a size of 192 participants was not reached, analysis with $\mathrm{n}=103$ would still provide a power of $79 \%$ when predicted data from initial power analyses were found.

\section{RESULTS}

\section{Non-response analysis}

Four women declined to complete the pretest questionnaire and never started PFME therapy. Three of them had medical problems (symphiolysis, rheumatic complaints, severe tooth pain), were unable to do PFME therapy and should have been excluded before by the GP. One women claimed that she wanted to complete the questionnaire and start therapy, but never did, regardless of reminder calls.

Loss to follow-up numbers were not significantly different between intervention groups; control 3/32, Ri 7/29, R-SHGi 9/34, R-SHG-Ci $7 / 34$. Twenty-six women $(20 \%$ of 129) withdrew either immediately after randomization $(n=10)$, during the treatment 
period $(n=4)$, or during the follow-up measurements $(n=12)$. Reasons or combinations of reasons for withdrawal included personal illness $(n=12)$ and/or an ill relative $(n=3)$, stressful life or busy schedule $(n=8)$, transportation problems $(n=2)$, symptoms not improved $(n=2)$, lack of interest $(n=7)$. One women died after the 3 month follow-up and one woman found out that she went to the toilet only three times a day through completing the pretest diary. Knowing this, she started to toilet more often and by doing so, eliminated the UI symptoms and no longer needed PFME therapy.

The 26 women lost to follow-up differed from the women who completed the study on two variables at pretest. The women lost to follow-up had more frequent $(\underline{U}=978.5, \underline{p}$ $=0.029)$ and more severe $(\underline{U}=1036.5, \underline{\mathrm{D}}=0.045)$ wet episodes as measured with the questionnaire. All analyses, except intention to treat (last observation carried forward), were performed with the 103 women who completed the 12-month follow up measurements. Of those 103 women, the posttest of 15 women and the 3-month follow-up of two women were not returned due to unknown reasons.

\section{Characteristics of participants}

Sociodemographic characteristics as well as outcome variables of incontinence symptoms at pretest of the 129 remaining participants are presented in Table 2 . The youngest woman was 27 , the oldest 82 . Most women had a partner and children, and one in three women still had children living at home. Of the 45 women with a paid job, $40 \%$ worked more than three days per week. More women had a low education level as compared with the general Dutch population (www.cbs.nl, 2001), and two-third of the women had sex education at school. Most women were insured under the Social Health Insurance scheme.

Mean body mass index was 26.9 (SD 4.8), which is slightly above normal. Twice as many physical complaints (VOEG) were reported as found among middle-aged

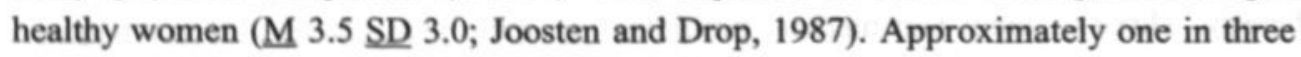
women evaluated their own health status as good, one in four as not so good, two in five as moderate, and four in five women mentioned having other chronic diseases. Experiences of sexual violence in the youth and in adult life were reported by 22 $(17.1 \%)$ and $16(12.4 \%)$ women, respectively.

Physiotherapists diagnosed $37.2 \%$ of the women with stress, $31.0 \%$ with mixed and $8.5 \%$ of the women with urge incontinence, but unfortunately missed to fill-out the 
Table 2. Characteristics of participants, $\mathrm{N}=129$

Characteristics of study population Number of

Percentage of

Mean (SD)

women

population

Age

Having a partner

107

Having children

118

$82.9 \%$

$91.5 \%$

Number of children

Having a paid job

45

$55.6(10.9)$

Hours of paid labor per week

Education Level $(n=127)$

$$
\begin{aligned}
& \text { Low' } \\
& \text { Medium } \\
& \text { High }
\end{aligned}
$$

Had sex education at school

Social Health Insurance

Recruitment phase 1995

Recruitment phase 1998

Body mass index

VOEG

General health status

Good
Moderate
Poor

Poor

Other chronic conditions (0-26)

Sexual violence in youth

Sexual violence in adult life
96

16

15

43

98

69

60

$\begin{array}{ll}46 & 35.7 \% \\ 52 & 40.3 \% \\ 31 & 24.0 \% \\ & \\ 22 & 17.1 \% \\ 16 & 12.4 \%\end{array}$

Diagnosis of physiotherapist $(\mathrm{n}=99)$

$\begin{array}{lrr}\text { Stress } & 48 & 37.2 \% \\ \text { Urge } & 11 & 8.5 \% \\ \text { Mixed } & 40 & 31.0 \% \\ \text { missing } & 30 & 23.3 \%\end{array}$

Duration of symptoms $(n=127)$

$\begin{array}{llr}\text { less than } 2 \text { years } & 11 & 8.7 \% \\ 2-7 \text { years } & 72 & 56.7 \% \\ \text { over } 7 \text { years } & 44 & 34.6 \%\end{array}$

Frequency of wet episodes

Max. 1x per week

2-7x per week

Several times a day

Amount of urinary loss

Droplets

Droplets and dashes

Dashes

Dashes and whole bladders

Whole bladders

$8 \quad 6.2 \%$

Weekly frequency of wet episodes, 
dagnosis of the other women $(n=30)$. Most women reported having symptoms of moderate severity for several years and the majority of women lost dropplets and dashes on several days per week. Mean weekly frequency of wet episodes was 24.5 (SD 25.0). Women occasionally worried about their incontinence symptoms (IQOL) and wet episodes had some negative impact on daily life activities (IIQ-7).

Significant differences between conditions at pretest were as follows: women in the control condition used heavier pads than women in the R-SHGi $(\underline{H}(3)=9.143, \underline{p}=$ $.027)$, and women in the Ri experienced more symptom distress than women in the RSHG-Ci $(\mathrm{F}(3,124)=3.079, \mathrm{p}=.030)$ and more symptom impact than women in the $\mathrm{R}$ SHGi $(\mathrm{E}(3,124)=3.105, \mathrm{R}=.029)$. Further, fewer women in the control condition had sex education at school than women in the R-SHGi $(\underline{H}(3)=4.098, p=.043)$. With $n=$ 129 , no differences were found among groups on any of the other variables at pretest. However, with $n=103$, pretest scores of weekly frequency of wet episodes were significantly higher $(\underline{\mathrm{H}}(3)=8.943, \mathrm{p}=.030)$ in the control $(\underline{\mathrm{M}} 27.6, \underline{\mathrm{SD}} 21.9)$

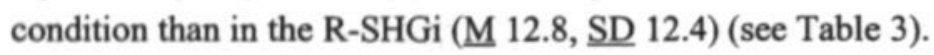

\section{Implementation of health education interventions}

The assessment of the level of implementation of the three interventions among women and physiotherapists revealed that both the reminder and the counseling intervention had not been implemented as planned. While the therapists thought that most women would use the stickers, two in five women of the Ri and the R-SHG-Ci actually used a few stickers, and only two women in the R-SHGi did so. Thus, the use of stickers and other reminders suited only some of the women, regardless of the condition to which they had been allocated. Furthermore, no relation was found between the use of stickers and adherence behavior.

The assessment of the level of implementation among physiotherapists after the intervention period had ended, revealed that all 13 key aspects of oral counseling had been equally applied by the physiotherapists in all conditions, although only the therapists in the R-SHG-Ci had actually been trained in applying the specific counseling scheme to provide structural oral feedback and reinforcement.

Thus, having had a self-help guide remained as the only distinction between the study groups. Therefore secondary analyses were conducted in which women in the two selfhelp guide groups ( $\mathrm{R}-\mathrm{SHGi}+\mathrm{R}-\mathrm{SHG}-\mathrm{Ci}$ ) were compared with women in the other two 
Table 3. Mean (SD) of weekly frequency of wet episodes (7-day diary) and adherence behavior as number of days per week women followed the behavioral advice, for each condition and total group. Differences between conditions and changes between pretest ( $t 1)$, posttest ( $t 2), 3$ - and 12 -month follow-up ( $\mathrm{B}$ and $\mathrm{t} 4)$.

\begin{tabular}{|c|c|c|c|c|c|c|}
\hline Variable & $\begin{array}{l}\text { Control } \\
\mathrm{n}=29\end{array}$ & $\begin{array}{l}\mathbf{R i} \\
\mathrm{n}=\mathbf{2 2}\end{array}$ & $\begin{array}{l}\text { R-SHGi } \\
\mathbf{n}=\mathbf{2 5}\end{array}$ & $\begin{array}{l}\text { R-SHG-Ci } \\
\mathrm{n}=27\end{array}$ & $\begin{array}{l}\text { Total } \\
\mathrm{n}=103\end{array}$ & $\begin{array}{l}\text { between } \\
\text { groups } \\
\mathbf{p}^{1}\end{array}$ \\
\hline \multicolumn{7}{|l|}{$\begin{array}{l}\text { wet } \\
\text { episodes }\end{array}$} \\
\hline $\mathrm{tl}$ & $\begin{array}{l}\mathrm{n}=29 \\
27.6(21.9)\end{array}$ & $\begin{array}{l}\mathrm{n}=21 \\
31.2(31.1)\end{array}$ & $\begin{array}{l}\mathrm{n}=25 \\
12.8(12.4)\end{array}$ & $\begin{array}{l}n=27 \\
20.7(25.8)\end{array}$ & $\begin{array}{l}\mathrm{n}=102 \\
22.9(24.1)\end{array}$ & $\begin{array}{l}.030 \\
\mathrm{C}>\mathrm{SHGi}\end{array}$ \\
\hline 12 & $\begin{array}{l}\mathrm{n}=24 \\
7.6(11.5)\end{array}$ & $\begin{array}{l}\mathrm{n}=18 \\
10.0(13.2)\end{array}$ & $\begin{array}{l}\mathrm{n}=22 \\
5.2(9.2)\end{array}$ & $\begin{array}{l}\mathrm{n}=23 \\
6.7(18.9)\end{array}$ & $\begin{array}{l}\mathrm{n}=87 \\
7.3(13.6)\end{array}$ & ns \\
\hline t3 & $\begin{array}{l}\mathrm{n}=29 \\
11.1(14.9)\end{array}$ & $\begin{array}{l}\mathbf{n}=20 \\
13.1(18.5)\end{array}$ & $\begin{array}{l}\mathrm{n}=25 \\
5.2(8.7)\end{array}$ & $\begin{array}{l}n=25 \\
6.6(13.6)\end{array}$ & $\begin{array}{l}\mathrm{n}=99 \\
8.8(14.3)\end{array}$ & ns \\
\hline 14 & $\begin{array}{l}\mathrm{n}=29 \\
8.8(13.0)\end{array}$ & $\begin{array}{l}n=22 \\
12.2(16.6)\end{array}$ & $\begin{array}{l}n=25 \\
4.2(5.5)\end{array}$ & $\begin{array}{l}\mathrm{n}=26 \\
6.5(10.8)\end{array}$ & $\begin{array}{l}\mathrm{n}=102 \\
7.8(12.2)\end{array}$ & ns \\
\hline in time, $\mathrm{p}^{2}$ & $\begin{array}{l}\mathrm{E}=10.021, \\
\mathrm{D}<.001 ; \\
\mathrm{t} 1>\mathrm{t} 2, \mathrm{t} 3, \mathrm{t} 4 \\
<.001 \\
\mathrm{t} 3>\mathrm{t} 4, .013\end{array}$ & $\begin{array}{l}\mathrm{E}=3.664 \\
\mathrm{z}=.039 \\
\mathrm{t} 1>2, .009 \\
\mathrm{t} 1>t 3, .002 \\
\mathrm{t} 1>t 4, .003\end{array}$ & $\begin{array}{l}\mathrm{E}=6.805 \\
\mathrm{z}=.003 \\
\mathrm{t} 1>\mathrm{t} 2, \mathrm{t} 3, \mathrm{t} 4 \\
<.001\end{array}$ & $\begin{array}{l}\mathrm{E}=4.912 \\
\mathrm{z}=.010 \\
\mathrm{t} 1>\mathrm{t} 2, \mathrm{t} 3 \\
.002 \\
\mathrm{t} 1>\mathrm{t} 4, .001\end{array}$ & $\begin{array}{l}\mathrm{E}=23.186, \\
\mathrm{Q}<.001 \\
\mathrm{t} 1>\mathbf{2}, \mathrm{t} 3, \mathrm{t4} \\
<, 001\end{array}$ & \\
\hline \multicolumn{7}{|l|}{ adherence } \\
\hline 12 & $\begin{array}{l}\mathbf{n}=24 \\
6.3(1.1)\end{array}$ & $\begin{array}{l}\mathrm{n}=18 \\
6.5(1.2)\end{array}$ & $\begin{array}{l}\mathrm{n}=22 \\
6.2(1.2)\end{array}$ & $\begin{array}{l}\mathrm{n}=23 \\
6.0(1.4)\end{array}$ & $\begin{array}{l}\mathrm{n}=87 \\
6.2(1.2)\end{array}$ & ns \\
\hline $\mathrm{t} 3$ & $\begin{array}{l}\mathrm{n}=28 \\
5.2(2.0)\end{array}$ & $\begin{array}{l}\mathrm{n}=20 \\
5.5(2.1)\end{array}$ & $\begin{array}{l}\mathrm{n}=23 \\
6.1(1.6)\end{array}$ & $\begin{array}{l}\mathrm{n}=25 \\
5.4(2.1)\end{array}$ & $\begin{array}{l}\mathrm{n}=96 \\
5.5(2.0)\end{array}$ & ns \\
\hline $\mathrm{t} 4$ & $\begin{array}{l}\mathrm{n}=28 \\
5.3(2.3)\end{array}$ & $\begin{array}{l}\mathrm{n}=20 \\
5.3(2.3)\end{array}$ & $\begin{array}{l}\mathrm{n}=24 \\
4.4(2.5)\end{array}$ & $\begin{array}{l}n=25 \\
4.4(2.3)\end{array}$ & $\begin{array}{l}\mathrm{n}=97 \\
4.8(2.4)\end{array}$ & ns \\
\hline in time, $\mathrm{p}^{2}$ & ns & ns & $\begin{array}{l}\mathrm{E}=5.651, \\
\mathrm{E}=.013 ; \\
\mathrm{t}>\mathrm{t} 4, .006 \\
\mathrm{t} 3>\mathrm{t} 4, .001\end{array}$ & $\begin{array}{l}\mathrm{E}=5.694, \\
\mathrm{D}=.011 ; \\
\mathrm{t}>\mathrm{t} 4, .002\end{array}$ & $\begin{array}{l}\mathrm{E}=14.323, \\
\mathrm{p}<.001 ; \\
\mathrm{t}>\mathrm{t} 3, .002 \\
\mathrm{t}>\mathrm{t} 4, \\
<.001 \\
\mathrm{t} 3>\mathrm{t} 4, .001\end{array}$ & \\
\hline
\end{tabular}

1. One-way ANOVA, comparison between interventiongroups per measurement with post hoc Dunnet's $\mathrm{C}$ test, or Kruskal Wallis.

2. One-way Repeated Measurement ANOVA with post hoc paired t-test with Holm's bonferroni correction for multiple comparisons. 
groups (Control condition $+\mathrm{Ri}$ ), to study the possible surplus value of the self-help guide on adherence behavior and determinants. To check for consistency of the results, women in the self-help guide groups were also compared with women in the control condition alone.

\section{Effect on incontinence symptoms}

The effectiveness of PFME therapy on incontinence symptoms was compared between the four intervention conditions, as well as between the group with and the group without the self-help guide. After adjustment for pretest scores, no significant differences were found between conditions in weekly frequency of wet episodes at posttest, 3- and 12-month follow-up. Mean weekly frequency of wet episodes was drastically reduced from an average of 23 losses per week to an average of eight losses per week, and remained fairly stable up to one year after therapy (Table 3). Further, relative cure and improvement rates of weekly frequency of wet episodes are presented in Table 4. Overall, $74.8 \%$ (intention to treat $64.4 \%$ ) of the women were cured or improved by $50 \%$ or more one year after therapy.

\begin{tabular}{|c|c|c|}
\hline Change & $\begin{array}{l}\text { Number of women } \\
(\%), n=103\end{array}$ & $\begin{array}{l}\text { intention to treat }(\%) \text {, } \\
\mathrm{n}=129\end{array}$ \\
\hline $100 \%$ (dry) & $38(36.9 \%)$ & $41(31.8 \%)$ \\
\hline $75-99 \%$ & $17(16.5 \%)$ & $20(15.5 \%)$ \\
\hline $50-74 \%$ & $22(21.4 \%)$ & $22(17.1 \%)$ \\
\hline $1-49 \%$ & $16(15.5 \%)$ & $19(14.7 \%)$ \\
\hline $0 \%$ or deteriorated & $8(7.8 \%)$ & $22(17.0 \%)$ \\
\hline missing & $2(1.9 \%)$ & $5(3.9 \%)$ \\
\hline
\end{tabular}

Subgroup analyses per diagnostic group were conducted and no differences were found between conditions at posttest, 3- and 12-month follow-up after adjustment for pretest scores. Further, no differences were found in weekly frequency of wet episodes at pretest between women with stress $(\mathrm{M} 21.2 \underline{\mathrm{SD}} 25.1, \mathrm{n}=41)$, mixed $(\underline{M} 22.5 \underline{\mathrm{SD}} 24.0$, $\underline{\mathrm{n}}=34)$ and urge UI (M $27.6 \underline{\mathrm{SD}} 20.7, \underline{\mathrm{n}}=9$ ), nor at posttest, 3- and 12-month followup after adjustment for pretest scores. Analyses based on women's self-diagnosis 
revealed the same results. Thus, PFME therapy yielded similar results for women with either stress-, urge- or mixed incontinence.

Other possible confounders for UI symptoms did not influence our results either. Using regression analyses, no significant interactions were found of both intervention condition and weekly frequency of wet episodes and possible confounders. The number of treatment sessions attended was not related to weekly frequency of wet episodes at pretest and had no influence on treatment outcomes. This indicates that all women had enough treatment sessions to benefit optimally. Mean number of treatment sessions was 8.5 (SD 2.9), although most women found fewer sessions enough ( $\mathrm{M} 6.6$ $\underline{\mathrm{SD}} 2.8 ; \mathrm{t}(88)=7.402, \mathrm{p}<.001)$. Only those women who did not have enough control over their pelvic floor muscles during daily activities and those who could progress further, had more than nine sessions.

\section{Adherence behavior}

Overall, adherence behavior was very high with women following the behavioral advice on average six days per week at posttest and four to five days per week one year after therapy (Table 3). A significant decrease in adherence behavior over time was found for the two groups with the self-help guide, and not in the two groups without the guide. Then, analyses were performed to check for differences in adherence behavior between study conditions, after adjustment for pretest frequency of wet episodes per week. No significant differences were found between the four study conditions in the number of days per week women had followed the behavioral advice at posttest, 3- and 12-month follow-up. The secondary analyses did not change this finding. Short- and long-term adherence behavior, or the change in adherence between posttest and follow-ups was not different for the group with the self-help guide compared to the group without the self-help guide. Overall, $67 \%$ of the women followed the behavioral advice four to seven days per week one year after therapy.

At posttest, women with stress or mixed UI had followed the behavioral advice more days per week (six) than women with urge UI (five) $(\mathrm{F}(2,72)=3.690, \mathfrak{R}=.030$ ), whereas no differences were found between diagnostic groups in adherence behavior at 3- and 12-month follow-up. Adherence behavior for each diagnostic group was also compared between intervention conditions, again after adjustment for pretest frequency of wet episodes. Adherence behavior at posttest, 3- and 12-month follow-up, did not differ between conditions for women with either, stress, urge or mixed UI. 
Table 1 presents details on adherence behavior for the total group, as the health education program had no significant additional effect on adherence. The summed diary data (A) revealed that most women had exercised now and then or regularly, and the latter group decreased in size over time. This finding is also reflected in women's self-evaluation of their adherence behavior on a scale from 1 to 10 , which declined from seven immediately after therapy to five one year later. On the questionnaire item 'How often did you follow the behavioral advice of the physiotherapist yesterday' (B), most women reported 'now and then' or 'regularly.' However, the actual frequency was often not filled in and there was a great variation between the women who had completed those open questions; they had exercised between 1 and 100 times per day at posttest $(n=68), 1-40$ times at 3-months follow-up $(n=61)$, and 1-20 times at 12months follow-up $(n=43)$. Because of the wide range and indistinct open answers $(E)$, it was not clear what women meant (e.g. sessions or actual repetitions; PFMEs or bladder training), and whether they had, in the case of PFMEs, performed 50 slow and 50 fast twist contractions per day in five sessions.

One year after therapy, women were also asked whether they found following the behavioral advice difficult or not. The integration of the behavioral advice in daily life activities was found neither difficult, nor easy, and most women expected to be able to continue their adherence behavior in the future under all circumstances, now and then $(30 \%)$ or regularly $(50 \%)$ at 12 months follow-up. Further, more women found performing the recommended exercises every day difficult (37.9\%) than easy $(26.2 \%)$. One year after therapy, most women (64.1\%) found drinking $1.5-2$ liters a day rather difficult, while the others found that easy. Over two-third of the women (65.1\%) found not going to the toilet every time when feeling an urge a little difficult before therapy, which had improved one year after therapy. This was, as might be expected, more difficult for women with urge and mixed UI than for women with stress UI. Overall, voiding frequency was normal and significantly decreased from 8 (range 2-18) to 6 or 7 (range 3-15) voidings per day $(\mathrm{F}(3,71)=15.202, \mathrm{p}<.001)$ between pretest and posttest, and remained stable afterwards. Women with mixed UI voided more often per day (M 9.4 $\underline{\mathrm{SD}} 2.8)$ before therapy than women with stress UI (M $7.3 \underline{\mathrm{SD}} 2.9)$ (F $(2,67)$ $=4.376, p=.016$ ), but after therapy no differences in voiding frequency were found between diagnostic groups. More than half of the women (55.3\%) found that their toilet behavior had improved a little, while the others had remained the same or their toilet behavior was already as recommended at the onset of therapy. 


\section{Relation between outcome variables}

When assessed with the total group scores, higher pretest weekly frequency of wet episodes were significantly related to higher adherence levels one year after therapy $\left(r_{4}\right.$ $=.385, \mathrm{p}<.001$ ). To further analyse the relation between frequency of wet episodes and adherence behavior, the total group of women was split up into four subgroups on the basis of pretest scores of weekly frequency of wet episodes and compared with changes in adherence behavior. Because of pretest differences in the weekly frequency of wet episodes between conditions, we also checked distribution of conditions among the four quartiles, with the following results (Table 5). Regardless of the treatment condition, women with the lowest weekly frequency of wet episodes at pretest and also at 12-month follow-up, had reduced adherence levels significantly between posttest and 12-month follow-up, while women with a higher weekly frequency of wet episodes at pretest and at 12-month follow-up, retained their high adherence levels at all measurements. These findings should be interpreted with caution because the numbers per group were very small. Thus, the fact that women in the two groups with the self-help guide had significantly lowered their adherence levels over time (see Table 3$)$, is a consequence of the fact that more women $(n=31)$ from the self-help guide conditions were present in the lowest two quartiles of the weekly wet episodes than in the highest two quartiles $(\mathrm{n}=18)$, as compared to 19 women from the control condition or $\mathrm{Ri}$ in the lowest two quartiles and 29 in the highest two quartiles. This indicates that most women learned to adapt their adherence behavior to their symptoms, and not only those who used the self-help guide. Thus, women with more frequent weekly wet episodes more likely remained a higher adherence level one year after therapy, than women with less frequent losses.

Weekly frequency of wet episodes was significantly related to symptom distress (IQOL) and symptom impact (IIQ-7). Women who had more frequent wet episodes had more symptoms distress $\left(r_{s}=-.389, p<.001\right)$, and experienced a greater impact of symptoms on daily life activities $\left(r_{s}=.316, p<.001\right)$. The quality of life scales IQOL and IIQ-7 were highly correlated $\left(\mathrm{r}_{\mathrm{s}}=-.594, \mathrm{p}<.001\right)$; women who had more worries about their symptoms, also experienced a greater impact of symptoms on daily life activities. Overall, adherence behavior was not related to these condition-specific quality of life scales. Only, women who had experienced more symptom distress at pretest, had higher adherence levels at posttest $\left(\mathrm{L}_{\mathrm{s}}=-.267, \mathrm{p}=.012\right)$, and one year after therapy $\left(\mathrm{r}_{\mathrm{s}}=-.270, \mathrm{p}=.007\right)$. 
Table 5. Adherence at posttest, 3- and 12-month follow-up $(t 2, t 3, t 4)$ per quartile subgroup of pretest weekly frequency of wet episodes and per treatment condition.

\begin{tabular}{|c|c|c|c|c|c|}
\hline $\begin{array}{l}\text { Quartile mean } \\
\text { (SD) frequency } \\
\text { from pretest to } \\
\text { t4 }\end{array}$ & $\begin{array}{l}\text { Treatment } \\
\text { condition }\end{array}$ & $\begin{array}{l}\text { Adherence at } \\
\text { t2, } \\
\text { no. women } \\
\text { mean (SD) }\end{array}$ & $\begin{array}{l}\text { Adherence at } \\
\mathrm{t} 3 \text {, } \\
\text { no. women } \\
\text { mean (SD) }\end{array}$ & $\begin{array}{l}\text { Adherence at } \\
\text { t4, } \\
\text { no. women } \\
\text { mean (SD) }\end{array}$ & $\begin{array}{l}\text { differences in } \\
\text { adherence in } \\
\text { time per quartile } \\
\text { and condition' }\end{array}$ \\
\hline $1: \begin{array}{c}2.0 \rightarrow \\
(1.4)\end{array} \quad \begin{array}{c}1.0 \\
(1.6)\end{array}$ & $\begin{array}{l}\text { Control } \\
\text { Ri } \\
\text { R-SHGi } \\
\text { R-SHG-Ci }\end{array}$ & $\begin{array}{l}3: 6.0(1.0) \\
3: 7.0(0.0) \\
8: 6.1(1.4) \\
9: 5.1(1.7)\end{array}$ & $\begin{array}{l}4: 4.3(1.0) \\
4: 5.8(1.9) \\
8: 5.8(2.5) \\
9: 4.3(2.4)\end{array}$ & $\begin{array}{l}4: 5.0(2.4) \\
4: 4.7(2.1) \\
8: 3.5(2.7) \\
9: 3.1(2.3)\end{array}$ & $\begin{array}{ll}\text { ns } & \\
\text { ns } & .050^{2} \\
\text { ns } & \\
.024 & .002^{2}\end{array}$ \\
\hline 2: $\begin{array}{c}9.7 \rightarrow 3.0 \\
(2.7)\end{array}$ & $\begin{array}{l}\text { Control } \\
\text { Ri } \\
\text { R-SHGi } \\
\text { R-SHG-Ci }\end{array}$ & $\begin{array}{l}6: 6.3(1.0) \\
5: 6.8(0.4) \\
7: 6.6(0.8) \\
6: 6.8(0.4)\end{array}$ & $\begin{array}{l}6: 5.8(1.3) \\
5: 6.0(1.0) \\
8: 6.1(1.1) \\
6: 6.3(0.5)\end{array}$ & $\begin{array}{l}7: 4.1(2.3) \\
4: 6.0(1.2) \\
8: 4.3(2.3) \\
6: 4.8(1.5)\end{array}$ & $\begin{array}{ll}.024 & \\
\text { ns } & .013^{2} \\
.034 & \\
.014 & .001^{2} \\
\end{array}$ \\
\hline 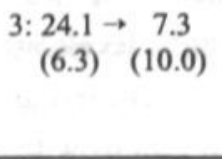 & $\begin{array}{l}\text { Control } \\
\text { Ri } \\
\text { R-SHGi } \\
\text { R-SHG-Ci }\end{array}$ & $\begin{array}{l}9: 6.6(0.9) \\
6: 6.0(2.0) \\
6: 6.0(1.5) \\
4: 6.0(0.8)\end{array}$ & $\begin{array}{l}9: 5.8(1.6) \\
6: 5.7(2.8) \\
5.6 .4(0.5) \\
5: 5.2(3.0)\end{array}$ & $\begin{array}{l}9: 5.1(2.8) \\
6: 5.2(2.8) \\
6: 5.2(2.8) \\
6: 4.5(2.9)\end{array}$ & $\begin{array}{ll}\mathrm{ns} & \\
\mathrm{ns} & \mathrm{ns}^{2} \\
\mathrm{~ns} & \\
\mathrm{~ns} & \mathrm{~ns}^{2} \\
\end{array}$ \\
\hline $\begin{array}{c}4: 60.0 \rightarrow 22.7 \\
(20.9)(15.8)\end{array}$ & $\begin{array}{l}\text { Control } \\
\text { Ri } \\
\text { R-SHGi } \\
\text { R-SHG-Ci }\end{array}$ & $\begin{array}{l}6: 5.8(1.5) \\
4: 6.5(1.0) \\
1: 6.0(0.0) \\
4: 7.0(0.0)\end{array}$ & $\begin{array}{l}9: 4.7(2.5) \\
4: 4.5(2.9) \\
2: 6.5(1.0) \\
5: 6.6(0.9)\end{array}$ & $\begin{array}{l}8: 6.5(0.9) \\
6: 6.2(1.6) \\
2: 6.0(2.0) \\
4: 6.5(1.0)\end{array}$ & $\begin{array}{ll}\mathrm{ns} & \\
\mathrm{ns} & \mathrm{ns}^{2} \\
- & \\
\mathrm{ns} & \mathrm{ns}^{2} \\
\end{array}$ \\
\hline $\begin{array}{l}\text { differences in } \\
\text { adherence } \\
\text { between } \\
\text { conditions per } \\
\text { quartile' }\end{array}$ & & ns & ns & ns & \\
\hline
\end{tabular}

1. Friedman paired samples test, change in adherence behavior in time, per intervention condition.

2. Friedman paired samples test, change in adherence behavior in time, with or without self-help guide.

3. Kruskal Wallis test comparing differences between conditions per quartile.

After adjustment for pretest weekly frequency of wet episodes, no significant differences in IQOL or IIQ-7 scores were found between conditions at either measurement. Since symptoms improved, mean IQOL scores also improved significantly for women in all conditions. IQOL scores increased significantly from a mean of 84.5 (SD 14.2) at pretest to a mean of $93.7(\underline{\text { SD }} 13.4 ; \underline{F}(3,81)=24.296, \underline{p}=$ $.001)$ at one year follow-up. Similarly, mean IIQ-7 scores improved significantly from 2.0 (SD 2.5) at pretest to 0.9 (SD $2.1 ; \mathrm{E}(3,82)=15.497, \mathrm{p}<.001)$ one year after therapy. This means that effective PFME therapy with or without extra health 
education decreased symptom distress and symptom impact significantly. Mean item scores indicated that women were hardly worried about symptoms anymore after therapy, and perceived little or no impact on daily life activities. No differences were found between women with stress-, urge or mixed UI in IQOL or IIQ-7 scores.

\section{Perceived improvement and satisfaction}

Perceived improvement and satisfaction with treatment outcomes at 12-month followup were not significantly different between intervention conditions. One year after therapy, twenty-nine women $(28.1 \%$ of $n=103)$ reported being dry, 44 women $(40.8 \%)$ perceived much improvement of symptoms, 20 women perceived little improvement (19.4\%), nine women found that they had remained the same, and two found that their symptoms had deteriorated since they had started PFME therapy. Seventy women $(68 \%)$ were very satisfied with the treatment effects on incontinence symptoms and toilet behavior, 14 women were a little satisfied, 11 were neutral and six women were dissatisfied. According to the judgment of the women, PFME therapy had no influence on their physical or mental health, social life, job related activities, or care activities, but 38 women ( $36.9 \%$ ) expressed that their self-esteem had improved.

Both women in the experimental conditions and the physiotherapists were very positive about the health education program as a whole. Physiotherapists highly appreciated the self-help guide, considered it a useful addition to their therapy, expected the guide to encourage adherence behavior and wanted to use the self-help guide with all future female incontinence patients. The counseling aspects appeared to be more or less a part of regular care already, although only physiotherapists in the RSHG-Ci had been instructed to implement structured oral counseling.

All women were very satisfied with the PFME therapy and with the contact they had with the physiotherapists. The health education program was highly appreciated as well. Most women had read the entire self-help guide and found the information credible, understandable, clear, and attractive. Women marked the self-help guide with an eight (on a ten-point scale) and over three quarters of them would use the guide again and would certainly recommend the program to others. The folder with sticker reminders was appreciated likewise, but few women had used it as intended. Over three quarters $(77 \%)$ of the women found that the information in the guide helped them to understand incontinence and had encouraged their adherence to the advice of the therapist. The model stories and tips were considered helpful in supporting adherence 
behavior, in contrast to the filling-in tasks and stickers. The latter were hardly used, or used by some women when explicit attention had been given to that type of reminder. Twenty-nine women $(39.2 \%)$ used the guide for a few months after therapy, and 23 women $(31.1 \%)$ used the guide now and then up to one year after therapy.

\section{DISCUSSION}

This study assessed the effectiveness of PFME therapy supplemented with a theorydriven health education program on therapy outcome and long-term adherence behavior. The health education interventions, and in particular the self-help guide alone, had no significant additional impact on symptom outcome, nor on adherence behavior. Intention to treat analyses did not change the results. However, the individual PFME therapy was very successful. Weekly frequency of wet episodes drastically decreased during therapy, and results were maintained during the one year follow-up. The symptom cure and improvement rates we found one year after therapy, including intention to treat data, exceed those found in other studies on physiotherapeutic PFME therapy as reported in reviews (Berghmans et al., 1998a and 2000; Bø, 1995a; HaySmith et al., 2001; Mouritsen et al., 1994; Mouritsen and Schiøtz, 2000).

Adherence behavior of the women in this study was higher than found in other studies during a one year follow-up (Ferguson et al., 1990; Janssen et al., 2001; Lagro-Janssen et al., 1992). An important finding of our study was that women with more frequent losses before and after therapy, more likely retained a higher adherence level one year after therapy, than women with less frequent losses. Thus, most women seem to have learned to adapt their adherence behavior to their symptoms. A similar adaptation pattern was found in the study of Burns et al. (1993), who argued that women with mild symptoms may not perceive their incontinence as a problem warranting sustained effort, while women with many daily losses recognize it as an ongoing problem and make a persistent effort as positive response occurs. As stated, adherence level was high, but it is unclear whether women in this study had fewer wet episodes because their previous adherence (between pretest and posttest) was high or whether women had high adherence levels although they were dry or had mild UI symptoms. In contrast to others (Bø and Talseth, 1996; Chen et al., 1999; Lagro-Janssen and Van Weel, 1998), we did not find that women with higher adherence levels had fewer wet episodes at follow-up. 
An interesting explanation for the lack of impact of the health education program was found in the process evaluation. According to the remarks of the physiotherapists, working with the standardized protocol checklist for PFME therapy had structured the content of their therapy sessions, compared to their treatment behavior in the past. Together with the fact that physiotherapists had to fill in a treatment form for every woman to evaluate treatment goals, it may be expected that evaluation moments and concurrent feedback during therapy have been structured as well. Our results thus suggest that having a standardized protocol checklist for PFME therapy covering all treatment aspects and evaluation moments, which is intensively guided by enthusiastic physiotherapists, seems most important for realizing long-term effects and optimal adherence behavior. In our study, every woman had such intensive oral guidance during PFME therapy. This might explain why the written health education program had no significant additional influence.

Some flaws of the present study should be considered though. A Hawthorne effect may have biased our findings (Cook and Campbell, 1979). All therapists in our study knew that they were in a trial evaluating the effectiveness of additional health education to regular PFME therapy. They may unintentionally have given more and structured attention to the regular patient education part of their therapies. Social desirability may also have played a role, but this was not assessed among physiotherapist. Among the participating women, social desirability scores were high $(80 \%)$, which might explain the reporting of high adherence levels. Nevertheless, social desirability scores were equal between conditions and would therefore have influenced all scores in the same direction. Moreover, an attempt was made to minimize social desirability by using a five-point scale in stead of a dichotomous scale in the diary question on adherence behavior, in which the first three options referred to reasons for non-adherence, the fourth to moderate adherence and the fifth to optimal adherence. Another possibility is that the measurement of adherence itself could have functioned as a reminder for adherence behavior (Beurskens et al., 1992; Myers and Midence, 1998; Windsor et al., 1994), thereby significantly raising adherence levels in all conditions. If so, then it may be expected that the reminder phone calls for sending back the measurements would also have raised adherence levels (Burns et al., 1993; Nygaard et al., 1996). However, this was checked and no significant correlation was found between the number of calls per person and adherence behavior. Memory bias is not likely to have played a role in this study, because diaries are expected to prevent memory bias (Windsor et al., 1994). 
In studies on PFME therapy, adherence is most often associated with doing PFMEs, seldom with bladder training and never with the functional use of pelvic floor muscles in daily life, nor is it operationalized into such specific behaviors. Actual adherence behavior remains a black box, when only assessing how many days per week women follow behavioral advice or, for example, when only assessing how many repetitions of contractions women have performed. We tried to open this black box by asking additional information about adherence activities as presented in Table 1 . Unfortunately, this information turned out not reliable enough because of missing values or indistinct answers. Clearly, further studies on the assessment of adherence behavior are warranted.

We had anticipated to bring about a $25 \%$ larger relative reduction in symptoms when the control condition had improved by $50 \%$ at one year follow-up (Lagro-Janssen et al., 1992; Mouritsen, 1994). The fact that we had much fewer women in the study than we had planned to have on the basis of the power analysis, was not the problem, as we would still have found the expected difference with a power of $79 \%$. Furthermore, relative to other studies in this field, we were still able to include a large number of women in our study up to the last assessment at one year after therapy (Hay-Smith et al., 2001). It may be that it is much more difficult to reduce symptoms even further when they are already low after therapy (floor effect), which is reflected in the effects remaining stable after posttest, regardless of high adherence levels. In clinical terms, when women have improved very much after therapy, it is much more difficult to improve even further, which may explain why the health education program had no additional long-term effect. Average clinical improvement then could be seen as close to optimal.

With respect to the external validity, some comments should be made. Women were first recruited on the basis of risk factors for UI, and those with symptoms of UI were asked to participate in the study. This recruitment procedure was probably responsible for the fact that the general health status and co-morbidity of women in our study was slightly worse than that of the general population of women with UI. However, comorbidity is often seen in women with UI, especially in older women (Resnick, 1995), so our population might also reflect the true heterogeneous picture of women with UI seen in clinical practice (Mouritsen and Schiøt, 2000). In addition, a lot of the recruited women in our study did not want to participate because their symptoms did not bother them so much to start therapy as yet. Thus, the women who wanted to participate were probably highly motivated, which may explain their high adherence 
levels. This may point towards selection bias. However, that is a problem that all studies in this field encounter.

\section{Implications for practice}

Although we lack real proof whether the protocol checklist for PFME therapy (developed as a standard for all conditions) had optimized usual care, opinions of physiotherapists particularly confirmed that they worked more planned and systematically when using the protocol checklist. To reveal whether a structured therapy protocol such as used in our study really optimizes effectiveness of PFME therapy, a randomized controlled trial should be conducted to compare PFME therapy with checklist protocol to a control condition of PFME therapy without checklist protocol. The strongest argument in favor of considering implementation of the protocol checklist in existing practice guidelines for physiotherapeutic PFME therapy (see e.g. Berghmans et al., 1998a; Messelink et al., 2000), is that applying the protocol requires little investment, is easy to use and provides good insight into the activities of the physiotherapist. The use of the protocol can also be trained in the follow-up courses on pelvic floor dysfunctions.

Physiotherapists in our study found that the frequency of treatment sessions over time should remain individually targeted, which had not been the case in this study for standardization purposes. However, the number of treatment sessions in our study had reflected usual care, and had been patient-centered. This means that our optimal results were found with a therapy of normal length, which was prolonged only for those women who could benefit further from additional sessions. As the guidance and oral counseling by the physiotherapist seems the most important factor for success, further optimization of treatment outcome and adherence behavior may be realized by providing long-term follow-up treatment sessions for those women who continue to have bothersome symptoms and are in need of extra counseling. Practically, this can be established by including extra follow-up treatment sessions. Further research should evaluate the effectiveness of long-term follow-up treatment sessions, and determine at which moments they could best be provided. In addition, the organizational and financial consequences should be evaluated as well, in cooperation with paramedical organizations and insurance companies.

Nationwide implementation of the health education program in the form of a self-help guide with sticker-reminders does not seem warranted as it does not improve clinical 
outcomes when using it together with a standardized protocol checklist, and the effect on adherence behavior remains unclear. However, process evaluation revealed that these 28 physiotherapists would like to continue to work with the guide, and most women in the study had appreciated the information very much and would recommend it to others. We feel that this possitive response can not only be explained by possible social desirability of answers of both physiotherapists and women. Therefore, the possibility of implementation might be considered. Process evaluation findings also offered recommendations for the adaptation of the guide: leave more place for personal notes; diary pages on which women can write the behavioral or exercise advice of the therapist after each session should be replaced by texts and drawings of the most important exercises, or better, physiotherapists should give the personal behavioral and exercise advice on paper for every women appropriate to her situation.

To conclude, the results of this study show that symptoms of UI drastically reduced after individual PFME therapy relative to the severity of symptoms before therapy, and remained stable up to one year follow-up. This means that health professionals such as GPs, can tell their female patients with symptoms of UI that success can be expected when they invest sustainable time and effort in PFME therapy and adherence behavior. 


\section{CHAPTER 6}

\section{General discussion}

This thesis describes a study on the development, implementation and evaluation of a theory-driven health education program to promote long-term adherence to physiotherapeutic pelvic floor muscle exercise (PFME) therapy among women with stress-, urge- and mixed urinary incontinence (UI). The study assessed relevant predictors of long-term adherence to PFME therapy and evaluated the effectiveness of physiotherapeutic PFME therapy supplemented with a three component health education program on therapy outcome and adherence behavior in a longitudinal randomized controlled trial. In this chapter, main findings of the study are described, methodological issues are discussed, future research questions are proposed and implications for practice are suggested.

\section{Main findings}

The first aim of the study was to assess predictors of intention to adhere to PFME therapy at the onset of therapy (Chapter 3). Women who had large amounts of urinary loss per wet episode and women who had positive expectations about their ability to adhere to PFME therapy recommendations under various circumstances, had a positive intention to adhere to PFME therapy before the start of therapy.

After the intervention period, first the question had to be answered to what extent the implementation of the program components had been successful. Process evaluation revealed that study groups were less distinct with respect to the intervention components as planned. Namely, 1) few women in all experimental groups had used the stickers as reminders, and 2) the oral counseling aspects appeared to be equally applied in all study groups. The physiotherapists of the R-SHG-Ci group had received the counseling scheme with instructions, had been trained in a two-hour session and were asked to read an article on how to apply the key aspects of counseling. It may be that a more intensive training is required when specific counseling tasks have to be learned to systematize the commonly given patient education part of treatment (see e.g. Kerssens et al., 1999), or it may be that all physiotherapists already were equally and sufficiently qualified with respect to counseling skills. 
Either way, this means that in this study, having had a self-help guide remained the only distinction between the study groups.

The second aim of the study was to assess predictors of long-term adherence to PFME therapy (Chapter 4). Total explained variance of long-term adherence behavior one year after PFME therapy was fairly high, but of all determinants identified in the needs assessment, only a few appeared to be significant predictors of long-term adherence behavior. Women who were convinced that they were able to follow the behavioral advice of the physiotherapist under various circumstances, women who had a positive intention to adhere to PFME therapy recommendations and women who had frequent weekly wet episodes before and one year after therapy, had high adherence levels up to one year after therapy. In addition, short-term adherence immediately after therapy significantly predicted long-term adherence behavior, which may point towards habituation. In contrast to our expectations, the self-help guide was no significant predictor of long-term adherence behavior.

The third aim of the study was to evaluate the effectiveness of PFME therapy supplemented with the health education program compared to PFME therapy alone, on therapy outcome (Chapter 5). The health education interventions, and in particular the self-help guide alone, had no significant additional impact on therapy outcome. However, the individual PFME therapy was very successful in this respect. Incontinence symptoms drastically decreased from an average of 23 wet episodes per week to eight losses per week after therapy, and results were maintained during the one year follow-up. The symptom cure and improvement rates we found one year after therapy, including intention to treat data, exceed those found in other studies on physiotherapeutic PFME therapy.

Adherence behavior of the women in this study was also higher than found in other studies during a one year follow-up. Most women followed the behavioral advice on average six days per week at posttest and still four to five days one year after therapy. An important finding of our study was that women with more frequent losses before and after therapy, more likely remained a higher adherence level one year after therapy, than women with less frequent losses. Thus, most women seem to have learned to adapt their adherence behavior to their symptoms. As stated, adherence level was high, but it is unclear whether women in this study had fewer wet episodes because their previous adherence (between pretest and posttest) was high or whether 
women had high adherence levels although they were dry or had mild UI symptoms. In contrast to our expectations, we did not find that women with higher adherence levels had fewer wet episodes at follow-up.

An interesting explanation for the lack of impact of the health education program was found in the process evaluation. According to the remarks of the physiotherapists, working with the protocol checklist for PFME therapy (developed as a standard for all conditions) had structured the content of their therapy sessions compared to their treatment behavior in the past. Together with the fact that physiotherapists had to fill in a treatment form for every woman to evaluate treatment goals, it may be expected that evaluation moments and concurrent feedback during therapy have been structured as well. Our results thus suggest that having a standardized protocol checklist for PFME therapy covering all treatment aspects and evaluation moments, which is intensively guided by enthusiastic physiotherapists, seems most important for realizing long-term effects and optimal adherence behavior. In our study, every woman had such intensive oral guidance during PFME therapy. This might also explain why the written health education program had no significant additional influence to the individual guidance of PFME therapy.

\section{Methodological issues}

Several methodological issues of the study should be taken into account when interpreting the findings. Methodological issues discussed here are the power of the study, the representativeness of the study population, and the assessment of adherence behavior.

The first issue is the question whether we would have been able to detect a significant difference in therapy outcome between women in the intervention conditions compared to women in the control condition, when we would have had the number of women we had planned to have in the study on the basis of the power analysis. The fact that we had much fewer women in the study was not the problem, as we would still have found the expected difference with a power of $79 \%$. Furthermore, relative to other studies in this field, we were still able to include a large number of women in our study up to the last assessment at one year after therapy in each study condition (HaySmith et al., 2001). It may be that it is much more difficult to reduce symptoms even 
further when they are already low after therapy (floor effect), which is reflected in the effects remaining stable after posttest, regardless of high adherence levels. In clinical terms, when women have improved very much after therapy, it is much more difficult to improve even further, which may explain why the health education program had no additional long-term effect. Average clinical improvement then could be seen as close to optimal.

The second issue concerns the external validity of the study. Can the results of this study be generalized to women with UI in general? Several comments should be made. Because of the knowledge that most women don't seek help themselves, women were first recruited on the basis of risk factors for UI, and those reporting symptoms were asked to participate in the study. This recruitment procedure was probably responsible for the fact that the general health status and co-morbidity of women in our study was slightly worse than that of the general population of women with UI. However, comorbidity is often seen in women with symptoms of UI, especially in older women (Resnick, 1995), so our population might also reflect the true heterogeneous picture of women with UI seen in clinical practice (Mouritsen and Schiøtz, 2000). In addition, a lot of the recruited women in our study did not want to participate because their symptoms did not bother them so much to start therapy as yet. Thus, the women who wanted to participate were probably highly motivated. This may point towards selection bias. However, that is a problem that all studies in this field encounter.

The third issue concerns the measurement of adherence behavior. Among the women in this study, social desirability scores were high $(80 \%)$, which might explain the reporting of high adherence levels. Nevertheless, social desirability scores were equal between conditions and would therefore have influenced all scores in the same direction. Moreover, an attempt was made to minimize social desirability by using a five-point scale in stead of a dichotomous scale in the diary question on adherence behavior, in which the first three options referred to reasons for non-adherence, the fourth to moderate adherence and the fifth to optimal adherence. Another possibility is that the measurement of adherence itself could have functioned as a reminder for adherence behavior (Beurskens et al., 1992; Myers and Midence, 1998; Windsor et al., 1994), thereby significantly raising adherence levels in all conditions. If so, then it may be expected that the reminder phone calls for sending back the measurements would also have raised adherence levels (Burns et al., 1993; Nygaard et al., 1996). However, 
this was checked and no significant correlation was found between the number of calls per person and adherence behavior. Memory bias is not likely to have played a role in this study, because diaries are expected to prevent memory bias (Windsor et al., 1994). In addition, we tried to open the black box of actual adherence behavior by asking additional information about adherence activities, in terms of how many sessions and repetitions of pelvic floor muscle exercises women had performed per day and what other activities such as bladder training they had performed. Unfortunately, due to missing values and indistinct answers, this information turned out not reliable enough. Further research is needed to identify reliable and valid methods to assess adherence to PFME therapy.

\section{Implications for practice}

We conducted a pragmatic study in Dutch primary care to assess the surplus value of a health education program supplemented to usual care physiotherapeutic PFME therapy. What implications do our findings have for health educators and health professionals?

The prediction of long-term adherence to PFME therapy in this study was high. Remarkable is that, based on theory and empirical data, many possible determinants of adherence had been identified in the needs assessment. However, only a few appeared to be significant predictors of adherence behavior. Of these predictors, self-efficacy expectations and intention may be influenced through patient education. But, the selfhelp guide had no additional influence on these predictors, although it had included specific methods to enhance self-efficacy and stimulate a positive intention to adhere. Furthermore, the PFME therapy itself had no influence on self-efficacy either and intention had significantly decreased over time. Our results thus suggest that is is not necessary to address so many cognitive proximal and distal variables in a health education program. Furthermore, the high adherence levels we found may be explained by the motivating effect of the enthusiasm of the physiotherapists as well as the intensively guided therapy sessions.

In addition, although we lack real proof whether the standardized protocol checklist for PFME therapy had optimized usual care, opinions of physiotherapists particularly confirmed that they worked more planned and systematically when using the protocol checklist. To reveal whether a structured therapy protocol such as used in our study really optimizes effectiveness of PFME therapy, a randomized controlled trial should 
be conducted to compare PFME therapy with checklist protocol to a control condition of PFME therapy without checklist protocol. The strongest argument in favor of considering implementation of the protocol checklist in existing practice guidelines for physiotherapeutic PFME therapy (see e.g. Berghmans et al., 1998b; Messelink et al., 2000), is that applying the protocol requires little investment, is easy to use and provides good insight into the activities of the physiotherapist. The use of the protocol can also be trained in the follow-up courses on pelvic floor dysfunctions.

The optimal results in this study were found with a therapy of normal length, which was prolonged only for those women who could benefit further from additional sessions. As the guidance and oral counseling by the physiotherapist seems the most important factor for success, further optimization of treatment outcome and adherence behavior may be realized by providing long-term follow-up treatment sessions for those women who continue to have bothersome symptoms and are in need of extra counseling. Practically, this can be established by including extra follow-up treatment sessions. Further research should evaluate the effectiveness of long-term follow-up treatment sessions, and determine at which moments they could best be provided. In addition, the organizational and financial consequences should be evaluated as well, in cooperation with paramedical organizations and insurance companies.

Our findings and those of others (Dugan et al., 2001; Shaw et al., 2001) show that most women don't seek help because they don't regard their symptoms as a (medical) problem, and that many women lack knowledge about incontinence and treatment options. The results of this study also show that symptoms of UI drastically reduced after individual PFME therapy relative to the severity of symptoms before therapy, and remained stable up to one year follow-up. Thus, for health professionals, such as GPs, midwives, gynecologists or urologists, it is worth while to be aware of the potential of PFME therapy for women with UI. They may ask women whether they have symptoms of UI when this may be expected on the basis of well-known risk factors such as vaginal delivery, obesity, arthritis, asthma, a medical history of gynecological operations or increasing age (Diokno et al., 1990; Hannestad et al., 2001; Milsom et al., 1993, Milsom, 2000; Møller et al., 2000; Snook et al., 1990; Yarnell et al., 1982). Further studies should explore the attitudes of health professionals towards such active inquiry about symptoms of UI among women, in combination with providing patient education about treatment options. 
In addition, with respect to health education, there is a definite need for general mass media education on UI and management options, to make sure that women know what they can do when symptoms of UI start to bother them and they experience the need for treatment. Care should be taken, however, not to medicalize those who are not bothered enough by their symptoms and don't want treatment as yet. By all means, health professionals such as GPs, can tell their female patients with symptoms of UI, that success can be expected when they invest sustainable time and effort in PFME therapy and adherence behavior. 


\section{Summary}

Pelvic floor muscle exercise (PFME) therapy is recommended as first-choice treatment for women with stress-, urge- and mixed urinary incontinence. Short-term effectiveness is good. Up to $70 \%$ of treated women may be cured or much improved after therapy. However, long-term success rates are much lower $(40-50 \%)$ due to a decrease in adherence behavior. Clearly, there is a need for studies that identify relevant determinants of adherence to PFME therapy and develop and evaluate adherence-promoting programs.

This thesis describes a study on the development, implementation and evaluation of a health education program to promote long-term adherence to physiotherapeutic PFME therapy among women with urinary incontinence. The study assessed relevant predictors of long-term adherence to PFME therapy and evaluated the effectiveness of physiotherapeutic PFME therapy supplemented with a three component health education program on therapy outcome and adherence behavior. Main research question were: what are the relevant determinants of long-term adherence to PFME therapy among women with stress-, urge- and mixed incontinence; and, what is the surplus value of a health education program that is supplemented to usual care PFME therapy on therapy outcome and adherence behavior, one year after therapy? A longitudinal randomized controlled trial was conducted with a control condition and three intervention conditions. The control condition consisted of individual physiotherapeutic PFME therapy and the intervention conditions consisted of PFME therapy supplemented with one of the three health education interventions. PFME therapy in all conditions was standardized by means of a protocol checklist covering all treatment aspects, that reflected usual care. The 129 women included in the study completed self-administered questionnaires and diaries before therapy, immediately after therapy and at three and twelve months follow-up. Primary outcome measures were weekly frequency of wet episodes and adherence behavior.

The general introduction in Chapter 1 provides insight into the background of the study, the health problem urinary incontinence, effectiveness of PFME therapy, determinants of adherence to PFME therapy, and the study's design. Chapter 2 describes the development process of the health education program guided by the Intervention Mapping approach. First, a needs assessment based on empirical and theoretical data was conducted that identified many determinants of adherence behavior. It was apparent that these determinants were influenced by motivational readiness, knowledge and sex-specific aspects, and that there was no evidence that 
some determinants were more important than others. Adherence was operationalized in terms of the behavioral advice to performing 100 slow and fast twitch contractions during activities in five sessions per day, using sudden contractions to prevent leakage with coughing or urges, training the bladder when needed, and integrating the use of the pelvic floor muscles in the daily posture and movement pattern. For each determinant, specific adherence aims were formulated and appropriate methods and strategies were selected based on social cognition theory. The Transtheoretical model was applied to match educational messages to the changing information needs and motivational stages of women during PFME therapy. The Self-Regulation model was used because it provides insight into adherence as a self-management behavior. In addition, a checklist of twelve criteria for sex-specific health education guided targeting the information to women with urinary incontinence involved in PFME therapy.

A minimal, a medium and a maximum intervention were developed to be able to investigate what intensity of health education would be necessary to promote adherence effectively. This resulted in the development of three theory-driven health education interventions: a reminder intervention using stickers (Ri); a self-help guide intervention including the reminder (R-SHGi) to guide adherence as self-management process; and an intervention consisting of the reminder, the self-help guide and structured feedback in the form of oral counseling by the physiotherapist (R-SHG-Ci).

In Chapter 3 a cross-sectional analysis of pretest data is described. The ASE model was applied to explain the role of determinants of adherence to PFME therapy. The ASE model assumes that behavioral intention is determined by the three proximal variables, and that distal variables influence behavioral intention through the cognitive proximal variables. The needs assessment (Chapter 2) had revealed the following determinants. Proximal variables were positive and negative outcome expectations, social influences in terms of social support, social norms and modeling, and selfefficacy expectations. Distal variables included socio-demographic variables, (negative) experiences with sexuality, general health, daily life activities, self-esteem and body esteem.

Multiple regression analysis was employed to identify relevant predictors of intention to adhere to PFME therapy. At the onset of therapy, intention to adhere to PFME therapy was very positive. Two significant predictors of intention were identified. Large amounts of urinary loss per wet episode and positive self-efficacy expectations 
regarding adherence behavior, were related to a positive intention to adhere to PFME therapy before the start of therapy.

Chapter 4 describes the analyses of significant predictors of long-term adherence to PFME therapy. In addition, the evaluation of the effectiveness of the health education program supplemented to PFME therapy on adherence behavior and predictors of adherence is described. Sequential multiple regression analyses revealed several significant predictors, that together predicted up to $50 \%$ of variance in long-term adherence behavior. Positive intention to adhere, high short-term adherence levels and positive self-efficacy expectations significantly predicted high long-term adherence levels. Further, women with frequent weekly wet episodes before and after therapy, were more likely to have high adherence levels one year after therapy, than women with fewer losses. Thus, women seem to have learned to adapt long-term adherence behavior to their symptoms. The health education program had no additional influence on adherence behavior, nor on predictors of adherence.

Chapter 5 describes the process and effect evaluation of the study. Two parts of the health education program hadn't been implemented as planned: the reminder and the counseling intervention. Process evaluation further revealed that the self-help guide was used as planned and highly appreciated among physiotherapists and participants. The health education intervention, or the self-help guide alone, had no additional effect on treatment outcome. However, the individual PFME therapy was very successful. Weekly frequency of wet episodes drastically decreased from on average 23 wet episodes to eight losses, and results were maintained from posttest to one year followup. One year after therapy, $74.8 \%$ of the women $(n=103)$ were cured or improved by $50 \%$ (intention to treat $64.4 \%, \mathrm{n}=129$ ). Adherence behavior was very high with most women performing the exercise advice on average six days per week at posttest and four to five days one year after therapy. These results are higher than found in other studies.

Results suggested that the standardization protocol checklist for PFME therapy as used in this study, had resulted in such an optimalization of usual care in all conditions, that a written health education could not further improve therapy outcome or adherence behavior. According to the process evaluation conducted among physiotherapists, this written protocol had thus structurized therapy content. Furthermore, in order to evaluate treatment goals, physiotherapists had to complete treatment forms for every participating woman. Having to check accomplished treatment goals during each 
treatment, may also have stimulated physiotherapists to orally evaluate progress and subsequently provide reinforcement and feedback.

In Chapter 6 the main findings are described and discussed, and suggestions for further research and practice are proposed. It was suggested that existing practice guidelines for physiotherapeutic PFME therapy are supplemented with a standardized written protocol covering the most important treatment aspects and evaluation moments as a helpful tool. It was further suggested that health professionals tell their female patients with UI that success can be expected when they invest sustainable time and effort in PFME therapy and adherence behavior. 


\section{Samenvatting}

Oefentherapie van de bekkenbodemspieren wordt aanbevolen als eerste keuze van behandeling voor vrouwen met stress-, urge- of gemengde urine incontinentie. De korte termijn effectiviteit is goed. Tot $70 \%$ van de behandelde groep vrouwen is droog of verliest veel minder of minder vaak urine direct na de therapie. Het succes op lange termijn is echter minder goed, zo'n 40 tot $50 \%$, en dit hangt samen met een afname in de therapietrouw. Er is een duidelijke behoefte aan onderzoek naar de relevante determinanten van therapietrouw bij oefentherapie van de bekkenbodemspieren. Tevens is er behoefte aan onderzoek dat voorlichtingsprogramma's evalueert die gericht zijn op de bevordering van therapietrouw.

Dit proefschrift beschrijft een onderzoek naar de ontwikkeling, implementatie en evaluatie van een voorlichtingsprogramma dat gericht is op de bevordering van de lange termijn therapietrouw van vrouwen bij oefentherapie van de bekkenbodemspieren. In dit onderzoek werd onderzocht wat de relevante predictoren zijn van lange termijn therapietrouw bij oefentherapie van de bekkenbodemspieren. Tevens werd de effectiviteit onderzocht van oefentherapie van de bekkenbodemspieren aangevuld met een voorlichtingsprogramma bestaande uit drie componenten, op het urineverlies en de therapietrouw. De belangrijkste onderzoeksvragen waren: wat zijn de relevante determinanten van lange termijn therapietrouw bij oefentherapie van de bekkenbodemspieren bij vrouwen met stress-, urge of gemengde urine incontinentie, en wat is de meerwaarde van een voorlichtingsprogramma dat is toegevoegd aan de oefentherapie van de bekkenbodemspieren op het urineverlies en de therapietrouw één jaar na de therapie?

Om deze vragen te beantwoorden, werd een gerandomiseerd longitudinaal onderzoek uitgevoerd met een controle conditie en drie interventie condities. De controle conditie bestond uit individuele fysiotherapeutische oefentherapie van de bekkenbodemspieren en de interventie condities bestonden uit dezelfde therapie aangevuld met een van de drie voorlichtingsinterventies. De oefentherapie van de bekkenbodemspieren werd gestandaardiseerd door middel van een geschreven protocol bestaande uit een checklijst met de belangrijkste behandelaspecten. Dit protocol weerspiegelde zoveel mogelijk de gangbare zorg. De 129 vrouwen die participeerden in het onderzoek, vulden op vier momenten een vragenlijst en dagboek in: vlak voor de therapie begon, direct nadat de therapie was afgelopen en drie en twaalf maanden later. De primaire uitkomstmaten waren urineverlies uitgedrukt in de frequentie van het urineverlies per week en therapietrouw. 
De algemene inleiding in hoofdstuk 1 geeft inzicht in de achtergrond van de studie, het gezondheidsprobleem urine incontinentie, de effectiviteit van oefentherapie van de bekkenbodemspieren, determinanten van therapietrouw bij oefentherapie van de bekkenbodemspieren en de opzet van de studie. Hoofdstuk 2 beschrijft het ontwikkelingsproces van het voorlichtingsprogramma met behulp van Intervention Mapping. Uit een behoefte onderzoek, gebaseerd op zowel empirische als theoretische gegevens, bleek dat er veel verschillende determinanten van therapietrouw zijn. Het was duidelijk dat deze determinanten in belangrijke mate beïnvloed worden door de motivationele status, de aanwezige kennis en seksespecifieke aspecten, en tevens dat er geen bewijs was dat sommige determinanten belangrijker zijn dan andere. Therapietrouw werd geoperationaliseerd als de gedragsadviezen: het dagelijks uitvoeren van 100 snelle en langzame contracties van de bekkenbodemspieren gedurende dagelijkse activiteiten en verdeeld over vijf sessies, snelle contracties gebruiken om urineverlies te voorkomen bij hoesten of plotselinge sterke aandrang (urge), het trainen van de blaas indien nodig, en het integreren van het gebruik van de bekkenbodemspieren in het dagelijks bewegings- en houdingspatroon. Voor elke determinant werden vervolgens specifieke therapietrouwdoelen geformuleerd en daarbij werden passende methoden en strategieën geselecteerd, gebaseerd op principes van sociaal cognitieve theorieěn. Om de voorlichtingsboodschappen in het programma aan te laten sluiten bij de veranderende informatiebehoefte en motivationele stadia van vrouwen gedurende oefentherapie van de bekkenbodemspieren, werd het Transtheoretisch model gebruikt. De Self-Regulatie theorie werd gebruikt omdat deze inzicht geeft in therapietrouw als zelf-management gedrag. Tevens werd de voorlichting seksespecifiek gemaakt om de informatie zoveel mogelijk aan te laten sluiten bij vrouwen met ongewenst urineverlies die bezig zijn met oefentherapie van de bekkenbodemspieren.

Er werden drie interventies ontwikkeld, oplopend in intensiteit, om te kunnen onderzoeken welke intensiteit van voorlichting nodig zou zijn om de therapietrouw effectief te bevorderen. De drie op theorie gebaseerde voorlichtingsinterventies waren: een folder met geheugensteuntjes in de vorm van stickers (Ri); een zelfhulpgids met diezelfde stickers (R-SHGi) om therapietrouw als zelf-management gedrag te begeleiden; en een programma bestaande uit de stickers, de zelfhulpgids en gestructureerde feedback in de vorm van extra mondelinge voorlichting gegeven door de fysiotherapeut (R-SHG-Ci). 
In hoofdstuk 3 is een cross-sectionele analyse van de voormetingsdata beschreven. Het ASE model werd gebruikt om de rol van determinanten van therapietrouw bij oefentherapie van de bekkenbodemspieren te verklaren. Het ASE model gaat ervan uit dat gedragsintentie wordt bepaald door drie proximale variabelen, en dat distale variabelen de gedragsintentie beïnvloeden via de drie cognitieve proximale variabelen. Het behoefte onderzoek (hoofdstuk 2) had de volgende determinanten opgeleverd. Proximale variabelen waren positieve en negatieve uitkomstverwachtingen, sociale invloed in termen van sociale steun, sociale normen en modeling, en eigen effectiviteits verwachtingen. Distale variabelen waren socio-demografische variabelen, (negatieve) ervaringen met seksualiteit, algemene gezondheid, dagelijkse activiteiten, zelfbewustzijn en lichaamswaardering.

Met een meervoudige regressie analyse werden de relevante predictoren geỉdentificeerd van de intentie om therapietrouw te zijn bij oefentherapie van de bekkenbodemspieren. Bij aanvang van de therapie was de intentie om therapietrouw te zijn erg hoog. Twee significante predictoren werden gevonden. Het bleek dat hoe meer urine vrouwen verloren per keer, en hoe beter hun verwachtingen waren ten aanzien van de eigen effectiviteit met betrekking tot therapietrouwgedrag, hoe hoger hun intentie was om therapietrouw te zijn bij aanvang van de therapie.

Hoofdstuk 4 beschrijft de analyse van de significante predictoren van de lange termijn therapietrouw bij oefentherapie van de bekkenbodemspieren. Tevens beschrijft het de evaluatie van de effectiviteit van het voorlichtingsprogramma dat toegevoegd is aan de oefentherapie van de bekkenbodemspieren op de therapietrouw en predictoren van therapietrouw. Een stapsgewijze meervoudige regressie analyse bracht enkele significante predictoren aan het licht, die tot $50 \%$ van de variantie van de lange termijn therapietrouw konden verklaren. Een hoge therapietrouw één jaar na de therapie kon worden verklaard door een positieve intentie, een hoge therapietrouw direct na de therapie en positieve verwachtingen van de eigen effectiviteit. Verder hadden vrouwen die zowel voor als na de therapie vaker per week urine verloren een hogere therapietrouw dan vrouwen die minder vaak urine verloren. Dus het lijkt erop dat vrouwen hun therapietrouwgedrag aanpasten aan hun symptomen. Het voorlichtingsprogramma had geen additionele invloed op de therapietrouw, noch op de determinanten van therapietrouw. 
Hoofdstuk 5 beschrijft de effect- en procesevaluatie van de studie. Twee componenten van het voorlichtingsprogramma waren niet geïmplementeerd zoals gepland: het geheugensteuntje en de gestructureerde mondelinge voorlichting. De procesevaluatie liet verder zien dat de zelfhulpgids wel was gebruikt zoals gepland en dat deze erg werd gewaardeerd door zowel fysiotherapeuten als deelnemende vrouwen. De voorlichtingsinterventies, of de zelfhulpgids alleen, hadden geen additioneel effect op het urineverlies. Echter, de individuele oefentherapie van de bekkenbodemspieren was zeer effectief. De frequentie van het urineverlies per week was enorm gereduceerd van gemiddeld 23 keer tot acht keer, en de resultaten bleven na de eerste nameting stabiel tot één jaar na de therapie. De succespercentages verschilden dus niet tussen de condities, en één jaar na de therapie was $74.8 \%$ van de vrouwen $(n=103)$ droog of meer dan $50 \%$ verbeterd (inclusief uitvallers $64.4 \%, n=129$ ). De therapietrouw was erg hoog. Direct na de therapie voerden de meeste vrouwen de gedragsadviezen op gemiddeld zes dagen per week uit, en bleven dat zo'n vier tot vijf dagen doen een jaar na de therapie. Deze resultaten zijn hoger dan die van andere onderzoeken.

De resultaten wijzen erop dat de gestandaardiseerde protocol checklijst voor de oefentherapie van de bekkenbodemspieren, zoals gebruikt in deze studie, ervoor heeft gezorgd dat de gebruikelijke zorg in alle condities werd geoptimaliseerd, en dat het gebruik van een schriftelijk voorlichtingsprogramma de effecten van de therapie niet verder kon verbeteren. Volgens de procesevaluatie onder de fysiotherapeuten had het schriftelijke protocol de oefentherapie dus gestructureerd. Ook hadden de fysiotherapeuten voor elke deelneemster een behandelformulier met behaalde behandeldoelen ingevuld. Het zou goed kunnen zijn dat juist het bijhouden van de behaalde behandeldoelen, de fysiotherapeuten heeft gestimuleerd om vorderingen te evalueren en van mondelinge feedback te voorzien.

In hoofdstuk 6 worden de belangrijkste resultaten van het onderzoek beschreven en bediscussieerd, en worden aanbevelingen gedaan voor vervolgonderzoek en de praktijk. Er wordt voorgesteld om de bestaande richtlijnen voor oefentherapie van de bekkenbodemspieren uit te breiden met een gestandaardiseerd schriftelijk protocol dat de belangrijkste behandelaspecten bevat evenals evaluatiemomenten. Verder werd voorgesteld dat artsen hun vrouwelijke patiènten met ongewenst urineverlies kunnen vertellen dat succes kan worden verwacht als ze voldoende tijd en moeite steken in oefentherapie van de bekkenbodemspieren en in hun therapietrouw gedrag. 


\section{APPENDIX}

\section{Sex-specific health education}

An important tool to target health education messages is to make them sex-specific for four reasons. Sex differences are present in the nature and occurrence of illness and health that cannot only be explained by differences in biological factors. Furthermore, sex differences can be identified between the type of health problems, the use of health care resources, coping styles, the way of communication and the perception of illness and health. Taking these sex differences into account in health education practices in a way that the education links up with women's and men's perception of illness and health is expected to improve the quality of health education (Bertakis et al., 2000; Lagro-Janssen and Noordenbos, 1997; Stoverinck et al., 1996; Wagemakers and Van Bavel, 1998).

The ideas about sex-specific health education originated in a pilot project of the late Dutch Women's Health Care centre Aletta (Van Bavel and Wagemakers, 1996; Wagemakers and Van Bavel, 1998), and are based on the principles of the Women's Health Care movement which stem from the 1970's. The main principles of sexspecific health care are; 1) taking into account sex-specific aspects of illness and health such as prevalence differences, differences in the experience of illness and health between men and women and differences in the presentation of and coping with health problems; 2) explicitly paying attention to the relation between the social position and socialisation of women and men and illness and health; 3) stimulating autonomy and self-esteem of patients by being accessible, complete and recognizable; 4) reducing power imbalances in relations between health care provider and patients; 5) preventing medicalization, psychologizing and trivialization by offering alternatives and breaking down sex stereotypes; and 6) being open about the attitude and way of working while being aware of sex differences in communication styles (Lagro-Janssen and Noordenbos, 1997; Stuurgroep Vrouwenhulpverlening VWS, 1999; Van Bavel and Wagemakers, 1996; Wagemakers and Van Bavel, 1998).

Submitted, with some alterations as: Alewijnse, D., Bavel, M.A.P. van, Wagemakers, M.A.E. Seksespecifieke voorlichting: de vertaling naar de praktijk. 
In the health education program used in this study, this involved paying attention to determinants of adherence that were influenced by sex-specific aspects such as women's perception of their pelvic floor, to body- and self-esteem, to breaking down sex-stereotypes and medicalization, and to making explicit the relationship between women's socialization, social position and gender role and their adherence behavior (Janetzky, 1993; Toner and Akman, 2000).

In the above pilot project, the principles of sex-specific health care have been translated into twelve criteria for sex-specific health education (Wagemakers and Van Bavel, 1998). The twelve criteria of sex-specific health education enabled targeting the health education program to the target group of women with urinary incontinence (UI) involved in pelvic floor muscle exercise (PFME) therapy. The operationalization of the criteria was facilitated by questions guiding each criterium (Wagemakers and Van Bavel, 1998). Following, examples are given about the operationalization of each of these twelve sex-specific criteria in the written program, which for a large part consists of the self-help guide.

\section{The description of the target group of the health education is explicit about which group of women and/or men is being addressed.}

Although the target group is women with UI involved in PFME therapy which is made clear in the guide, prevalence figures are given for both men and women. This was done to break down the usual image that incontinence is a complaint of women. Furthermore, the different causes of incontinence for women and men are described. Differences in consequences of incontinence symptoms for men and women, however, are not worked out as the program is specific for women who are doing PFME therapy and also because such information about men is lacking.

\section{The sex-specificity of the health education is made explicit for the target group.}

It is made clear to whom the program guide is addressed: 'This guide has especially been written for women involved in PFME therapy. You have just started with this therapy which can be used for both men and women with stress-, urge- and mixed UI.' As the guide was solely written for women, no attention was payed to: differences between women and men in the prevalence of incontinence that would affect the content of the health education; different targets for women and men; the question whether possibilities to cope and deal with incontinence are different for women and men, nor to sex differences in choices for different treatments options. 
3. The health education is clear, understandable, comprehensible, reliable and attainable.

The goal of the health education is made clear: 'You can use this guide while doing PFME therapy as the text follows the content of the therapy. You can use it to write down the advised exercises so that you can read them over when you might have forgotten them or when you have relapsed from exercising.'

The guide is understandable and comprehensible because of the use of short sentences and words whenever possible. Furthermore, things are called by their name, thus 'vagina' instead of 'down under' which is often used. Difficult medical terms are avoided or explained.

The reliability of the guide is accomplished by describing the needs, experiences and questions of the target group in the form of three models being women of 34,51 and 76 years old. The text parts guiding the pictures of the models were derived from interviews in which women talked about daily life situations that make adherence to PFME therapy difficult. Stereotyping in the modeling was prevented by using one woman who was working and another woman who was not working, and the activities they were involved in were not merely activities normally associated with female roles in society. However, only white women were present as models as the guide was specifically tuned for white women and used as such in a research project in the southeast of The Netherlands. Knowledge on UI among other cultures is scarce, but it might be that for other cultures a different textual approach and totally different material, for example a videotape, would be more suitable than a self-help guide.

The attainability is accomplished by distribution of the guide through the physiotherapist. With respect to oral education, taking notice of the volume, intonation and tempo of the conversation as well as the use of body language and eye contact are important for the operationalization of this third criterium.

\section{The health education takes the patient seriously.}

To address this criterium, several questions guide the operationalization, such as:

Is there room and time for the ideas of the patients themselves?

Is the patient structurally involved in the health education, are the wishes and personal solutions of the patient being addressed and taken seriously?

Is there a collective definition of the health problem?

Are the experiences of the target group being respected and taken seriously? 
This criterium is hard to operationalize in text because it is open to various interpretations. The self-help guide encourages women to draw their own conclusions from factual information. This was stimulated through adding questions like 'How do you feel about this?' In addition, women are addressed directly in sentences like: 'Look for yourself how you experience this exercise and do what fits best to your feelings. If something bothers you, for example as doing pelvic floor muscle exercises elicit negative associations with sexuality or as they cause muscular pain, discuss this with your physiotherapist'.

To show that experiences are taken seriously, above mentioned models were used and room was given for different experiences in the text to show that not all women experience the same. Goal setting was used individually: 'Make your own goals by asking yourself what you want to be able to do without losing urine after PFME therapy.' This was also operationalized with the help of short assignments in the guide.

\section{The health education makes the relation between the health problem and the personal and social situation explicit.}

An example. The guide asks to think about the following questions: 'What is your personal and social situation? How can the behavioral advice be integrated in your daily life situation? What situations hamper following the behavioral advice of your physiotherapist, and are these easy to change?' For women who say that they are too busy with taking care of their jobs and their families so that no time and energy are left for themselves, the tip is given to take some private moments for themselves every day, rather than to focus on doing the exercises. The idea is that if women are able to make time for themselves, they can start to do exercises while doing something else, because doing that needs focussing on their own body for a while.

The relationship between women's socialization, norms and values about female roles in society and the way most women behave is explained, so that women can figger out if they themselves behave in a sex-stereotypic manner and whether they are bothered by that or not. Options for change are given in the form of tips. The interwovenness of biological, psychological and social health aspects according to incontinence problems is described in the chapter about daily consequences of incontinence episodes. Furthermore, the different beneficial and harmful coping strategies women often use, such as frequent voiding and restricting fluid intake, are made explicit. Hereby women can figger out what they need and want to change about their own behavior. 


\section{The health education takes into account the effect of imaging.}

What imaging about UI exists nowadays? How does this influence people's ideas about UI? Facts and myths stories were used to break down false ideas about UI, such as the myth that it is normal for women to become incontinent when growing old, or the myth that PFME therapy is only effective for young women. With this criterium the dilemma is the question whether models should be used that break through stereotypical roles by providing alternative behavioral options or stereotypical models to enlarge recognition by the target group. The self-help guide used both types of models. Furthermore, it was made explicit that advertisements about UI using older women might suggest that UI is a typical complaint of older women while the fact is that it is just a common complaint of adult women and older men and that it is more common in women.

\section{The health education makes the relation between the socialisation of men and women and health problems explicit.}

The influence of women's socialization on the difficulties with adherence to or experiences with PFME therapy are made explicit in the text and also with the use of the stories of the models. This was done for example according to the internalized caring duty and difficulty with adherence, and according to negative bodily experiences when doing PFME therapy which might be due to negative experiences with sexuality.

\section{The health education takes into account sex differences in communication.}

This criterium can be operationalized answering questions like:

What communication styles are being used in the health education materials and methods?

Is knowledge available about the effects of different communication styles on the target group?

The use of language (tone) and design of the self-help guide is targeted at women. For example by avoiding instrumental language, and by using stories with experiences of other women. In general, sex differences in communication may for example lead to chosing a different medium for an education message for women and men (Wagemakers et al., 2001). 


\section{The health education makes power relations explicit.}

Two questions can be asked to help operationalizing this criterium:

Is the health education explicit about the possible power imbalances between a health care provider and a patient, between men and women?

Does the health education break down inequality and discrimination?

As the guide is directed at the person herself and not so much at the interaction between the physiotherapist and the patient, this criterium was not operationalized in the guide. However, the practical clinical guideline for physiotherapists (Berghmans et al., 1998b) advises to promote a patient-centered approach in which an open atmosphere between physiotherapist and patient enables discussing treatment goals in cooperation with the patient. Thus, power imbalances between patient and physiotherapist are reduced as far as possible in PFME therapy itself.

\section{The health education is positive, stimulating and critical and breaks down matters of course. It encourages women and men to use their own power, possibilities, competencies and emotionality.}

The question is whether the health education focuses on the possibilities rather than on the impossibilities and whether the health education empowers and promotes autonomy and self-efficacy.

The challenge was to make the text of the program light without stepping over the real difficulties with adherence behavior as experienced by many women and without being too rosy. Therefore, as many tips as possible are given to promote adherence so that for every woman there are some useful options. Thereby, the importance to evaluate one's own progress is stressed and women are stimulated to be satisfied with every little goal they have reached. For example, women are advised to make a visualisation of some goal they want to reach after PFME therapy, for example doing sports without losing urine. Visualising goals is easier than thinking about goals because that's brain-work while using pelvic floor muscles correctly is a bodily experience that ultimately needs automatic and subconscious control.

Women's self-esteem and power are stimulated with reattribution like 'when you think that you aren't able to do the pelvic floor muscle exercises more often, think about when you succeeded in doing them.' Or, for women who are insecure about doing the exercises correct, women are learned to check for correct contractions themselves by, for example, inserting one or two fingers in their vagina and checking whether they can feel a contraction as well as asking their therapist to check their performance. 
The program stimulates women to think about what they experience as nice and what suits them best. It might even result in that they are not adherent and maintain using protective garments instead of adhere to PFME therapy recommendations.

\section{The health education informs about facts, opinions, feelings and experiences.}

The program has been developed in such a way that women could find appropriate answers to their own changing needs for information. Medical and psychosocial factors about UI are all mentioned in the program. For example, it is mentioned that UI is a symptom and not a disease, a symptom that something else is not OK, such as the pelvic floor or the bladder. The self-help guide further addresses various possible experiences and emotions or coping styles women may have because of symptoms of UI, so that women can recognize their own problems with UI and what causes these problems. The model stories play an important role in these messages.

\section{The health education gives different solutions and stresses people's own choices.} Necessary knowledge about the health problem, about relations between the health problem and daily life or about possible therapies is given, so that women can make their own choices for therapy. The positive and negative outcomes of other management options than PFME therapy are mentioned, but just objectively in figures so that women can decide for themselves what to do with them. A lot of possible tips for being adherent are mentioned from which women can choose what fits best to their personal situation. Pointed out is what women can do themselves and when it is good to talk with a doctor. Attention is given to mutual support groups and organisations where women can get information. Women are also stimulated to ask for information when necessary. Furthermore, addresses of organisations where information can be found and references of books about relevant subjects are listed in the guide.

This example of how to make health education sex-specific may help others in the field of health education in tuning their health education materials to women and men. Obviously, applying this criterialist enabled the development process of the health education program. However, some elements have to be drawn attention to. The criterialist for sex specific health education seems to be directed at individual and group education. However, sex specific aspects may also be put on the order-paper and for both sexes be explained in mass media health campaigns. 
Furthermore, not all criteria are adaptable to all health education situations. In addition, sometimes more knowledge is needed that is not yet available. For example, more insight is needed in the differences between men and women with regard to many health problems in order to elucidate what sex-specific health advice are most appropriate to men and women with a particular health problem (Editorial, 2001). In a broader perspective, in terms of taking into account diversity in health care as a whole, differences in, for example, culture, ethnicity, age or sexual preference, need as much attention as do sex differences in illness and health. 


\section{References}

Abrams, P., Blaivas, J.G., Stanton, S.L. and Andersen, J.T. (1988) The standardization of terminology of lower urinary tract function. Scandinavian Journal of Urology and Nephrology, (Suppl) 114, 5-19.

Ajzen, I. (1991) The theory of planned behavior. Organizational Behavior Human Decision Processes, 50, 179-211.

Ajzen, I. (2001) www.unix.oit.umass.edu/ ajzen.

Alewijnse, D. (1997) Oefentherapie van de bekkenbodemspieren. Praktische tips en informatie voor vrouwen die bezig zijn met oefentherapie van de bekkenbodemspieren. [Practical tips and information for women involved in pelvic floor muscle exercise therapy] Stereo \& Grafia, ISBN 90-73522-11-0.

Alewijnse, D., Mesters, I., Metsemakers, J., Adriaans, J., Borne, B. van den (2001) Predictors of intention to adhere to physiotherapy among women with urinary incontinence. Health Education Research, 16 (2), 173-186.

Alewijnse, D., Mesters, I., Metsemakers, J., Borne, B. van den. (accepted) Program development for promoting adherence during and after exercise therapy for urinary incontinence. Patient Education and Counseling.

Alewijnse, D., Mesters, I., Metsemakers, J., Borne, B. van den. (submitted) Predictors of long-term adherence to pelvic floor muscle exercise therapy among women with urinary incontinence.

Alewijnse, D., Metsemakers, J., Mesters, I., Borne, B. van den. (submitted). Effectiveness of pelvic floor muscle exercise therapy supplemented with a health education program to promote adherence among women with urinary incontinence.

Andersson, K.-E., Appell, R., Cardozo, L.D., Chapple, C., Drutz, H.P., Finkbeiner, A.., Haab, F., Navarrette, R.V. (1999) Review: The pharmacological treatment of urinary incontinence. Journal of Urology, 84, 923-947.

Ashworth, P.D. Hagan, M.T. (1993) Some social consequences of non-compliance with pelvic floor exercises. Physiotherapy, 79, 465-471.

Bandura, A. (1986) Social foundations of thought and action. Prentice Hall, Englewood Cliffs, NJ.

Bandura, A. (1997) The exercise of control. New York, Freeman.

Bartholomew, L.K., Parcel, G.S., Kok, G. (1998) Intervention Mapping: a process for developing theory- and evidence-based health education programs. Health Education and Behavior, 25, 545-563.

Bartholomew, L.K., Parcel., G.S., Kok, G., Gottlieb, N. (2000a) Intervention Mapping step 1: preparing matrices of proximal program objectives, in: Intervention Mapping: a process for designing theory- and evidence based health education and promotion programs. Mountain View, CA: Mayfield.

Bartholomew, L.K., Shegog, R., Parcel, G.S., Gold, R.S., Fernandez, M., Czyzewski, D.I., Sockrider, M.M., Berlin, N. (2000b) Watch, Discover, Think and Act: a model for patient education program development. Patient Education and Counseling, 39, 253 268.

Bartholomew, L.K., Parcel, G.S., Kok, G., Gottlieb, N.H. (2001) Intervention Mapping: designing theory and evidence based promotion programs. First Edition. Mayfield, Mountain View, California. ISBN 0-7674-1278-8.

Bassett, S.F., Petrie, K.J. (1999) The effect of treatment goals on patient compliance with physiotherapy exercise programmes. Physiotherapy, 85 (3), 130-137. 
Bekker, M. (1993) Handleiding van 'De Autonomie-lijst'. [Manual for the Autonomy Questionnaire] Lisse: Swets en Zeitlinger b.v.

Berghmans, L.C.M., Frederiks, C.M.A., De Bie, R.A., Weil, E.H.J., Smeets, L.W.H., Van Waalwijk van Doorn, E.S.C. and Janknegt, R.A. (1996) Efficacy of biofeedback, when included with pelvic floor muscle exercise treatment, for genuine stress incontinence. Neurourology and Urodynamics, 15, 37-52.

Berghmans, L.C.M., Hendriks, H.J.M., Bø, K., Hay-Smith, E.J., De Bie, R.A. and Van Waalwijk van Doorn, E.S.C. (1998a) Conservative treatment of stress urinary incontinence in women: a systematic review of randomized clinical trials. Britisch Journal of Urology, 82, 181-191.

Berghmans, L.C.M., Bernards, A.T.M., Hendriks, H.J.M., Bø, K., Grupping, M.H.M. (1998b) Guidelines for the physiotherapeutic management of genuine stress incontinence. Physical Therapy Reviews, 3, 133-147.

Berghmans, L.C.M., Hendriks, H.J.M., Bie, R.A. de, Van Waalwijk Van Doorn, E.S.C., Bø, K., Kerrebroeck, Ph.E.V. van. (2000) Conservative treatment of urge urinary incontinence in women: a systematic review of randomized clinical trials. Britisch Journal of Urology, 85, 254-263.

Bertakis, K.D., Azari, R., Callahan, E.J., Robbins, J.A. (2000) Gender differences in the utilization of health care services. The Journal of Family Practice, 49 (2), 147-152.

Beurskens, A.J.H.M., Bouter, L.M., Heijden, G.J.M.G. van der (1992) Compliance-bepaling bij oefentherapie. Een beoordeling van de beschikbare meetinstrumenten. [Assessing compiance with exercise therapy. A review of the available instruments). Nederlands Tijdschrift voor Fysiotherapie, 102 (1), 2-7.

Black, N.A., Downs, S.H. (1996) The effectiveness of surgery for stress incontinence in women: a systematic review. Britisch Journal of Urology, 78, 497-510.

Blaivas, J.G., Appell, R.A., Fantl, J.A., Leach, G., McGuire, E.J., Resnick, N.M., Raz, S., Wein, A.J. (1997) Definition and Classification of urinary incontinence: recommendations of the urodynamic society. Neurourology and Urodynamics, 16, 149-151.

Blaivas, J.G. (1998) Outcome measures for urinary incontinence. Urology, 51 (suppl. 2A), 1119.

Blowman, C., Pickles, C., Emery, S., Creates, V., Towell, L., Blackburn, N., Doyle, N. and Walkden, B. (1991) Prospective double blind controlled trial of intensive physiotherapy with and without stimulation of the pelvic floor in treatment of genuine stress incontinence. Physiotherapy, 77, 661-665.

Bø, K. (1995a) Review article: Pelvic floor muscle exercise for the treatment of stress urinary incontinence: an exercise physiology perspective. International urogynecology journal and pelvic floor dysfunction, 6, 282-291.

Bø, K. (1995b) Adherence to pelvic floor muscle exercise and long-term effect on stress urinary incontinence, a five-year follow-up study. Scandinavian Journal of Medicine, Science and Sports, 5, 36-39.

Bø, K., Hagen, R.H., Kvarstein, B., Jergensen, J. and Larsen, S. (1990) Pelvic floor muscle exercise for the treatment of female stress urinary incontinence: III. Effects of two different degrees of pelvic floor muscle exercises. Neurourology and Urodynamics, 9, 489-502.

Bø, K. and Talseth, T. (1996) Long-term effect of pelvic floor muscle exercise 5 years after cessation of organized training. Obstetrics \& Gynecology, 87, 261-265. 
Bø, K., Talseth, T., Vinsnes, A. (2000a) Randomized controlled trial on the effect of pelvic floor muscle training on quality of life and sexual problems in genuine stress incontinent women. Acta Obstetricia et Gynecologica Scandinavica., 79, 598-603.

Bø, K., Berghmans, L.C.M. (2000b) Nonpharmacologic treatments for overactive bladder pelvic floor exercises. Urology 55 (suppl 5A), 7-11.

Bouter, L.M., Dongen, M.C.J.M. van (1995). Epidemiologisch onderzoek, opzet en interpretatie. [Epidemiological research, interpretation and set up]. Derde herziene druk. Houten: Bohn Stafleu Van Loghum.

Brownell, K.D., Marlatt, G.A., Lichtenstein, E., Wilson, G.T. (1986) Understanding and preventing relapse. American Psychologist, 41 (7), 765-782.

Brownell, K.D., Cohen, L.R. (1995) Adherence to dietary regimens 2: components of effective interventions. Behavioral Medicine, 20, 155-164.

Brus, H., Laar, M. Van de, Taal., E., Rasker, J., Wiegman, O. (1997) Determinants of compliance with medication in patients with rheumatoid arthritis: the importance of self-efficacy expectations. Patient Education and Counseling, 36: 57-64.

Burgio, K.L., Robinson, J.C. and Engel, B.T. (1986) The role of biofeedback in Kegel exercise training for stress urinary incontinence. American Journal of Obstetrics and Gynecology, 154, 58-64.

Burgio, K.L., Engel, B.T. (1990) Biofeedback-assisted behavioral training form elderly men and women. Journal of the American Geriatric Society, 38, 338-340.

Burgio, L.K., Matthews, K.A., Engel, B.T. (1991) Prevalence, incidence and correlates of urinary incontinence in healthy, middle-aged women. The Journal of Urology, 146, 1255-1259.

Burgio, K.L., Locher, J.L., Goode, P.S. (2000) Combined behavioral and drug therapy for urge incontinence in older women. Journal of the American Geriatric Society, 48, 370374.

Burns, P.A., Pranikoff, K., Nochajski, T., Desotelle, P.H. and Harwood, M.K. (1990) Treatment of stress urinary incontinence with pelvic floor exercises and biofeedback. Journal of the American Geriatric Society, 38, 341-344.

Burns, P.A., Pranikoff, K., Nochajski, T.H., Hadley, E.C., Levy, K.J. and Ory, M.G. (1993) A comparison of effectiveness of biofeedback and pelvic muscle exercise treatment of stress incontinence in older community-dwelling women. Journal of Gerontology, 48, M167-M174.

Cammu, H., Van Nylen, M., Derdre, M-P., Debruyne, R., Amy, J-J. (1991) Pelvic physiotherapy in genuine stress incontinence. Urology, 38 (4), 332-337.

Cammu, H., Van Nylen, M. (1994) Pelvic floor muscle exercises: 5 years later. Urology, 45, 113-118.

Cammu, H., Van Nylen, M., Amy, J.J. (2000) A 10-year follow-up after Kegel pelvic floor muscle exercises for genuine stress incontinence. British Journal of Urology, 85, 655658.

Castleden, C.M Duffin, H.M. and Mitchell, E.P. (1984) The effect of physiotherapy on stress incontinence. Age and Ageing, 13, 235-237.

Chen, H-Y., Chang, W-C., Lin, W-C., Yeh, L-S., Hsu, T-Y., Tsai, H-D., Yang, K-Y. (1999) Efficacy of pelvic floor rehabilitation for treatment of genuine stress incontinence. Journal of the Formosan Medical Association, 98, 271-276.

Clark, N.M., Dodge, J.A. (1999) Exploring self-efficacy as a predictor of disease management. Health Education and Behavior, 26 (1), 72-89. 
Conner, M., Norman, P. (1995) The role of social cognition models in predicting health behaviours: future directions. In: Conner, M., Norman, P. (eds.) Predicting health behaviour. Research and practice with social cognition models. Open University Press, Buckingham.

Cook, Th.D., Campbell, D.T. (1979) Quasi-Experimentation. Design \& Analysis issues for field settings. Houghton Wifflin Company, Boston.

Cullen, K.W., Bartholomew, L.K., Parcel, G.S., Kok, G. (1998) Intervention Mapping: Use of theory and data in the development of a fruit and vegetable nutrition program for girl scouts. Journal of Nutrition Education, 30 (4), 188-195.

Cramer, J.A. (1995) Optimizing long-term patient compliance. Neurology, 45 (suppl 1): S25S28.

De Nooijer, J. (2001) Early detection of cancer. The development and evaluation of computertailored health education. Unigraphic, Thesis Maastricht University.

De Vries H., Dijkstra, M., Kuhlman, P. (1988) Self-efficacy: The third factor besides attitude and subjective norm as a predictor of behavioral intentions. Health Education Research, 3, 273-282

De Vries H., Mudde, A.N. (1998) Predicting stage transitions for smoking cessation applying the attitude-social influence-efficacy model. Psychology and Health, 13, 369-385.

DiMatteo, M.R., Reiter, R.C., Cambone, J.C. (1994) Enhancing Medication Adherence through communication and informed collaborative choice. Health Communication, 6 (4), 253-265.

Diokno, A.C., Brock, B.M., Herzog, A.R. and Bromberg, J. (1990) Medical correlates of urinary incontinence in the elderly, Urology, 36, 129-138.

Dishman, R.K. (ed.) (1994) Advances in exercise adherence. Edwards, US.

Dougherty, M., Bishop, K., Mooney, R., Gimotty, P., Williams, B. (1993) Graded pelvic muscle exercise, effect on stress urinary incontinence. The Journal of Reproductive Medicine, 38, 684-691.

Dubbelman, M.A.P. (2000) Onderzoek naar de kosteneffectiviteit van de diverse behandelvormen van stress-urine-incontinentie. [Cost-effectiveness of various treatments of stress urinary incontinence]. Projectgroep Ondernemingsstrategie en overheidsbeleid in de geneesmiddelensector. Erasmus Universiteit Rotterdam.

Dugan, E., Cohen, S.J., Bland, D.R., Preisser, J.S., Davis, C.C., Suggs, P.K., McGann P. (2000) The association of depressive symptoms and urinary incontinence among older adults. Journal of the American geriatric Society, 48, 413-416.

Dugan, E., Roberts, C.P., Cohen, S.J., Preisser, J.S., Davis, C.C., Bland, D.R., Albertson, E. (2001) Why older community-dwelling adults do not discuss urinary incontinence with their primary care physicians. Journal of the American Geriatrics Society, 49, 462-465.

Editorial (2001) Time for creative thinking about men's health. The Lancet, 357 (9271), 1813. Elia, G., Bergman, A. (1993) Pelvic muscle exercises: when do they work? Obstetrics \& Gynecology, 81, 283-286.

Ferguson, K.L., MeKey, P.L., Bishop, K.R., Kloen, P., Verheul, J.B. and Dougherty, M.C. (1990) Stress urinary incontinence: effect of pelvic muscle exercise. Obstetrics \& Gynecology, 75, 671-675.

Flynn, L., Cell, P., Luisi, E. (1994) Effectiveness of pelvic muscle exercises in reducing urge incontinence among community residing elders, Journal of Gerontological Nursing, 20 (5), 23-27. 
Franzoi, S.L., Shields, S.A. (1984) The body esteem scale: multidimensional structure and sex differences in a college population. Journal of Personality Assessment, 48, 173-178.

Franzoi, S.L. (1994) Further evidence of the reliabiliy and validity of the body esteem scale. Journal of Clinical Psychology, 50, 237-239.

Gallo, M.L., Staskin, D.R. (1997) Cues to action: pelvic floor muscle exercise compliance in women with stress urinary incontinence. Neurourology and Urodynamics, 16, 167 177.

Godin, G., Kok, G. (1996) The theory of planned behavior: a review of its applications to health-related behaviors. American Journal of Health Promotion, 11, 87-98.

Grealish, M., O'Dowd, T.C. (1998) General practitioners and women with urinary incontinence. British Journal of General Practice, 48, 975-978.

Green, L.W., Kreuter, M.W. (1991) Health promotion planning, an educational and environmental approach, second edition. Mayfield Publishing Company, Mountain View, California, London, Toronto.

Green, L.W., Kreuter, M.W. (1999) Health promotion and a framework for planning, in: Health promotion planning, an educational and ecological approach, third edition. Mayfield Publishing Company, Mountain View, California, London, Toronto.

Green, J. (2000) Editorial: The role of theory in evidence-based health promotion practice. Health Education Research, 15 (2), 125-129.

Hahn, I., Milsom, I., Fall, M. and Ekelund, P. (1993) Long-term results of pelvic floor training in female stress urinary incontinence. British Journal of Urology, 72, 421-427.

Hannestad, Y.S., Rortveit, G., Sandvik, H., Hunskaar, S. (2000): A community-based epidemiological survey of female urinary incontinence: The Norwegian EPINCONT study. Journal of Clinical Epidemiology, 53, 1150-1157.

Hay-Smith, E.J.C., Bø, K., Berghmans, L.C.M., Hendriks, H.J.M., de Bie, R.A., van Waalwijk van Doorn, E.S.C. (2001) Pelvic floor muscle training for urinary incontinence in women (Cochrane review). In: The Cochrane Library, Issue 1, 2001. Oxford: Update Software.

Health Council of the Netherlands; Urinary incontinence. The Hague: Health Council of the Netherlands, 2001; publication no. 2001/12.

Heitner, C.P. (2000) Valideringsonderzoek naar palpatie en myofeedback bij vrouwen met stress urine-incontinentie. [The validity of palpation and myofeedback among women with stress urinary incontinence]. Faculteit der Gezondheidswetenschappen, Bewegingwetenschappen, Universiteit Maastricht.

Holst, K., Wilson, P.D. (1988) The prevalence of female urinary incontinence and reasons for not seeking treatment. New Zealand Medical Journal, 101 (857), 756-758.

Holtedahl, K., Verelst, M., Schiefloe, A. (1998) A population based, randomized, controlled trial of conservative treatment for urinary incontinence in women. Acta Obstetricia et Gynecologica Scandinavica 77, 671-677.

Janetzky, C.R. (1993) Fysiotherapie en incontinentie. Tijdschrift voor Integrale Geneeskunde, 3, 270-276. (Physiotherapy and incontinence, Journal of Integral Medicine, The Netherlands).

Janssen, T., Miltenburg, T. (1998) Effectiviteit van oefentherapie bij incontinentie, individuele en groepsgewijze oefentherapie vergeleken. [Effectiveness of individual compared to group exercise therapy for incontinence]. Project Oefentherapie voor Vrouwen met Incontinentieklachten (PROVIN) i.s.m. Borghuis, M. Instituut voor Toegepaste Sociale Wetenschappen, Katholieke Universiteit Nijmegen. 
Janssen, C.C.M., Lagro-Janssen, A.L.M., Felling, A.J.A. (2001) The effects of physiotherapy for female urinary incontinence: individual compared with group treatment. British Journal of Urology International, 87, 201-206.

Janz, N.K., Becker, M.H. (1984) The Health Belief Model: a decade later. Health Education Quarterly, 11, 1- 47.

Jolleys, J.V. (1988) Reported prevalence of urinary incontinence in women in a general practice. British Medical Journal, 296, 1300-1302.

Johnson, T.M., Kincade, J.E., Bernard, S.L., Busby-Whitehead, J., DeFriese, G.H. (2000) Self-care practices used by older men and women to manage urinary incontinence: results from the national follow-up survey on self-care and aging. Journal of the American Geriatrics Society, 48, 894-902.

Joosten, J. and Drop, M.J. (1987) De betrouwbaarheid en vergelijkbaarheid van drie versies van de VOEG. [The reliability and validity of three versions of the VOEG-list]. Gezondheid en Samenleving, 4, 251-265.

Kegel, A.H. (1948) Progressive resistance exercise in the functional restoration of the perineal muscles. American Journal of Obstetrics and Gynecology, 56, 238-249.

Kerssens, J.J., Sluijs, E.M., Knibbe, J.J., Verhaak, P.F.M., Hermans, I.M.J. (1996) Het effect van therapietrouw verhogende strategieën in de fysiotherapie, patiënten van de controlegroep in hoofdlijnen. [The effect of adherence-promoting strategies in physiotherapy, patients of the control group in headlines]. Rapport Nivel, Utrecht.

Kerssens, J.J., Sluijs, E.M., Verhaak, P.F.M., Knibbe, H.J., Hermans, I.M.J. (1999) Educating patient educators: enhacing instructional effectiveness in physical therapy for low back pain patients. Patient Education and Counseling, 37 (2), 165-176.

Klarskov, P., Belving, D., Bischoff, N., Dorph, S., Gerstenberg, T., Okholm, B., Pedersen, P.H., Tikjøb, G., Wormslev, M. and Hald, T. (1986) Pelvic floor exercise versus surgery for female urinary stress incontinence. Urology International, 41, 129-132.

Knibbe, N.E., Wams, H.W.A. (1994) Met patiëntenvoorlichting methodisch werken aan therapietrouw, weten wat de patiënt beweegt. Nederlands Tijdschrift voor Fysiotherapie, 2, 44-51.

Kok, J., Bouter, L.M. (1990) Patiëntenvoorlichting door fysiotherapeuten in de eerste lijn. Nederlands Tijdschrift voor Fyiotherapie, 100 (2), 59-63.

Kok, G., De Vries, H., Mudde, A.N., Strecher, V.J. (1991) Planned health education and the role of self-efficacy: Dutch research. Health Education Research, 6, 231-238.

Kok, G.J., Bartholomew, L.K., Parcel, G.S., Gottlieb, N., Schaalma, H., Empelen, P. van (2000) Intervention Mapping: een protocol voor het ontwikkelen van op theorie en onderzoek gebaseerde gezondheidsvoorlichting. [Intervention Mapping: a protocol for the development of theory- and evidence based health education]. Tijdschrift voor Gezondheidswetenschappen, 2000, 78, 135-141.

Kreuter, M., Farrell, D., Olevitch, L., Brennan, L. (2000) Tailoring health messages, customizing communication with computer technology. Lawrence Erlbaum Associate Publishers, London. ISBN 0-8058-3387-0.

Kruif, Y.P. de, Wegen, E.E.H. van. (1996) Pelvic floor muscle exercise therapy with myofeedback for women with stress urinary incontinence: A meta-analysis. Physiotherapy, 82 (2), 107-113.

Laforge, R.G., Rossi, J.S., Prochaska, J.O., Velicer, W.F., Levesque, D.A. and McHorney, C.A. (1999) Stage of regular exercise and health-related quality of life. Preventive Medicine, 28, 349-360. 
Lagro-Janssen, A.L.M. (1991) Urine-incontinentie bij vrouwen in de huisartspraktijk. [Urinary incontinence among women in the general practice]. Medical Science. Thesis Catholic University Nijmegen.

Lagro-Janssen, A.L.M., Smits, A.J.A., Weel, C. van. (1990) Women with urinary incontinence: self perceived worries and general practitioners' knowledge of problem. British Journal of General Practice, 40, 331-334.

Lagro-Janssen, A.L.M., Debruyne, F.M.J., Smits, A.J.A. and Van Weel, C. (1992) The effects of treatment of urinary incontinence in general practice. Family Practice, 9, 284-289.

Lagro-Janssen, A.L.M., Smits, A.J.A., Van Weel, C. (1994) Gunstig effect van oefentherapie bij urine-incontinentie in de huisartspraktijk vooral afhankelijk van therapietrouw en motivatie. [Favorable effects of exercise therapy for urinary incontinence in the family practice especially dependent on adherence and motivation]. Nederlands Tijdschrift voor Geneeskunde, 138, 1273-1276.

Lagro-Janssen, A.L.M., Breedveldt Boer, H.P., Van Dongen, J.J.A.M., Lemain, T.J.J., Dijkstra, R.H.W., Wiersma, Tj. (1995) NHG-Standaard incontinentie voor urine. [Dutch Clinical Guideline on Urinary Incontinence for family physicians]. Nederlands Huisarts Genootschap M46. Huisarts en Wetenschap, 38, 71-80.

Lagro-Janssen, T., Noordenbos, G. (eds.) (1997) Sekseverschillen in ziekte en gezondheid. [Sex differences in illness and health]. SUN, Nijmegen.

Lagro-Janssen, T. and Van Weel, C. (1998) Long-term effect of treatment of female incontinence in general practice. British Journal of General Practice, 48, 1735-1738.

Lechner, E.H.S. (1998) Social psychological determinants of health risk behaviors related to cancer and CVD, applications and elaborations of the ASE model. Unigraphic, Thesis Maastricht University.

Lechner, L. and De Vries, H. (1995) Starting participation in an employee fitness program: Attitudes, social influences and self-efficacy. Preventive Medicine, 24, 627-633.

Lechner, L. and De Vries, H. (1997a) Nederlanders in de zon, risicogedrag en determinanten. [Dutch people in the sun, risk behavior and determinants]. Tijdschrift voor Sociale Gezondheidszorg, 61-69.

Lechner, L., De Vries, H., Offermans, N. (1997b) Participation in a breast cancer screening program: Influence of past behavior and determinants on future screening participation. Preventive Medicine, 26, 473-482.

Leventhal, H., Cameron, L. (1987) Behavioral theories and the problem of compliance. Patient Education and Counseling, 10, 117-138.

Leventhal, H., Leventhal, E.A., Contrada, R.J. (1998) Self-regulation, health and behavior: a perceptual-cognitive approach. Psychology and Health, 13, 717-733.

Lose, G., Fantl, J.A., Victor, A., Walter, S., Wells, T.L., Wyman, J., Mattiasson, A. (1998) Outcome measures for research in adult women with symptoms of lower urinary tract dysfunction. Neurourology and Urodynamics, 17, 255-262.

Mantle, J., Versi, E. (1991) Physiotherapy for stress urinary incontinence: a national survey. British Medical Journal, 302, 753-755.

Marcus, B.H., Owen N. (1992) Motivational readiness, self-efficacy and decision-making for exercise. Journal of Applied Social Psychology, 22, 3-16.

Marlatt, G.A., Gordon, J.R. (1985) Relapse Prevention. Maintenance strategies in the treatment of addictive behaviors. New York: The Guildford Press. 
Mattiasson, A., Djurhuus, J.C., Fonda, D., Lose, G., Nordling, J., Stöhrer, M. (1998) Standardization of outcome studies in patients with lower urinary tract dysfunction: A report on general principles of the International Continence Society. Neurourology and Urodynamics, 17, 249-253.

McAuley, E. (1993) Self-efficacy and the maintenance of exercise participation in older adults. Journal of Behavioral Medicine, 16, 103-113.

McGuire, W.J. (1985) Attitudes and attitude change. In: Lindzey, G., Aronson, E. (eds.) Handbook of Social Psychology, Volume II, 233-246. New York, Lawrence Erlbaum Associates.

Meichenbaum, D., Turk, D.C. (1987) Treatment adherence: terminology, incidence and conceptualization. In: Facilitating treatment adherence. A practitioner's guidebook. Plenum Press: New york and London.

Messelink, E.J. (1999) The overactive bladder and the role of the pelvic floor muscles. Britisch Journal of Urology International, 83 (suppl 2), 31-35.

Messelink, E.J., Hoope, C.A. ten, Lieshout, G.J.C.M. van, Peddemors, H., Slieker-Ten Hove, M.C.Ph., Weide, M.J.A. van der (2000) Consensus De overactieve blaas. [Consensus The overactive bladder]. Amsterdam.

Metsemakers, J.F.M., Hoppener, P., Knottnerus, J.A., Kocken, R.J.J. and Limonard, C.B.G. (1992) Computerized health information in the Netherlands: a registration network of family practices. British Journal of General Practice, 92,102-106.

Miller, W.R., Rollnick, S. (1991) Motivational Interviewing, preparing people to change addictive behavior. The Guilford Press, New York.

Miller, J.M., Ashton-Miller, J.A., DeLancey, J.O.L. (1998) A pelvic muscle precontraction can reduce cough-related urine loss in selected women with mild SUI. Journal of the American Geriatric Society, 46, 870-874.

Milsom, I. (2000) The prevalence of urinary incontinence. Acta Obstetricia et Gynecologica Scandinavica, 79, 1056-1059.

Milsom, I., Ekelund, P., Molander, U., Arvidsson, L. And Areskoug, B.J. (1993) The influence of age, parity, oral contraception, hysterectomy and the menopause on the prevalence of urinary incontinence in women. Urology, 149, 1459-1462.

Møller, L.A., Lose, G., Jørgensen, T. (2000) Risk factors for lower urinary tract symptoms in women 40 to 60 years of age. Obstetrics and Gynecology, 96, 446-451.

Moore, K.N., Dorey, G.F. (1999) Conservative treatment of urinary incontinence in men. A review of the literature. Physiotherapy, 85 (2), 77-87.

Morgan, D.L., Krueger, R.A. (1998) The focusgroup kit, volumes 1-6, Sage publications.

Morris, K. (1999) Tackling the taboo of urinary incontinence. The Lancet, 353, 128.

Mouritsen, L. (1994) Pelvic floor exercises for female stress urinary incontinence. International Urogynecology Journal, 5, 44-51.

Mouritsen, L., Frimodt-Møller, C., Møller, M. (1991) Long-term effect of pelvic floor exercises on female urinary incontinence. British Journal of Urology, 68, 32-37.

Mouritsen, L., Schiøtz, H.A. (2000) Pro et contra pelvic floor exercises for female stress urinary incontinence. Acta Obstetricia et Gynecologica Scandinavica, 79, 1043-1045.

Mullen, P.D., Green, L.W., Persinger, G.S. (1985) Clinical trials of patient education for chronic conditions: a comparative meta-analysis of intervention types. Preventive Medicine, 14, 753-781. 
Mullen, P.D., Simons-Morton, D.G., Ramirez, G., Frankowski, R.F., Green, L.G., Mains, D.A. (1997) A meta-analysis of trials evaluating patient education and counseling for three groups of preventive health behaviors. Patient Education and Counseling, 32, 157-173.

Murray, N., Kelder, S., Parcel, G., Orpinas, P. (1998) Development of an intervention map for a parent education intervention to prevent violence among hispanic middle school students. Journal of School Health, 68 (2), 46-52.

Myers, L.B., Midence, K. (1998) Methodological and conceptual issues in adherence, In: Myers, L.B., Midence, K. (eds) Adherence to treatment in medical conditions. Harwood Academic Publishers, Amsterdam.

Norman, P., Conner, M. (1995) The role of social cognition models in predicting health behaviours: future directions. In: M. Conner, P. Norman (Eds.), Predicting health behaviour: Research and practice with social cognition models. Buckingham: Open University Press.

Nutbeam, D. (1996) Achieving 'best practive' in health promotion: improving the fit between research and practice. Health Education Research, 11 (3), 317-326.

Nygaard, I., DeLancey, J.O.L., Arnsdorf, L., Murphy, E. (1990) Exercise and incontinence. Obstetrics \& Gynecology, 75, 848-851.

Nygaard, I.E., Kreder, K.J., Lepic, M.M., Fountain, K.A. and Rhomberg, A.T. (1996) Efficacy of pelvic floor muscle exercises in women with stress, urge, and mixed urinary incontinence. American Journal of Obstetrics and Gynecology, 174, 120-126.

Nygaard, I., Holcomb, R. (2000) Reproducibility of the seven-day voiding diary in women with stress urinary incontinence. International Urogynecology Journal and pelvic floor dysfunction, 11 (1), 15-17.

O'Brien, J., Austin, M., Sethi, P., O'Boyle, P. (1991) Urinary incontinence: prevalence, need for treatment, and effectiveness of intervention by nurse. British Medical Journal, 303, 1308-1312.

O'Dowd, T.C. (1993) Management of urinary incontinence in women. British Journal of General Practice, 43:426-429.

Payne, C.P. (2000) Behavioral therapy for overactive bladder. Urology 55 (suppl. 5A), 3-6.

Prochaska, J.O., DiClemente, C.C., Norcross. J.C. (1992) In search of how people change, applications to addictive behaviors. American Psychologist, 47, 1102-1114.

Prochaska, J.O., Velicer, W.F., Rossi, J.S., Goldstein, M.G., Marcus, B.H., Ranowski, W., Fiore, C., Harlow, L.L., Redding, C.A., Rosenbloom, D., Rossi, S.R. (1994) Stages of change and decisional balance for 12 problem behaviors. Health Psychology, 13 (1), 39-46.

Prochaska, J.O., Marcus, B.H. (1994) The Transtheoretical model: applications to exercise. In: Dishman, R.K. Advances in exercise adherence. Edwards, US.

Ramsay, L.N., Thou, M. (1990) Abstract 59: A randomised, double-blind, placebo controlled trial of pelvic floor exercises in the treatment of genuine stress incontinence. Neurourology and Urodynamics, 398-399.

Rekers, H., Drogendijk, A.C., Valkenburg, H., Riphagen, F. (1992) Urinary incontinence in women from 35 to 79 years of age: prevalence and consequences. European Journal for Obstetrics \& Gynecology and Reproductive Biology, 43, 229-234.

Resnick, N.M. (1995) Urinary incontinence. The Lancet, 346, 94-99. 
Sandvik, H., Seim, A., Vanvik, A., Hunskaar, S. (2000) A severity index for epidemiological surveys of female urinary incontinence comparison with 48-hour pad-weight tests. Neurourology and Urodynamics, 19, 137-145.

Seim, A., Sivertsen, B., Eriksen, B.C., Hunskaar, S. (1996) Treatment of urinary incontinence in general practice: observational study. Britisch Medical Journal, 312, 1459-1462.

Seim, A., Hunskaar, S. (2000) Female urinary incontinence - the role of the general practitioner. Acta Obstetricia et Gynecologica Scandinavica, 79, 1046-1051.

Shaw, C., Tansey, R., Jackson, C., Hyde, C., Allan, R. (2001) Barriers to help seeking in people with urinary symptoms. Family Practice, 18 (1), 48-52.

Sikkel, D. (1980) Een verkorting van de VOEG-schaal. [An abbreviation of the VOEG-list]. Sociaal Cultureel Kwartaalbericht, 2, 22-26.

Sluijs, E.M. (1991) Patient education in physical therapy. Thesis Maastricht University.

Sluijs, E.M. (1993) Therapietrouw door voorlichting, handleiding voor patiëntenvoorlichting in de fysiotherapie. [Adherence through education: Guideline for patient education in physiotherapy]. Reeks Patiëntenvoorlichting 6, LcGVO en Nivel, Utrecht.

Sluijs, E.M., Knibbe, J.J. (1991) Patient compliance with exercise: different theoretical approaches to short-term and long-term compliance. Patient Education and Counseling, 17, 191-204.

Sluijs, E.M., Kok, G.J., Van der Zee, J. (1993) Correlates of exercise compliance in physical therapy. Physical Therapy, 73 (11), 771-786.

Sluijs, E.M., Kerssens, J.J., Zee, J. Van der, Myers, L.B. (1998) Adherence to physiotherapy, In: Myers, L.B., Midence, K. (eds) Adherence to treatment in medical conditions. Harwood Academic Publishers, Amsterdam.

Snooks, S.J., Swash, M., Mathers, S.E., Henry, M.M. (1990) Effect of vaginal delivery on the pelvic floor: a 5-year follow-up. Britisch Journal of Surgery, 77, 1358-1360.

Steiner, J.F., Earnest, M.A. (2000) Lingua Medica: The language of medication-taking. Annals of Internal Medicine, 132 (11), 926-930.

Stoverinck, M.J.M., Lagro-Janssen, A.L.M., Weel, C. van (1996) Sex differences in health problems, diagnostic testing and referral in primary care. The Journal of Family Practice, 43 (6), 567-576.

Strecher, V.J., Seijts, G.H., Kok, G.J., Latham, G.P., Glasgow, R., DeVellis, B., Meertens, R.M., Bulger, D.W. (1995) Goal setting as a strategy for health behavior change. Health Education Quarterly, 22 (2), 190-200.

Stuurgroep Vrouwenhulpverlening Ministerie van Volksgezondheid, Welzijn en Sport (1999) Naar een seksespecifieke en multiculturele gezondheidszorg in de 21 ste eeuw. [Towards sex-specific and multicultural health care in the 21st century, Stearing committee of The Ministry of Public Health, Welfare and Sports]. BB\&A Grafische communicatie, Den Haag.

Tabachnick, B.G., Fidell, L.S. (1996) Using multivariate statistics. Third edition ed. Northridge: Harper Collins College Publishers.

Thomas, T.M., Plymat, K.R., Blannin, J., Meade, T.W. (1980) Prevalence of urinary incontinence. British Medical Journal, 281, 1243-1245.

Toner, B.B., Akman, D. (2000) Gender role and irritable bowel syndrome: literature review and hypothesis. The American Journal of Gastroenterology, 95 (1), 11-16. 
Uebersax, J.S., Wyman, J.F., Shumaker, S.A., McClish, D.K., Fantl, J.A. and Group CfWR. (1995) Short forms to assess life quality and symptom distress for urinary incontinence in women: the incontinence impact questionnaire and the urogenital distress inventory. Neurourology and Urodynamics, 14, 131-139.

Van Assema, P., Mesters, I., Kok, G. (1992) Het focusgroep-interview: een stappenplan. [The focusgroup-interview: a stepwise approach]. Tijdschrift voor Sociale Gezondheidszorg, 70, 431-437

Van Bavel, M., Wagemakers, A. (1996) Vrouwen \& Mannen \& Gezondheidsvoorlichting. De meerwaarde van een seksespecifieke benadering. [Women \& Men \& Health Education. The surplus value of a sexspecific approach]. Aletta, Drukkerij Den Daas, Utrecht

Van Campen, C., Sluijs, E.M. (1989) Bibliography patient compliance, a survey of reviews (1979-1989). NIVEL, Utrecht, The Netherlands.

Van Campen, C., Sixma, H., Kerssens, J.J., Peters, L. (1998) Comparisons of the costs and quality of patient data collection by mail versus telephone versus in-person interviews. European Journal of Public Health, 8 (1), 66-70.

Van den Berg, J. (1992) De gezondheidsenquete van het CBS. [The health-survey of the CBS]. In Gunning-Schepers, L.J. and Mootz, M. (eds), Gezondheidsmeting, reeks gezondheidsbeleid. Houten: Bohn Stafleu Van Loghum.

Van Dongen, J.J.A.M. (1998) Stress-incontinentie bij vrouwen ouder dan 55 jaar. [Stress incontinence among women older than 55 years]. Tijdschrift Huisartsgeneeskunde, 15 (6), 357-360.

Van der Vaart, C.H., Leeuw, J.R.J. de, Roovers, J.P.W.R., Heintz, A.P.M. (2000) De invloed van urine-incontinentie op de kwaliteit van leven bij thuiswonende Nederlandse vrouwen van 45-70 jaar. Nederlands Tijdschrift voor Geneeskunde, 144 (19), 894-897.

Verhulst, F.J.C.M., Van der Burgt, M.C.A., Lindner, K. (1994) Concretisering van patiëntenvoorlichting in het fysiotherapeutisch handelen. Nederlands Tijdschrift voor Fyiotherapie, 1, 10-17.

Versprille-Fischer, E.S. (1995) Begeleiding van patiënten met bekkenbodemdysfunctie. [Guidance of patients with pelvic floor dysfunctions]. Lemma, Utrecht.

Vierhout, M.E. (1990) Meting van ongewenst urineverlies bij de vrouw. [Assessment of urinary incontinence among women, Dutch Journal of Medicine]. Nederlands Tijdschrift voor Geneeskunde, 134, 1837-1840.

Visser, A., Breemhaar, B. (1989) Social desirability and program evaluation in health care. Impact Assessment Bulletin, 7, 99-112.

Wagemakers, A., Bavel, M. van (1998) Vrouwengezondheidszorg in de praktijk. GVO en preventie. [Women's Health Care in practice. Health Education and Prevention]. Aletta, Drukkerij Zuidam, Utrecht.

Wagemakers, M.A.E., Woerkum, C.M.J. van, Alewijnse, D. (2001) De factor sekse bij communicatiemodellen voor de gezondheidszorg. [Models on health communication and gender]. Tijdschrift voor gezondheidswetenschappen, 79 (3), 143-149.

Wagner, T.H., Patrick, D.L., Bavendam, T.G., Martin, M.L. and Buesching, D.P. (1996) Quality of life of persons with urinary incontinence: development of a new measure. Urology, 47, 67-72.

Wall, L.L., Davidson, T.G. (1992) The role of muscular re-education by physical therapy in the treatment of genuine stress urinary incontinence. Obstetrical and Gynecological Survey, 47, 322-331. 
Weijts, W., Van den Borne B., Metsemakers, J, Knottnerus, A. Risk factors for stress and mixed incontinence among women: a gender-sensitive approach. (Unpublished data).

Weiner, B. (1985) An attributional theory of achievement motivation and emotion. Psychological Review, 92 (4), 548-573.

Wells, T.J. (1990) Pelvic (Floor) Muscle Exercise. Journal of the American Geriatric Society, 38, 333-337.

Wells, T.J., Brink, C.A., Diokno, A.C., Wolfe, R. and Gillis, G.L. (1991) Pelvic muscle exercise for stress urinary incontinence in elderly women. Journal of the American Geriatric Society, 39, 785-791.

Willey, C., Redding, C., Stafford, J., Garfield, F., Geletko, S., Flanigan, T., Melbourne, K., Mitty, J., Jaime Caro, J. (2000) Stages of change for adherence with medication regimens for chronic disease: development and validation of a measure. Clinical Therapeutics, 22 (7), 858-871.

Wilson, P.D., Samarrai, T.A., Deakin, M., Kolbe, E., Brown, A.D.G. (1987) An objective assessment of physiotherapy for female genuine stress incontinence. Britisch Journal of Obstetrics and Gynaecology, 94, 575-582.

Windsor, R., Baranowski, T., Clark, N., Cutter, G. (1994) Evaluation of Health Promotion, Health Education and Disease Prevention Programs, second edition. Mayfield Publishing Company, Mountain View, California. ISBN:1-55934-243-9.

WVC, Ministerie (1994) De wet BIG, hoofdlijnen van de wet Beroepen in de Individuele Gezondheidszorg. [Headlines of the law for professionals in the individual health care]. Rijswijk.

www.cbs.nl, 2001. Website of Dutch Central Statistics Office.

Wyman, J.F., Choi, S.C., Harkins, S.W., Wilson, M.S., Fantl, J.A. (1988) The urinary diary in evaluation of incontinent women: a test-retest analysis. Obstetrics \& Gynecology, 71, 812-817.

Wyman, J.F., Harkins, S.W., Fantl, J.A. (1990) Psychosocial impact of urinary incontinence in the community-dwelling population. Journal of the American Geriatrics Society, 38, 282-288.

Wyman, J.F., Fantl, J.A., McClish, D.K., Bump, R.C. and the Continence Program for Women Research Group. (1998) Comparative efficacy of behavioral interventions in the management of female urinary incontinence. American Journal of Obstetrics and Gynecology, 179, 999-1007.

Yarnell, J.W.G., Voyle, G.J., Sweetman, P.M., Milbank, J., Richards, C.J., Stephenson, T.P. (1982) Factors associated with urinary incontinence in women. Journal of Epidemiology and Community Health, 36, 58-63. 


\section{Dankwoord}

Vele mensen hebben de afgelopen jaren een belangrijke bijdrage geleverd aan de ideevorming, het proces, de uitvoering, de verslaglegging en de afronding van dit onderzoeksproject en proefschrift. En het zijn die mensen die ik op deze plaats wil bedanken voor al hun inspanningen, vertrouwen, steun en enthousiasme.

Wie zijn dat en wat hebben zij gedaan?

De eerste ideeên voor het onderzoeksproject kwamen van Pauline Tomlow en Wies Weijts, die later door Wies, Bart van den Borne en André Knottnerus verder zijn uitgewerkt tot twee projectvoorstellen. De eerste was een prevalentiestudie naar het voorkomen van ongewenst urineverlies bij vrouwen met risicofactoren voor urineincontinentie en de tweede was dit onderzoeksproject, waaraan ook vrouwen uit de prevalentiestudie konden deelnemen.

De belangrijkste mensen die intensief betrokken zijn geweest gedurende heel het proces en van wie ik veel heb geleerd, zijn uiteraard de begeleiders: Wies Weijts, Ilse Mesters, Job Metsemakers en Bart van den Borne. Wies was het eerste jaar mijn dagelijks begeleidster met enorm veel enthousiasme, vrouwenstudies spirit en toewijding. Wies kreeg een andere baan en Ilse Mesters volgde haar in het tweede jaar op met minstens zoveel toewijding. Ik kon letterlijk altijd bij haar terecht, ze dacht met me mee en voor elk probleem dat we tegenkwamen had ze wel een paar praktische oplossingen. Ik zal nooit vergeten hoe we samen uren hebben zitten SPSS-en, uniek gewoon. Job als tweede dagelijks begeleider en Bart als promotor waren er het hele project bij. Job leerde me hoe je onderzoek kunt doen waarbij zowel huisartsen, fysiotherapeuten als patiënten betrokken zijn. Tevens zette hij kort, duidelijk en krachtig de noodzakelijke punten op de vele i's, waarbij hij telkens mijn wetenschappelijk denken prikkelde. Daarnaast zette hij me altijd weer met de benen op de grond met behulp van fantastische voorbeelden als ik weer eens door de bomen het bos niet meer zag. Bart bedacht in zijn enthousiasme voor het project soms nog vijf onderzoeksvragen erbij en dat hield de discussie en aanscherping levend. Ondertussen hield hij de rode draad in de gaten en dat leverde altijd weer nuttige opmerkingen op. Dankbaar denk ik terug aan alles wat ik van hen leerde en ik heb fijne herinneringen aan de vele intensieve voortgangsvergaderingen. 
Tijdens de voorstudie naar determinanten van therapietrouw hielden we groepinterviews met bekkenbodemtherapeuten: Mw. Y. Janss, Mw. M. Bos-Kessels, Mw. K. van der Kruis, Mw. C. Op 't Hof, Mw. J. Schurgers-Swaans, Mw. L. Smeets en Mw. N. Smit; en met vrouwen met ongewenst urineverlies al dan niet met ervaring met oefentherapie van de bekkenbodemspieren. Hen noem ik niet bij naam om privacyredenen. Bij de ontwikkeling van het voorlichtingsmateriaal hebben een aantal deskundigen een belangrijke bijdrage geleverd. Dat zijn op het gebied van seksespecifieke gezondheidszorg en -voorlichting: Mw. L. Nicolaï, Mw. E. Spuybroek en Mw. M.A.E. Wagemakers; en op het gebied van bekkenbodemtherapie: Mw. F. de Bruijne-Mouthaan, Mw. M. ten Brinke, Mw. M. Grupping, Mw. M. Raadgers, en bewegingstherapie: Mw. A. van Laar. Hetty Creemers en Nora Oosting zorgden uiteindelijk voor een mooie professionele vormgeving. Daarnaast wil ik ook de vrouwen bedanken die mee hebben gewerkt aan de pilotstudie van de meetinstrumenten, evenals hun bekkenbodemtherapeuten: Mw. M.C.A.Y. Halfens, Mw. J.M.C. Jaspars-Godfroy, Mw. C.T. Kerkhoffs, Mw. H.C.H.M.A. Klein Tuente, Mw. T. Maas, Mw. A.M.E. Maassen-Kock, Mw. M. Pepels v.d. Heuvel, Mw. M. Scheele, Mw. L. Smeets, Mw. N.H.M.M. van Thor en Mw. Weijnen-Dohmen.

$\mathrm{Na}$ deze voorbereidings- en ontwikkelingsfase kon de uitvoering van het onderzoek beginnen. De medewerkers Gregor Franssen en Alphons Schroten van het MEMIC, Centrum voor Data en Informatiemanagement, selecteerden nauwkeurig vrouwen met risicofactoren voor ongewenst urineverlies uit het databestand van dertien huisartspraktijken aangesloten bij het RegistratieNet Huisartspraktijken (RNH). Deze dertien huisartspraktijken verzorgden in 1998 vervolgens de werving en selectie van vrouwen met ongewenst urineverlies. Veel dank gaat dan ook uit naar de praktijkassistenten die alle administratieve handelingen hebben verricht en de huisartsen voor hun medewerking: Dhr. P. Castermans, Dhr. B. Huijnen, Mw. M. Maaskant, Dhr. B. Schilten, Dhr. P. Stalenhoef (Gezondheidscentrum Dr. van Kleef, Maastricht); Dhr. V. Kaiser, Dhr. J.M. Veldhuizen (Eygelshoven); Dhr. J. van der Ploeg (Kerkrade); Dhr. R.J.M.G. Costongs, Dhr. P.B.N. Hulshof, Dhr. R.A.M. Panhuysen (Voerendaal); Mw. B. Bruls, Dhr. P. van Dijk, Dhr. R. Helmers, Dhr. M. van Nunen, Dhr. H. Paas, Dhr. P. Voorhoeve (Gezondheidscentrum Hoensbroek Noord); Dhr. F. Guldemond, Mw. E.T.I.M. Guldemond-Hecker (Ubachsberg); Dhr. R.M.F.M. Leclercq, Dhr. J.P. van Proosdij (Stein); Dhr. J. Eussen, Mw. J.C. Smits, Mw. C. Stuurman, Dhr. H. Ypma (Medisch Centrum Putstraat, Landgraaf); Dhr. M. 
Op den Kamp (Heerlen); Dhr. J. Rinkens (Kerkrade); Dhr. B. Otten (Medisch Centrum, Merkelbeek); Dhr. A. Drost, Dhr. C. Hoogervorst, Dhr. W. Nieuwdorp (Huisartsenmaatschap Brunssum); Mw. A. Muysken, Dhr. G. Peeters en Dhr. F. Vissers (Gezondheidscentrum Heer, Maastricht).

Via hun huisarts ontvingen vele vrouwen een wervingsvragenlijst en werden uiteindelijk de 129 deelnemende vrouwen verwezen naar een fysiotherapeut voor oefentherapie van de bekkenbodemspieren. Gedurende een jaar vulden zij vier keer een behoorlijk lange vragenlijst en incontinentiedagboek in. Dat viel niet altijd mee en des te erkentelijk ben ik met hun medewerking aan het onderzoek. De bekkenbodemtherapeuten die de oefentherapie gaven met of zonder extra voorlichtingsmateriaal waren allemaal even enthousiast over het project, en dat heeft mij altijd enorm gestimuleerd. Veel dank gaat dan ook uit naar: Mw. J. Bijsterveld (Amstenrade); Mw. M. de Bruin, Mw. Y. Blokland-Lebens (Beek); Mw. C. BrouwersBeelen, Mw. M.J.H. Smeets (Beek); Mw. C. Aghina (Brunssum); Mw. P. Delnoy (Brunssum); Mw. E.J.A. Boer-Trimbos (Heerlen); Mw. R. Debets-Eggen (Heerlen); Mw. G.E.M. Persoon-Nievelstein (Heerlen); Mw. H.L.A.M. Robijns-Vreuls (Heerlen); Mw. L. Wald-Weischer (Heerlen); Mw. I. Willems-v.d. Cruijsen, Mw. E. van Neerven (Hoensbroek); Mw. M.M. Lauvenberg-Savelsberg (Kerkrade); Mw. C. BloklandVerhoeve, Mw. J. Klinkenberg (Landgraaf); Mw. E. Reinartz (Landgraaf); Mw. I. van Eijck (Maastricht); Mw. Y. Janss (Maastricht); Mw. I. Lacroix (Maastricht); Mw. C. Op 't Hof (Maastricht); Mw. M. Peters (Maastricht); Mw. J. Schurgers-Swaans (Maastricht); Mw. E. Wagner (Maastricht); Mw. A. van de Donk (Stein); Mw. M. BosKessels (Voerendaal) en Mw. M. van de Maas (Voerendaal).

Bij de organisatie, dataverwerking en het schrijven van hoofdstuk 3 ben ik bijgestaan door onderzoeksassistente Jeanne Adriaans. Het was heel fijn om een maatje te hebben die me veel werk uit handen nam tijdens die turbulente periode, en ook nu delen we nog lief en leed. Toen ik later zelf verder ging met data-invoer, kreeg ik al snel last van RSI en sprong ook Emily de Vet nog bij. Daarnaast gaat mijn dank uit naar de statistici die me van tijd tot tijd hebben geholpen de analyses te begrijpen: Dhr. T. Ambergen, Dhr. M. Berger en Dhr. H. Schouten. Bary Berghmans en Pytha Heitner dank ik voor hun steun en de nuttige discussies over dit onderzoeksproject en dat van henzelf, en Kay Bartholomew voor haar commentaar op hoofdstuk 2. 
Constante bronnen van sociale en wetenschappelijke steun waren de collega's bij de capgroep Gezondheidsvoorlichting, in het bijzonder secretaresse Marja Slangen, beheerder José Trottemant en systeembeheerders Jack Berben en Leon Kolenburg; de collega's bij het Centrum voor Gender en Diversiteit; de collega's van genderstudies gezondheidswetenschappen en geneeskunde; de collega's van de AIO-intervisiegroep Inez Bandell-Hoekstra, Anja van den Hout, Helga Nauta, Manon Savelkoul en Ellen Sebregts; de collega's van het bestuur van de Dutch Foundation for Women and Health Research; de collega's van de redactieraad van M/V Zorg; en enkele collega's van het vroegere Aletta, Landelijk centrum voor Vrouwengezondheidszorg en van TransAct, Landelijk expertisecentrum voor seksespecifieke zorg en seksueel geweld (Utrecht), in het bijzonder Maria van Bavel en Annemarie Wagemakers. Daarnaast heb ik veel geleerd van de studenten die ik heb begeleid bij hun jaarwerkstuk of scriptie: Linda Barentsen, Rianne Kasander, Tessa Reisser en Irina Tudor.

Verder had ik altijd een prettige dag als John Creusen me weer welkom heette in Debyeplein 1, als ik vrijwilligerswerk deed in de natuurtuinen Jekerdal van het Centrum voor Natuur en Milieu Educatie Maastricht Mergelland, of als ik gezellig kon bijpraten met mijn vrienden en mijn buren. Bijzonder gesteund en geïnspireerd voelde ik mij door mijn paranymphen Ineke Klinge en Greta Noordenbos; door mijn familie en in het bijzonder mijn ouders die er gewoon altijd voor me zijn; en door mijn partner Jeroen omdat hij Jeroen is. En dan was het altijd weer fijn als ik tussendoor op mijn motorfiets zat, die me al rijdend terugbracht naar de basis: mijn bekkenbodem.

Dianne 


\section{Curriculum vitae}

Dianne Alewijnse was born on November 25, 1971 in Eindhoven, The Netherlands. In 1990, she graduated from Gymnasium $\beta$ at the Stedelijke Scholengemeenschap in Maastricht. Between 1990 and 1996, she studied Biology at Utrecht University. She first specialised in the field of plant-ecofysiology and then followed through with her second specilisation in gender studies in biology and medicine. As a consequence, she became involved in the Dutch Women's Health Care movement. It was because of this interest in sex-specific health care and the possibility to include that particular point of view in a health education project, as well as her fascination with the symptom incontinence, that she became involved in the PhD-project that she has described in this thesis. 

